\title{
Dust devil sediment transport: From lab to field to global impact
}

\author{
Martina Klose • Bradley C. \\ Jemmett-Smith • Henrik Kahanpää · \\ Melinda Kahre - Peter Knippertz • \\ Mark T. Lemmon • Stephen R. Lewis • \\ Ralph D. Lorenz • Lynn D. V. Neakrase • \\ Claire Newman • Manish R. Patel • \\ Dennis Reiss - Aymeric Spiga - Patrick \\ L. Whelley
}

The final publication is available at http:// link. springer. com

\author{
M. Klose \\ Institute for Geophysics and Meteorology, University of Cologne, Cologne, Germany; \\ present address: USDA-ARS Jornada Experimental Range, Las Cruces, NM, USA \\ E-mail: mklose@nmsu.edu \\ B. C. Jemmett-Smith \\ Institute for Climate and Atmospheric Science, School of Earth and Environment, Univer- \\ sity of Leeds, Leeds, West Yorkshire, UK \\ E-mail: b.jemmett-smith@leeds.ac.uk \\ H. Kahanpää \\ Finnish Meteorological Institute, Helsinki, Finland \\ E-mail: henrik.kahanpaa@fmi.fi \\ M. Kahre \\ NASA Ames Research Center, Moffett Field, CA, USA \\ E-mail: melinda.a.kahre@nasa.gov \\ P. Knippertz \\ Institute of Meteorology and Climate Research, Karlsruhe Institute of Technology, Karl- \\ sruhe, Germany \\ E-mail: peter.knippertz@kit.edu \\ M. T. Lemmon \\ Department of Atmospheric Sciences, Texas A\&M University, College Station, TX, USA \\ E-mail: lemmon@tamu.edu
}


Abstract The impact of dust aerosols on the climate and environment of Earth and Mars is complex and forms a major area of research. A difficulty arises in estimating the contribution of small-scale dust devils to the total dust aerosol. This difficulty is due to uncertainties in the amount of dust lifted by individual dust devils, the frequency of dust devil occurrence, and the lack of statistical generality of individual experiments and observations. In this paper, we review results of observational, laboratory, and modeling studies and provide an overview of dust devil dust transport on various spatio-temporal scales as obtained with the different research approaches. Methods used for the investigation of dust devils on Earth and Mars vary. For example, while the use of imagery for the investigation of dust devil occurrence frequency is common practice for Mars, this is less so the case for Earth. Modeling approaches for Earth and Mars are similar in that they are based on the same underlying

S. R. Lewis

Department of Physical Sciences, The Open University, Milton Keynes, UK

E-mail: stephen.lewis@open.ac.uk

R. D. Lorenz

Johns Hopkins University Applied Physics Lab, Laurel, MD, USA

E-mail: Ralph.Lorenz@jhuapl.edu

L. D. V. Neakrase

Department of Astronomy, New Mexico State University, Las Cruces, NM, USA

E-mail: lneakras@nmsu.edu

C. Newman

Aeolis Research, Suite 205, 600 North Rosemead Boulevard, Pasadena, CA, USA, 91107

E-mail: claire@aeolisresearch.com

M. R. Patel

Department of Physical Sciences, The Open University, Milton Keynes, UK

E-mail: manish.patel@open.ac.uk

D. Reiss

Institut für Planetologie, Westfälische Wilhelms-Universität, Münster, Germany

E-mail: dennis.reiss@uni-muenster.de

A. Spiga

Laboratoire de Météorologie Dynamique, Université Pierre et Marie Curie, Paris, France

E-mail: spiga@lmd.jussieu.fr

P. L. Whelley

NASA Goddard Space Flight Center, Greenbelt, MD, USA

E-mail: patrick.l.whelley@nasa.gov 
theory, but they are applied in different ways. Insights into the benefits and limitations of each approach suggest potential future research focuses, which can further reduce the uncertainty associated with dust devil dust entrainment. The potential impacts of dust devils on the climates of Earth and Mars are discussed on the basis of the presented research results.

Keywords dust devils · dust emission · lab experiments · field measurements · modeling · dust environmental impact · sediment transport · Earth · Mars · planetary atmospheres

\section{Introduction}

Dust devils are a common occurrence on Mars and in semi-arid and arid regions on Earth when the surface is heated by insolation and convective turbulence is sufficiently developed (Chap. 5 [Rafkin et al., 2016]). The appearance of dust devils can be spectacular, but they are typically of small spatial extent (diameters of the order of magnitude $\sim 10^{0}-10^{1} \mathrm{~m}$ on Earth, $\sim 10^{1}-10^{2} \mathrm{~m}$ on Mars) and short duration $\left(\sim 10^{0}-10^{1} \mathrm{~min}\right)$, although exceptional larger and longer-lived cases have been reported (Balme and Greeley 2006; Greeley et al. 2010). The exact occurrence time and location of dust devils is hard to predict and makes in situ observations of dust devils a challenge. However, long-term monitoring by stationary instruments, as well as portable instruments that are transported into active dust devils, have provided valuable insights into dust devil characteristics on Earth (e.g. Sinclair 1969; Metzger et al. 2011; Lorenz et al. 2015). On Mars, cameras and meteorological in situ/remote sensing instrumentation on landers/orbiters have substantially advanced knowledge about Martian dust devils, thereby inspiring further research (e.g. Ryan and Lucich 1983; Thomas and Gierasch 1985; Schofield et al. 1997; Metzger et al. 1999; Cantor et al. 2006; Greeley et al. 2010; Ellehoj et al. 2010; Reiss et al. 2011; Moores et al. 2015). In addition, laboratory experiments and numerical modeling are powerful techniques to investigate dust devils (e.g. Neakrase et al. 2006; Kanak 2005; Gu et al. 2008; Ohno and Takemi 2010; Raasch and Franke 2011; Klose and Shao 2016). In all approaches, i.e. field, laboratory, and modeling, the estimate of total dust transport by dust devils and consequently the assessment of their environmental impact remain difficult due to the variety of dust devil sizes and intensities. Additionally, the dust devil dust load is 
ultimately dependent on the conditions of the land-surface in its path, which determines the abundance of soil dust particles available for entrainment.

The major impact of dust aerosols on the terrestrial and martian climates is through aerosol-radiation and aerosol-cloud interactions (e.g. Michelangeli et al. 1993; Rosenfeld et al. 2001; Bangert et al. 2012). Airborne dust particles scatter and absorb shortwave radiation and absorb and re-emit longwave radiation, leading to thermodynamic responses in the atmosphere (e.g. Sokolik and Toon 1996; Miller et al. 2014). Dust particles can also act as efficient ice nuclei both on Earth and Mars, thereby impacting cloud formation and cloud-climate feedback (e.g. Isono 1955; DeMott et al. 2003; Boucher et al. 2013). In the terrestrial environment, dust transport has further effects on ecosystems due to the transport of nutrients, minerals, and carbon, potentially acting as fertilizers (e.g. Bristow et al. 2010) or through deposition of pathogens or chemical contaminants (Shinn et al. 2000; Garrison et al. 2003). Human and animal health is influenced through inhalation of dust particles penetrating into the lung or carrying bacteria, fungi, pathogens, or allergens (Kellogg and Griffin 2006; Derbyshire 2007). Not least, reduced visibilities during dust events can cause severe traffic accidents.

Although the impact of dust aerosols on the global climate system has become a research focus in recent decades, the particular role and significance of dust devils compared to other meteorological dust injection processes is not clear. In this paper, we review estimates of dust transport in dust devils from in situ, remote sensing, laboratory, and modeling studies (Section 2). We summarize the occurrence frequency of dust devils on different spatial and temporal scales (Section 3) to provide a comprehensive picture of dust transported by dust devils on Earth and Mars (Section 4). The results are used to assess the impact of dust devils on climate and environment on local, regional, and global scales (Section 5). Findings for Earth and Mars are compared, indicating potential future directions for dust devil studies (Section 6).

\section{Dust transport in dust devils on Earth and Mars}

The mechanisms by which dust is entrained into vortices to form dust devils are still not fully understood. In general, three dust emission mechanisms are commonly referred to in aeolian research: saltation bombardment, aggregate 
disintegration, and aerodynamic entrainment (Shao, 2008). Saltation bombardment and aggregate disintegration are considered to be the most efficient dust emission mechanisms. Dust emission by both mechanisms is generated by saltation, the hopping motion of sand-sized particles or particle aggregates, causing localized impacts strong enough to inject dust particles into the atmosphere. Saltation is initiated as soon as the mean surface drag exceeds a threshold which depends on the surface conditions (Bagnold 1941; Greeley and Iversen 1985; Shao and Lu 2000). While this threshold is easily exceeded during dust storms, it remains controversial whether or not the drag in dust devils is sufficiently strong to initiate saltation. The aerodynamic entrainment of dust particles, i.e. dust uplift due directly to strong aerodynamic drag, has been neglected for a long time due to the on-average stronger inter-particle cohesive forces acting on dust particles compared to those acting on sand-sized particles. However, measurements show that inter-particle cohesion can vary over orders of magnitude even for particles of the same size, thereby allowing for stronger aerodynamic dust emission than previously thought (Zimon 1982; Klose et al. 2014; Shao and Klose 2016).

In addition to these three dust emission mechanisms, further mechanisms apply in the special case of dust devils. The small-scale vortices exhibit a substantial pressure drop in their center. The suction effect associated with this pressure drop may facilitate dust entrainment (Balme and Hagermann 2006). In a low-pressure environment such as on Mars, dust particles can also experience thermal lifting (Wurm et al. 2008; de Beule et al. 2014; Küpper and Wurm 2015). In the presence of a temperature gradient, thermal lifting is generated by thermal creep, a gas flow in the pores of a dust bed arising at pressures of few millibars. During an ongoing emission event, particle collisions can result in the development of an electrostatic field, which can reduce the entrainment threshold and enhance further emission (Kok and Renno 2006). A more detailed discussion of dust lifting mechanisms in dust devils is given in Chap. 10 (Neakrase et al., 2016).

This section describes different approaches (laboratory based, observational, and theoretical) to estimate the dust amount entrained by individual dust devils. Table 1 summarizes the values obtained by a range of such methods in several different studies for Earth and Mars. 
Table 1 Dust transport in individual dust devils

\begin{tabular}{|c|c|c|c|c|}
\hline \multicolumn{3}{|c|}{ Dust flux $\left[\mathrm{kg} \mathrm{m}^{-2} \mathrm{~s}^{-1}\right]$} & Method & Reference \\
\hline \multicolumn{5}{|c|}{ Earth } \\
\hline $2.2 \times 10^{-5}$ & - & $3 \times 10^{-3}$ & in situ & Gillette and Sinclair (1990) \\
\hline $1.4 \times 10^{-6}$ & - & $1.6 \times 10^{-4}$ & in situ (PM25) & Gillette and Sinclair (1990) \\
\hline $0.6 \times 10^{-3}$ & - & $4.4 \times 10^{-3}$ & in situ & Metzger (1999) \\
\hline $1 \times 10^{-4}$ & - & $1 \times 10^{-3}$ & in situ & Renno et al. (2004) \\
\hline $4.6 \times 10^{-6}$ & - & 100 & laboratory & Neakrase and Greeley (2010b) \\
\hline $4 \times 10^{-7}$ & - & $1.1 \times 10^{-4}$ & in situ (PM10) & Metzger et al. (2011) \\
\hline \multirow[t]{2}{*}{$1 \times 10^{-9}$} & - & $4 \times 10^{-7}$ & large-eddy simulation & Klose and Shao (2016) \\
\hline & \multicolumn{4}{|c|}{ Mars } \\
\hline $5 \times 10^{-4}$ & & & lander images & Metzger et al. (1999) \\
\hline $4 \times 10^{-9}$ & - & $1.6 \times 10^{-4}$ & lander images & Greeley et al. (2010) \\
\hline $2 \times 10^{-5}$ & - & 0.5 & laboratory & Neakrase and Greeley (2010b) \\
\hline $3.8 \times 10^{-7}$ & - & $1.2 \times 10^{-3}$ & orbital images & Reiss et al. (2014) \\
\hline
\end{tabular}

2.1 Laboratory experiments on dust transport in dust devils

A series of laboratory experiments were conducted between 2000 and 2009 at Arizona State University to try to understand fundamental controls on dust devil sediment lifting potential. The laboratory setup utilized the Arizona State University Vortex Generator (ASUVG), which consists of a $2.4 \times 2.4 \mathrm{~m}^{2}$ translatable table (both in the vertical and horizontal directions), below a cylinder assembly that houses the motorized fan that can be moved to different heights above the test surface controlling the diameter of the vortex on the test surface (Figure 1a) (Greeley et al. 2003; Neakrase et al. 2006; Neakrase and Greeley 2010b,a). The earliest experiments examined vortex threshold velocities for a range of particle sizes and densities both at terrestrial ambient and Mars-analog pressures (Greeley et al. 2003). Mars-analog atmospheric pressures ( $\sim 10 \mathrm{hPa}$, though with terrestrial ambient air composition) were attained using the Planetary Aeolian Laboratory at NASA Ames Research Center.

The initial ASUVG experiments showed that for dust-sized particles (diameters $<63 \mu \mathrm{m})$ the vortex threshold occurred at a lower value than the entrainment threshold in comparable boundary layer wind tunnel studies. This deviation from the standard boundary layer threshold suggests that sediment mobility under vortex conditions is subject to an additional lift component, 

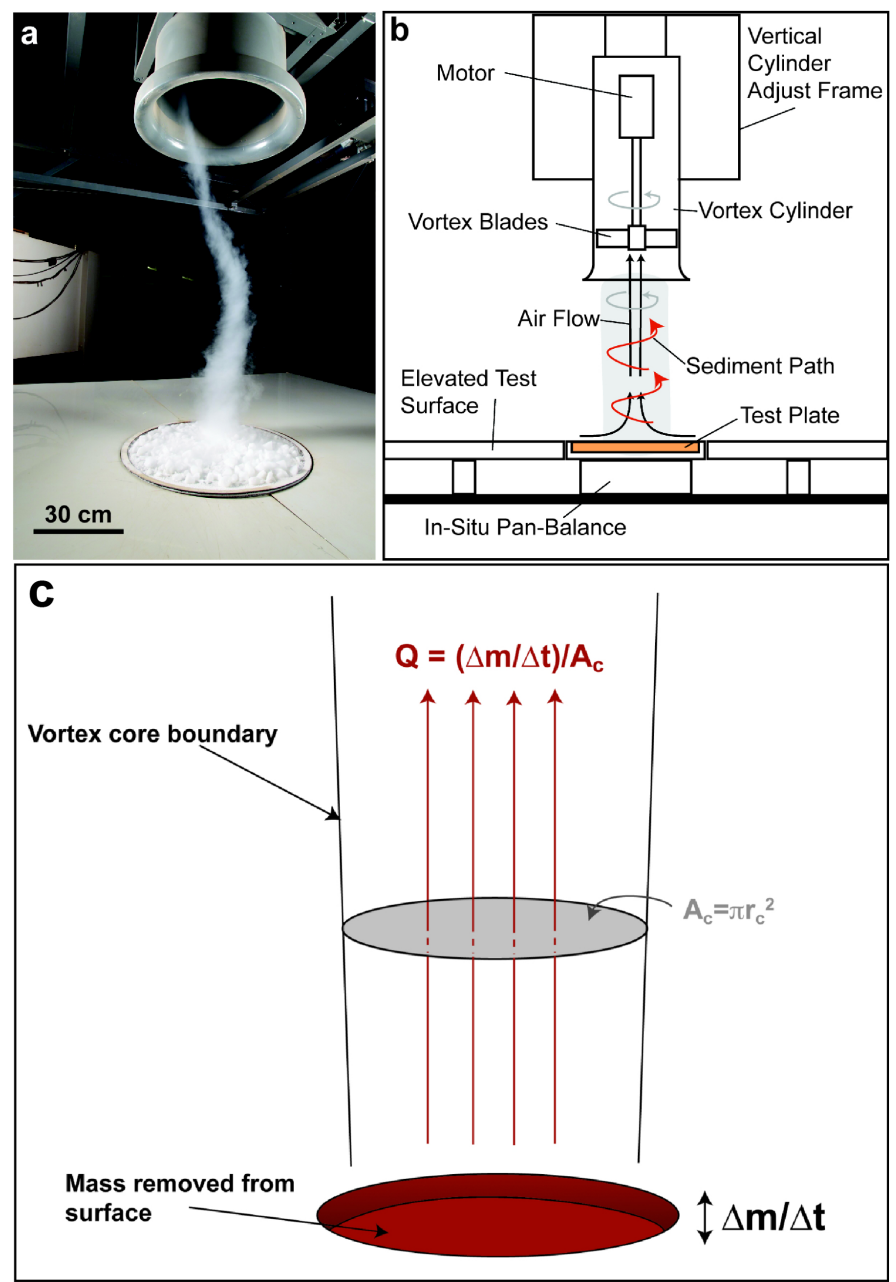

Fig. 1 Summary of Arizona State University Vortex Generator (ASUVG) sediment flux results. (a) Photo of the ASUVG; (b) Schematic of the ASUVG sediment flux experiments; (c) Cartoon of the mass calculation for vortex sediment flux; (Reprinted from Neakrase and Greeley 2010b, with permission from Elsevier).

which was dubbed the ' $\Delta p$-effect' by Greeley et al. (2003). This result implied that dust devils could be particularly efficient at lifting smaller particles (see also Chap. 10 [Neakrase et al. 2016]).

Building on the results of Greeley et al. (2003), sediment flux experiments were designed to investigate how dust devils lift sand and dust at terrestrial ambient and Mars-analog conditions using the ASUVG (Neakrase et al. 2006; Neakrase and Greeley 2010b). Using the results of the threshold experiments, 
the sediment flux study aimed to investigate the mass loss over time for conditions above threshold (Neakrase et al. 2006; Neakrase and Greeley 2010b). The sediment flux experiments consisted of an in situ pan balance beneath a $5 \mathrm{~mm}$ deep plate flush with the elevated test bed (Figure 1b). The plate was filled with different sediments and the ASUVG was set for different sized vortices (vortex diameter) and tangential velocities above the determined threshold. As the ASUVG was run for a set amount of time, mass measurements were made before and after each run to determine the change in mass per unit time $(\Delta m / \Delta t)$. Knowing the vortex parameters for each setup as determined by the height above the test surface and speed of the fan-blade assembly, the effective cross-sectional area of the vortex could be determined. Sediment flux was then determined to be

$$
Q=\frac{\Delta m}{\Delta t} \frac{1}{A_{c}}
$$

where $Q$ is the sediment flux through the area, $A_{c}$, of the vortex core (Figure 1c). Results from these experiments yielded empirical relationships for dust devil sediment flux as a function of a parameterized lift ratio $\left(\Delta p / u_{\theta}\right)$, represented by the average magnitude of the core pressure drop, $\Delta p$, and the maximum tangential velocity, $u_{\theta}$, as determined by curve-fitting the initial pressure data as a function of radius (Neakrase and Greeley 2010b). The sediment flux relationship as provided by Neakrase and Greeley (2010b) was

$$
Q \propto k\left(\frac{\Delta p}{u_{\theta}}\right)^{4}
$$

with $k$ being a parameter and was shown to be independent of ambient atmospheric pressure under this parameterization. This result averaged all sediment used in their experiments, which included both sand (particle diameter $\left.D_{p}>63 \mu \mathrm{m}\right)$ and dust $\left(D_{p} \leq 63 \mu \mathrm{m}\right)$. Average laboratory flux values were between $4.0 \times 10^{-6}$ and $100 \mathrm{~kg} \mathrm{~m}^{-2} \mathrm{~s}^{-1}$ for terrestrial ambient conditions and $2.0 \times 10^{-5}$ to $0.5 \mathrm{~kg} \mathrm{~m}^{-2} \mathrm{~s}^{-1}$ for martian analog conditions (Figure 2). Neakrase and Greeley (2010b) also investigated whether the individual curves for dust flux were lower than for sand flux. The results confirmed the initial threshold results by Greeley et al. (2003) in that dust particles are more effectively lifted in the presence of small $\Delta p$ than without, while more intensive $\Delta p$ have a stronger effect on sand-sized particles. This can likely be attributed to the larger tangential velocities in the latter case. 


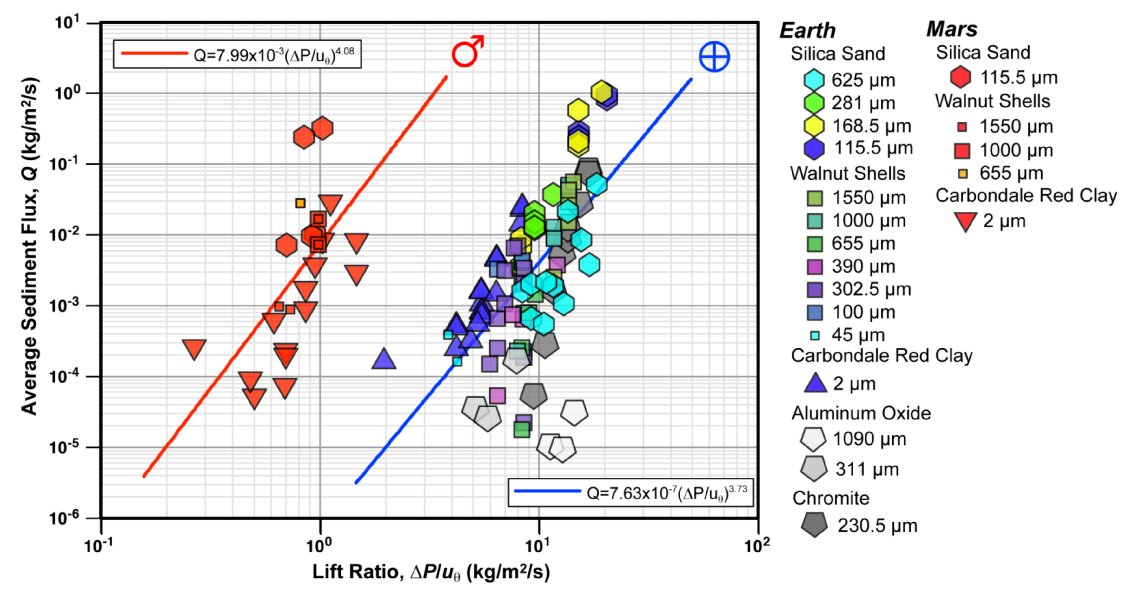

Fig. 2 Average sediment flux vs. lift ratio obtained for terrestrial ambient and Mars analog conditions for the entire tested range of sediments (Reprinted from Neakrase and Greeley 2010b, with permission from Elsevier).

Further experiments in sediment flux with the ASUVG investigated how vortex flow is affected by surface roughness elements. Neakrase and Greeley (2010a) used large roughness elements (i.e. sizes and spacing on the order of the size of the vortex core). Roughness is determined by the size and spacing of the nonerodible roughness elements and can be characterized by the roughness density, $\lambda$, also known as frontal-area index, defined by Lettau (1969) as

$$
\lambda=\frac{n b h}{S}
$$

where $n$ is the number of nonerodible roughness elements, $b$ is the element width, $h$ is the height of the element and $S$ is the total surface area over which the elements exist. Neakrase and Greeley (2010a) showed that for small roughness at this scale $(\lambda \approx 0.01)$ there seemed to be an optimal roughness, capable of enhancing vortex flow and, as a result, also the sediment flux. Neakrase and Greeley (2010a) suggested that this was a capability of the vortex of a given size and speed to adjust to the surface by conservation of angular momentum to fit between the roughness elements (enhancing flow) or expanding to incorporate the roughness elements (reducing flow). Both cases may be pertinent to the natural world where dust devils encounter large boulders or vegetation. Depending on the size and spacing of the roughness elements, dust devil sediment flux could be increased or decreased as a response to the vortex gaining or losing vorticity. 
Table 2 Calculated dust devil parameters including dust opacity from Mason et al. (2014)

\begin{tabular}{ccccc}
\hline Encounter & $r[\mathrm{~m}]$ & $z_{d d}[\mathrm{~m}]$ & $\tau_{a}$ at $450 \mathrm{~nm}$ & $\Delta p[\mathrm{hPa}]$ \\
\hline E1 & 11 & $>130$ & 0.8 & 0.30 \\
E2 & 4 & $>100$ & 0.6 & 0.42 \\
E3 & 5 & $>18$ & 0.2 & 0.29 \\
E4 & 7 & $>200$ & $2.3-2.7$ & - \\
E5 & 8 & $>200$ & $1.6-2.0$ & - \\
\hline
\end{tabular}

2.2 Field observations of dust devil dust transport - in situ measurements and remote sensing

Direct measurements of sediment fluxes within dust devils are difficult to obtain, hence most fluxes are calculated using measurements of dust concentrations and assumed or simultaneously obtained measurements of the vertical velocity within the dust devil. Sediment or dust fluxes are then calculated as vertical wind speed multiplied by particle concentration. It must be noted, however, that the so obtained fluxes are likely different to surface dust emission fluxes (compare Section 4.1). Gillette and Sinclair (1990) estimated particle fluxes based on in situ aircraft measurements of particle concentration and vertical wind speed in the updrafts of several dust devils at altitudes of 142 and $330 \mathrm{~m}$. Although the corresponding method paper was never published, Gillette and Sinclair (1990) summarized mean dust flux estimates for different dust devil sizes ranging from (a) $2 \times 10^{-5} \mathrm{~kg} \mathrm{~m}^{-2} \mathrm{~s}^{-1}$ for dust devils $<3 \mathrm{~m}$ in diameter to (b) $3 \times 10^{-3} \mathrm{~kg} \mathrm{~m}^{-2} \mathrm{~s}^{-1}$ for dust devils $>30 \mathrm{~m}$ in diameter. However, the contribution of finer particles $(<25 \mu \mathrm{m}$ diameter $)$ to these total dust flux estimates ranged only from $1.4 \times 10^{-6}$ to $1.6 \times 10^{-4} \mathrm{~kg} \mathrm{~m}^{-2} \mathrm{~s}^{-1}$ for (a) and (b), respectively. For dust devils of all sizes, Gillette and Sinclair (1990) obtain a total dust flux $(<25 \mu \mathrm{m}$ diameter $)$ of $2.8 \times 10^{-4} \mathrm{~kg} \mathrm{~m}^{-2} \mathrm{~s}^{-1}$ for their test area in the southwestern US. Renno et al. (2004) estimated a particle flux of $1 \times 10^{-3} \mathrm{~kg} \mathrm{~m}^{-2} \mathrm{~s}^{-1}$ based on LIDAR measurements of particle concentration in one dust devil at $100 \mathrm{~m}$ height and using the peak vertical wind speed of $10 \mathrm{~m} \mathrm{~s}^{-1}$ measured in the dust devil using a sonic anemometer. The most reliable terrestrial in situ measurements were made by Metzger (1999) and Metzger et al. (2011). Metzger (1999) measured sediment loads of total suspended particles (TSP) in dust devils and calculated sediment fluxes 
in the range of $0.6 \times 10^{-3}-4.4 \times 10^{-3} \mathrm{~kg} \mathrm{~m}^{-2} \mathrm{~s}^{-1}$ for five dust devils near the surface using simultaneously obtained measurements of vertical speeds within the dust devils. Near-surface measurements of twelve dust devils by Metzger et al. (2011) gave suspended particle loads of particulate matter $<10 \mu \mathrm{m}$ in diameter (PM10). The authors calculated maximum dust fluxes ranging from $4 \times 10^{-7}-1.1 \times 10^{-4} \mathrm{~kg} \mathrm{~m}^{-2} \mathrm{~s}^{-1}$ using again simultaneously obtained measurements of vertical speeds within the dust devils. The authors found that the mean flux in dust devils is about one third of the measured maximum flux.

Mason et al. (2014) performed a field study on dust devils in 2009 in the Southern Nevada Desert (Eldorado Valley, USA) in a closed playa basin, using a chase vehicle-encounter approach in which instruments were driven into existing dust devils. Results were presented for 5 well-defined dust devil encounters, E1 - E5. It should be noted that the chase-encounter approach is a targeted approach to observation, and thus introduces a selection effect into the results, biased towards larger, more defined dust devils that are more easily tracked. By using optical instrumentation fixed to the top of the vehicle, and ensuring that the dust devil trajectory passes over the instrumentation, the dust loading of the dust devils was recorded. For the encounters E1 - E5, the radius of the dust devil $(r)$, dust devil height $\left(z_{d d}\right)$, apparent dust optical depth $\left(\tau_{a}\right)$ and pressure drop $(\Delta p)$ were defined, shown in Table 2 . The physical dimensions of the dust devils were calculated from images containing known reference lengths. The high dust loading and size of encounters E4 and E5 led to an undefined vortex core, and therefore, a range of $\tau_{a}$ is specified.

Table 2 shows five encounters that were studied in detail for dust optical property retrieval, however a larger data set of encounters was recorded for investigating the relation between $\Delta p$ and dust loading. Greeley et al. (2003) and Neakrase et al. (2006) suggested that vortices are more efficient at dust lifting than non-rotating boundary layer flows due to the pressure deficit in the vortex centers. This would indicate that encounters with more intense pressure drops may have higher dust loadings. The extinction coefficient $k_{\text {ext }}$ (attenuation per unit length) was calculated for 23 dust devils using $\tau_{a}$ and $r$ and plotted against $\Delta p$, shown in Figure 3. There is no unique relationship between dust loading and $\Delta p$ as the dust load depends not only on entrainment threshold, but also on the dust devil dimensions and shape, tilt, and in a natural environment on dust supply. It is clear, however, that as $\Delta p$ increases 


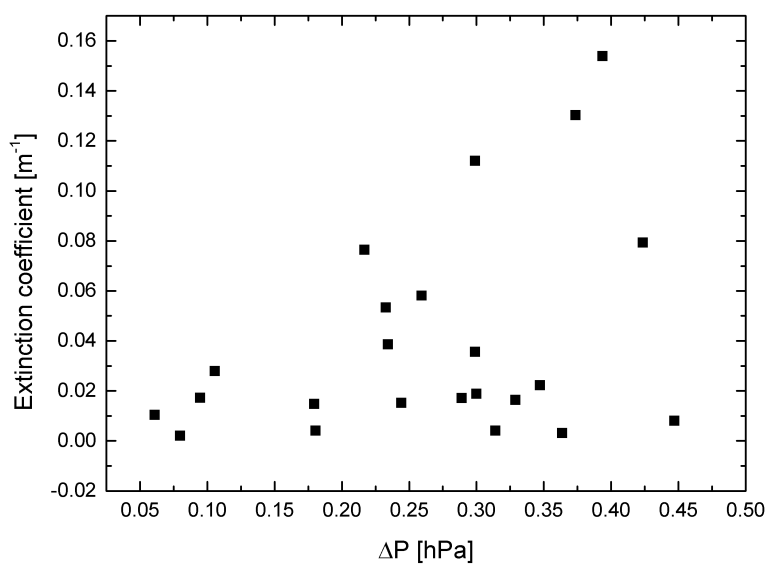

Fig. 3 Extinction coefficient $\left[\mathrm{m}^{-1}\right]$ (representing dust loading) as a function of $\Delta p[\mathrm{hPa}]$ for 23 dust devil encounters (modified from Mason et al. 2014).

so does the maximum dust loading (and thus the range of dust loadings) that can be produced.

Lorenz and Jackson (2015) observed dust devil activity in summer 2013 at four spots in the same Eldorado field site as studied by Mason et al. (2014). Lorenz and Jackson (2015) used data loggers that recorded pressure and incident sunlight at a high cadence for over a month. Direct encounters and near-misses of boundary layer vortices are indicated by a sharp drop in pressure: when these vortices are dust-laden, there may also be a drop in incident sunlight. Not all dust devil encounters lead to such a drop - if the dust devil passes in the anti-sun direction (e.g. north or east during the afternoon), the dust column may not block the line-of-sight to the sun. Nonetheless, this measurement approach gave a census of dust associated with dust devil activity. Of $50-80$ pressure dips $(>0.3 \mathrm{hPa})$ detected per 100 station-days at each of four locations, about $40 \%$ had no detectable solar attenuation. Some fraction of these were simply anti-sun misses, but some are doubtless close encounters with devils with undetectably small amounts of dust. About $40 \%$ of the total had obscuration of $1 \%$ or more (indicating a line-of-sight opacity of $\sim 0.01$ ) and about $10 \%$ had $5 \%$ obscuration or more. While large pressure drops (indicating close distances and/or intense vortices) could have large or small obscuration, it was noticed that large obscuration was only found with the more intense 
pressure drops: the scatter of attenuation $A[\%]$ against $\Delta p[\mathrm{hPa}]$ points appeared to have an upper limit of $A \approx 50 \Delta p$. This exact relationship may be site-dependent, however; indeed, differences in the populations between the four stations, separated by only $\sim 1 \mathrm{~km}$, were noted, making evident the significance of small-scale variations that are not practically captured in numerical models except in a statistical manner.

Metzger et al. (1999) measured dust column opacities for a dust devil observed by the lander camera at the Mars Pathfinder landing site and used a terrestrial vertical velocity estimate of $7 \mathrm{~m} \mathrm{~s}^{-1}$, calculating a vertical flux of $5 \times 10^{-4} \mathrm{~kg} \mathrm{~m}^{-2} \mathrm{~s}^{-1}$. Reiss et al. (2014) measured dust column opacities for three dust devils using orbiter images and calculated their dust loads. Minimum and maximum calculated dust fluxes were in the range between $3.8 \times 10^{-7}$ and $1.2 \times 10^{-3} \mathrm{~kg} \mathrm{~m}^{-2} \mathrm{~s}^{-1}$ assuming vertical velocity ranges between 0.1 and $10 \mathrm{~m} \mathrm{~s}^{-1}$.

From the surface of Mars, statistically significant datasets of dust devil observations have been limited to imaging campaigns from the Mars Exploration Rover Spirit (Greeley et al. 2006, 2010). Over three martian years the cameras onboard Spirit routinely imaged dust devils on the floor of Gusev crater (Figure 4). Greeley et al. (2010) determined the density of dust within vortices detected by Spirit by deriving atmospheric opacities from the images and comparing values obtained within the dust devils against background values. The obtained dust concentrations varied from $2.1 \times 10^{-9}-2.5 \times 10^{-4} \mathrm{~kg} \mathrm{~m}^{-3}$. Further, vertical wind speeds inside vortex cores were determined by identifying features such as apparent "clots" of dust within the dust devils and tracking them in sequential images. The result was that the distribution of vertical speeds ranged from 0.04 to $17.0 \mathrm{~m} \mathrm{~s}^{-1}$. From these observations Greeley et al. (2010) calculated that the vertical dust flux for individual vortices ranged from $4.0 \times 10^{-9}-1.6 \times 10^{-4} \mathrm{~kg} \mathrm{~m}^{-2} \mathrm{~s}^{-1}$.

Unfortunately there was no meteorological instrumentation onboard the Spirit rover. However, three other Mars landers have observed dust devils by both imaging and meteorological measurements. These landers are Mars Pathfinder (Murphy and Nelli 2002; Ferri et al. 2003), Phoenix (Ellehoj et al. 2010) and MSL Curiosity (Moores et al. 2015; Kahanpää et al. 2016; Steakley and Murphy 2016). Pathfinder and Phoenix operated for only a few months and took a small number of images suitable for a dust devil survey. Thus only 


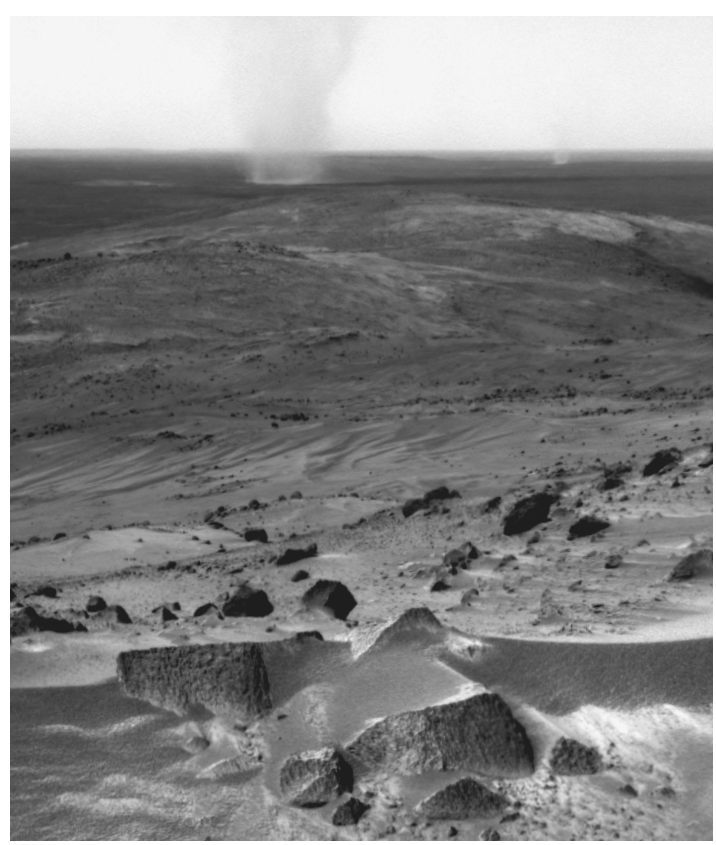

Fig. 4 Dust devil in Gusev: A navigation camera image of a dust devil in Gusev crater. Taken on Mars Exploration Rover Spirit on 22 August 2004 mission day (sol) 581 (Product ID: 2N 177950967 RAD AD ND P0645 L0 C1).

14 and 37 dust devils were identified from the images taken by these landers, respectively (Ferri et al. 2003; Ellehoj et al. 2010). Curiosity has operated to date for more than one martian year, but landed on an area where the dust devil activity is low and was thus able to detect only one plausible dust devil during its first Earth year on Mars (first 360 sols), despite an intensive imaging campaign of "dust devil search movies" (Moores et al. 2015). No more dust devils have been detected between sols 361 and 681 (J. Moores, 2016, personal communication). All of these three landers were equipped with high-resolution pressure sensors and detected numerous transient pressure drops apparently caused by convective vortices. A distinctive feature of these pressure drops is that they are small: less than $4.8 \mathrm{~Pa}, 3.6 \mathrm{~Pa}$, and 3.0 $\mathrm{Pa}$ for Pathfinder, Phoenix, and Curiosity (first 681 sols), respectively (Murphy and Nelli 2002; Ellehoj et al. 2010; Kahanpää et al. 2016; Steakley and Murphy 2016).

Moores et al. (2015) compared the statistics of dust devil detections in the image data and pressure data of Pathfinder. A prediction of the number of dust devils that should be visible in the images was calculated based on the 
assumption that all identified pressure drops with magnitude greater than a preset threshold, $\Delta p_{t}$, were caused by dust-lifting vortices. The result was that with $\Delta p_{t}=2.0 \mathrm{~Pa}$ the number of visually detected dust devils matched the prediction. This is only a rough estimate given that it is based on a statistically small number of both pressure drops and dust devils detected by Pathfinder. Also, the detected pressure drops are lower than the central pressure drops of the vortices as they do not pass right over the sensor, so this result should be taken as a lower limit of the central pressure drops occurring in Martian dust devils. However, this calculation indicates that it is improbable that all dust devils seen by Pathfinder were caused by vortices much stronger than what was detected in the pressure data.

Assuming that a dust devil is in cyclostrophic balance, the tangential wind speed, $u_{\theta}$, is proportional to the square root of the central pressure drop, $\Delta p$ (Sinclair 1973; Renno et al. 2000). By making use of the ideal gas law, $u_{\theta}$ can be expressed as

$$
u_{\theta}=\sqrt{R T \frac{\Delta p}{p_{s}}}
$$

where $R$ is the specific gas constant, $T$ is air temperature, and $p_{s}$ is surface pressure. The magnitudes of the largest pressure drops detected by Pathfinder, Phoenix and Curiosity can be transferred into tangential wind speeds using this relationship. The results are 17, 14 and $13 \mathrm{~m} \mathrm{~s}^{-1}$ for Pathfinder, Phoenix and Curiosity (first 681 sols), respectively. These values can be compared to threshold wind speeds for dust lifting by vortices at martian pressure, determined in laboratory experiments (Section 2.1). Greeley et al. (2003) concluded that the threshold for fine dust $(2 \mu \mathrm{m})$ was $\sim 20-30 \mathrm{~m} \mathrm{~s}^{-1}$, and Neakrase and Greeley (2010b) found that some fine dust was lifted already at $18 \mathrm{~m} \mathrm{~s}^{-1}$. Taking into account (a) that the central pressure drops of the vortices will generally be deeper than the detected pressure drops, unless the vortices pass directly over the sensor, (b) that the translational motion of the vortices also affects their ability to lift dust, and (c) the lower gravity of Mars, the tangential wind speeds calculated for the strongest vortices detected by Pathfinder agree with the minimum requirements for dust lifting as determined in the laboratory. The non-detection of practically any dust devils during Curiosity's first 681 sols, despite plenty of dust to lift, suggests that $3.0 \mathrm{~Pa}$ or $13 \mathrm{~m} \mathrm{~s}^{-1}$ is a lower limit for the dust lifting threshold on Mars. This limit is roughly in agreement 
with the threshold of $\Delta p_{t}=2.0 \mathrm{~Pa}$ obtained by Moores et al. (2015), which was based on comparing the statistics of vortex detections in the image and pressure data measured by Pathfinder.

Choi and Dundas (2011) used images taken from orbit to study the wind fields inside martian dust devils. They reported wind velocities within four dust devils imaged by the High Resolution Imaging Science Experiment (HiRISE) onboard Mars Reconnaissance Orbiter (MRO). HiRISE images the surface through three color filters with $\sim 0.1 \mathrm{~s}$ intervals. Wind velocities were derived by automated tracking of dust devil cloud contrast features between the frames imaged through the different color filters. The determined composite wind velocities, i.e. the sum of translational and tangential wind, had typically magnitudes between 20 and $30 \mathrm{~m} \mathrm{~s}^{-1}$. When assuming cyclostrophic balance, the detected tangential wind speed profiles corresponded to $0.25-1 \%$ pressure decreases in the dust devil cores relative to ambient pressure. This agrees with the strongest pressure drops detected by Pathfinder and Phoenix.

2.3 Numerical modeling of dust devils and associated dust transport large-eddy simulation

Large-eddy simulation (LES) is a term used to describe the numerical simulation of atmospheric flow at horizontal grid spacings of $\sim 10^{0}-10^{2} \mathrm{~m}$ (Deardorff 1970). At such high spatial and associated high temporal resolution, most turbulence structures are resolved, making LES a powerful tool to study turbulent phenomena (see Chapt. 7 [Spiga et al., 2016] for more details on LES). To date, most LES studies related to terrestrial dust devils have focused on vortex formation conditions and/or their physical characteristics (Kanak et al. 2000; Kanak 2005; Zhao et al. 2004; Gu et al. 2008; Ito et al. 2010b; Ohno and Takemi 2010; Raasch and Franke 2011; Klose and Shao 2013), with only a few such studies examining the fluxes of sand or dust.

Zhao et al. (2004) injected sand particles into their terrestrial LES to study particle trajectories in a dust devil. The authors found that particles with diameters up to $160 \mu \mathrm{m}$ could be transported in their simulated dust devils, with the largest particles being carried in the periphery of the vortices. Ito et al. (2010a) included the empirical formulation for dust lifting of Loosmore and Hunt (2000) into their LES and studied dust suspension in a convective plane- 

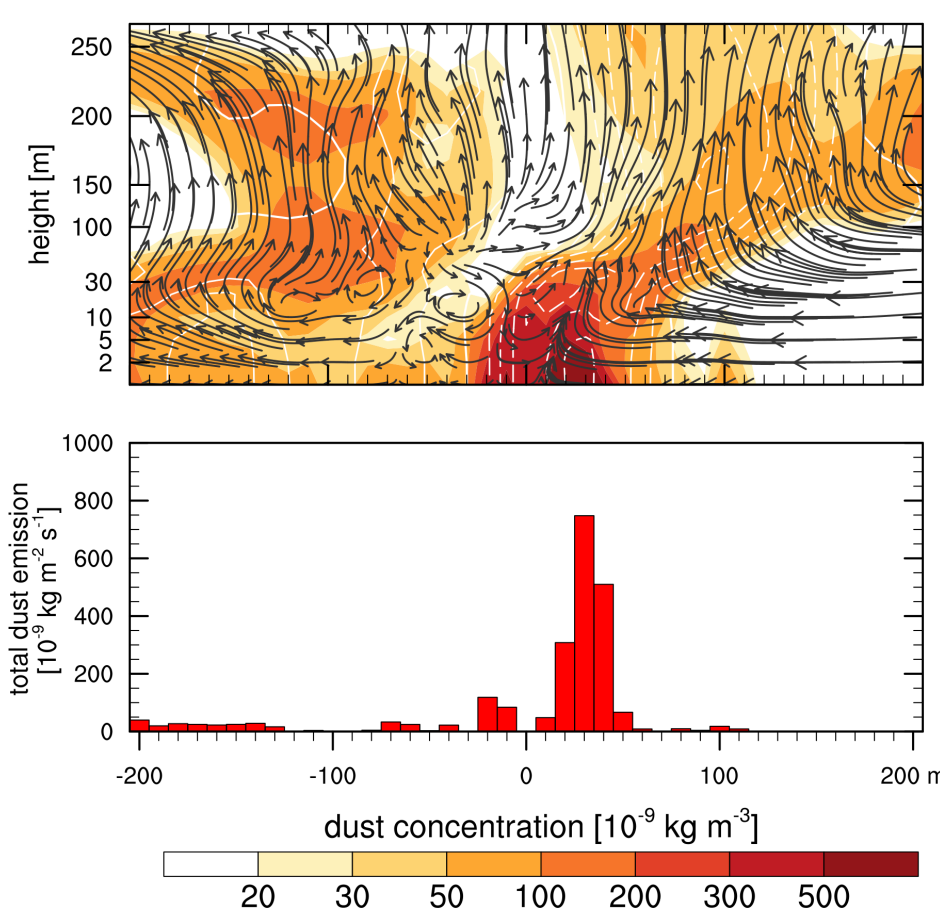

Fig. 5 (Top) Vertical cross section of dust concentration (shaded), perturbation pressure (contour lines), and turbulent wind vectors through a dust devil identified in LES; (Bottom) linear cross section of dust emission corresponding to the top plot. (Reprinted from Klose and Shao 2016, with permission from Elsevier).

tary boundary layer (PBL) on Earth. Loosmore and Hunt (2000) had measured dust flux in a wind tunnel for wind speeds below the threshold for saltation, but without particular focus on convective turbulence. They related dust emission flux to friction velocity only and therefore the dust fluxes simulated by Ito et al. (2010a) for a convective boundary layer at low mean wind speed (and thus on average small friction velocity) are relatively low $\left(10^{-8} \mathrm{~kg} \mathrm{~m}^{-2} \mathrm{~s}^{-1}\right)$. Klose and Shao (2013) coupled their terrestrial LES with a size-resolved dust emission scheme representing the aerodynamic entrainment of dust particles by atmospheric turbulence (Klose and Shao 2012) and also employed parameterizations for dust transport and deposition. The dust transport by dust devils was thus explicitly simulated. Although turbulent dust emission is generally weak and of the orders of magnitude $10^{-9}-10^{-7} \mathrm{~kg} \mathrm{~m}^{-2} \mathrm{~s}^{-1}$, Klose and Shao (2016) showed in a follow-up work, using an upgraded version of their scheme which was calibrated against field measurements of convective dust 
emission (Klose et al. 2014), that turbulent dust emissions can reach an order of magnitude $10^{-6} \mathrm{~kg} \mathrm{~m}^{-2} \mathrm{~s}^{-1}$ under favorable conditions such as in dust devils or dust plumes. Figure 5 shows a linear cross-section of dust emission through an individual dust devil detected in an LES run together with the corresponding vertical cross-section of PM20 dust concentration, perturbation pressure, and turbulent wind vectors. Dust emission reached values of up to $8 \times 10^{-7} \mathrm{~kg} \mathrm{~m}^{-2} \mathrm{~s}^{-1}$ and the maximum dust concentration in this dust devil was $\sim 5 \times 10^{-7} \mathrm{~kg} \mathrm{~m}^{-3}$. The dust emission in dust devils varied strongly from case to case but the maximum emissions varied only to an upper-limit envelope, which was related to atmospheric stability by Klose and Shao (2016). The finding is consistent with observations of solar attenuation in dust devils, which indicated that dust devil intensity does not uniquely determine the dust load in individual dust devils, but that the maximum possible dust load increases with dust devil intensity (Mason et al. 2014; Lorenz and Jackson 2015, see Section 2.2).

Assessing dust devil particle fluxes using LES for Mars, both for individual dust devils and for an area prone to dust devil occurrence, has proven difficult. The main difficulty is that the current martian literature focuses more on convective vortices than on actual dust devils - the subset of convective vortices capable of lifting dust (see Chap. 7 [Spiga et al., 2016] and references therein). However, vortices strong enough to potentially lift dust and become dust devils (and LES tends to show this could be as low as a few percent of the actual vortices arising from PBL convection) show characteristics similar to those observed for dust devils by landers and orbiters. These characteristics include: pressure drops of a few Pascals, sizes from a few tens to hundreds of meters, vertical extension of about a kilometer, and durations from a few tens to at most a few thousands of seconds (Rafkin et al. 2001; Toigo and Richardson 2003; Michaels and Rafkin 2004; Spiga and Forget 2009; Gheynani and Taylor 2011). Those parameters are generally all larger for martian dust devils than for their terrestrial counterparts, which is consistent with the generally larger dust flux supposedly originating from Mars's dust devils compared to Earth's.

Michaels (2006) was the first to include a saltation bombardment scheme in a Mars LES, calculating bulk dust fluxes partitioned according to a predefined particle size distribution. While Michaels focused on the formation of dust devil tracks and did not aim to calculate dust fluxes, his simulation showed that the 
modeled dust devil track reached a maximum depth of about $8 \mu \mathrm{m}$ only, with the majority of the track being less than $1.5 \mu \mathrm{m}$ deep. This contrast results from "pulses " in vortex intensity, likely caused by the fluctuations of convective cells within which the vortex is embedded. Michaels (2006) noted that particle redeposition is prominent for the largest particles (about $100 \mu \mathrm{m}$ radius) but negligible for particles smaller than $10 \mu \mathrm{m}$. Overall, the net redeposition of dust was very small anywhere in the model domain while the dust devil was active, and was slightly larger after its cessation. The LES results demonstrate that a dust devil should be able to transport dust particles rapidly from the surface to a height of several kilometers. Along the majority of its track, the modeled vortex exhibited a central pressure drop of $\sim 4.5 \mathrm{~Pa}$, which compares well with the strongest vortices observed by Mars Pathfinder (Murphy and Nelli 2002). However, dust lifting in the vortex was probably even stronger than could be expected for such a pressure drop for two reasons: (1) the pulses in vortex intensity led to a pressure drop which could temporarily rise up to $6.5 \mathrm{~Pa}$; and (2) the vortex was not symmetrical and not completely in cyclostrophic balance so that the vortex wind speed sometimes exceeded $19 \mathrm{~m} \mathrm{~s}^{-1}$ at certain locations.

Despite the success in reproducing dust-devil like vortices, LES models still need further improvement before they can be used to assess dust fluxes generated by dust devils. As a result, authors were cautious to present dust flux estimates in the existing martian LES literature. Several difficulties remain, such as LES models being highly idealized and lacking the influence of e.g. rugosity caused by small-scale topography or small-scale albedo contrasts. The parameterization of dust lifting by resolved turbulent winds on Mars is also prone to many shortcomings - including the major paucity in measurements on Mars to validate models derived from terrestrial measurements. Another major complication is a lack of knowledge of the availability of dust in a given area, which might explain the pronounced contrasts of observed dust devil activity between various regions on Mars, while LES modeling, along with insitu pressure measurements, indicates that the formation of convective vortices is widespread. 


\section{Dust devil occurrence frequency on Earth and Mars}

To assess the relevance of dust devils, it is necessary to estimate their occurrence frequency. Dust devil occurrence varies considerably on different spatiotemporal scales and in different regions on Earth and Mars. Three approaches are examined in this section. Dust devil occurrence frequency is evaluated based on: (i) their formation conditions - i.e., the local meteorology and surface characteristics (Section 3.1; see also Chap. 5 [Rafkin et al., 2016]); (ii) statistical analysis of observations such as dust devil tracks (Section 3.2, see also Chap. 4 [Reiss et al., 2016]); and (iii) regional and global model results (Section 3.3).

A summary of dust devil occurrence densities as identified from visual surveys on Earth and Mars, augmented by results from modeling studies, is shown in Figure 6. The largest densities of vortices detected in LES and inferred from field pressure measurements correspond to a couple of thousand vortices per $\mathrm{km}^{2}$ per day. Most of these are too weak to lift dust, and the largest densities of observed dust devils (from the surveys observing the smallest area and thus efficiently detecting the most abundant small devils) are of the order of $100 \mathrm{~km}^{-2}$ day $^{-1}$. Other visual surveys on both Earth and Mars yield densities that fall off with survey area $A$ roughly as $1 / A$, since only scarce large devils are seen in wide area surveys. For more details on dust devil populations from the perspectives of modeling, remote sensing, and in situ observations, see Chap. 8 (Lorenz and Jackson, 2016).

3.1 Dust devil occurrence frequency based on meteorological and land-surface conditions

The controls on the dust devil frequency of occurrence on Earth and Mars can broadly be determined by the availability of sand, dust or debris, the complexity of the terrain, and the meteorological conditions that prevail. The general characteristics for dust devil formation on Earth are summarized by the existence of the following conditions: (1) intense surface heating through insolation and a strong superadiabatic atmospheric temperature lapse rate, which implies a low soil moisture and a very high Bowen ratio (ratio of sensible to latent heat flux); (2) relatively weak ambient winds, i.e. generally less than $10 \mathrm{~m} \mathrm{~s}^{-1} ;(3)$ smooth arid terrain with some rock cover but few trees, buildings, 


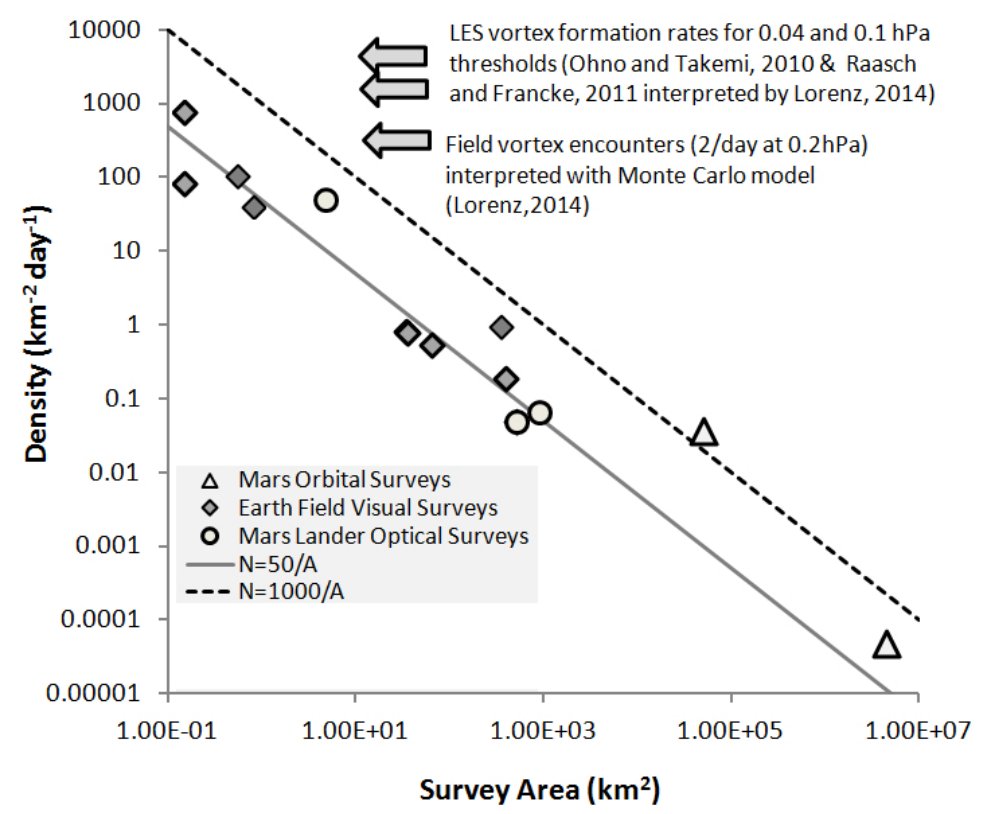

Fig. 6 A compilation of observed dust devil frequencies from visual surveys on Earth and Mars, derived from Lorenz (2013). There is a systematic variation of observed counts with area surveyed, presumably because large area surveys (especially those from orbit - triangles) only detect the larger, rarer dust devils. Lines show linear relationships between dust devil density and survey area, A, as a reference. Also indicated is the vortex generation rate of $300 \mathrm{~km}^{-2}$ day $^{-1}$ required, with assumptions on longevity and advection speed, to reproduce in a Monte Carlo model (Lorenz 2014) the observed pressure dips above $20 \mathrm{~Pa}$ seen in terrestrial field data to indicate vortices (not all of which may be dust-laden). The vortex formation rate measured in two different terrestrial LES simulations, of $4500 \mathrm{~km}^{-2} \mathrm{day}^{-1}$ and $1500 \mathrm{~km}^{-2} \mathrm{day}^{-1}$ for vortices with core pressure drops of more than $4 \mathrm{~Pa}$ and $10 \mathrm{~Pa}$ and vorticities of more than $1 \mathrm{~s}^{-1}$ and $0.15 \mathrm{~s}^{-1}$, respectively, are also shown. Most of these rather weak vortices will be dustless, however. The Mars and Earth rates appear, with survey area taken into account, to differ only by a factor of a few at most.

or grassy areas; (4) relatively level to gently sloping topography (Balme and Greeley 2006; Oke et al. 2007b; Kurgansky et al. 2011). See Chap. 5 (Rafkin et al., 2016) for a detailed discussion of dust devil formation conditions.

Dust devil activity is known to vary within seasonal, daily, and episodic cycles and across terrain types (Sinclair 1969; Oke et al. 2007b). On Earth, dust devils have a diurnal cycle that closely resembles the most active period of the convective boundary layer cycle, with activity generally observed between 10:00 - 17:30 local time (Balme and Greeley 2006). Until recently no global 
(a)

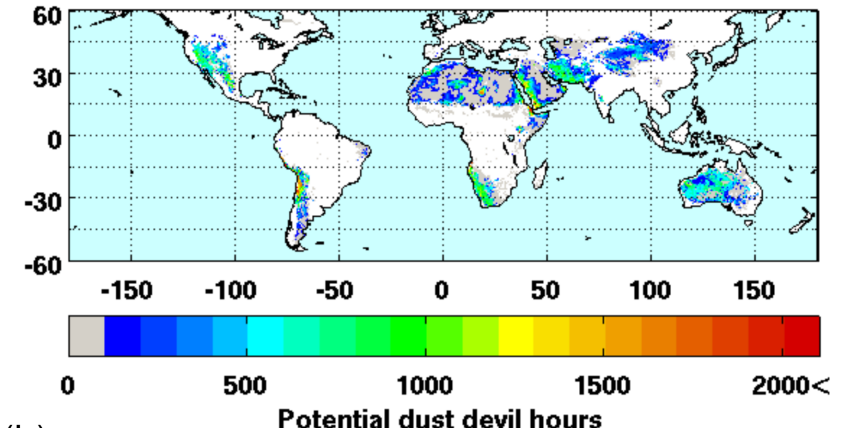

(b)
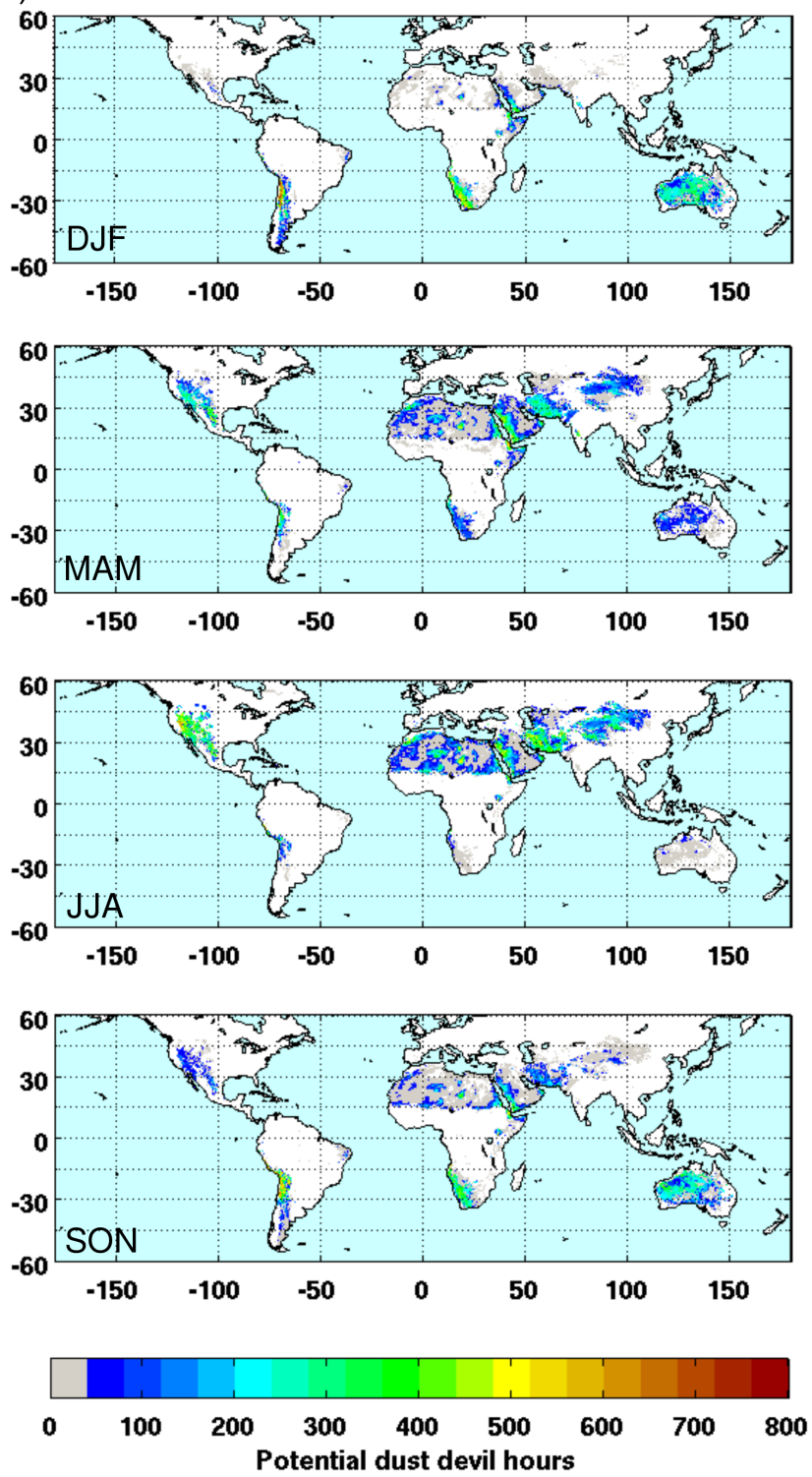

Fig. 7 Climatology of potential dust devil hours (PDDP ${ }_{\text {hours }}$; mean of 2012 and 2013) using criteria of $w_{*} / u_{*}>5$ and near-surface lapse rate $>8.5 \mathrm{~K} \mathrm{~m}^{-1}$ from respectively Lyons et al. (2008) and Ansmann et al. (2009) for (a) annual total, and (b) seasonal totals (modified from Jemmett-Smith et al. 2015). 
quantitative estimate of dust devil occurrence frequency had been conducted. The study by Jemmett-Smith et al. (2015) was the first to quantify regional and global potential of dust devil occurrence frequency on Earth (where the actual occurrence depends on the availability of dust), with results shown to be broadly consistent with observations (see Balme and Greeley 2006). This was achieved by using meteorological constraints to identify potential dust devil hours from global model outputs (operational analyses from the European Centre for Medium-Range Weather Forecasts, ECMWF; analyses data are model data with assimilation of observational data to provide the most realistic representation of a given time), with $\sim 20 \mathrm{~km}$ horizontal resolution and hourly temporal resolution. The relatively high spatio-temporal resolution made it possible to sufficiently resolve the diurnal cycle as well as the conditions required for dust devils to exist, which was not possible before. Grid-box potential dust devil active hours were defined as hours when thresholds of $w_{*} / u_{*}$ (ratio of convective velocity scale, $w_{*}$, and friction velocity, $u_{*}$; or equivalently convective boundary layer height over Obukhov length, $-h / L$, as used by Deardorff (1978); Hess and Spillane (1990); Kurgansky et al. (2011)) and near-surface temperature lapse rate (Ryan 1972; Oke et al. 2007b; Ansmann et al. 2009) were exceeded; in this case the $8.5 \mathrm{~K} \mathrm{~m}^{-1}$ lapse rate criterion from Ansmann et al. (2009) and the $w_{*} / u_{*}>5$ criterion from Lyons et al. (2008) were used. The combination of these two criteria provided a framework that incorporates all known measures of local meteorology that determine when and where dust devils are likely to occur, providing a dust source is available. Measures of atmospheric stability are also used for dust devil dust lifting parameterizations in martian global models (see Sections 3.3.2 and 4.2.2).

Results show a diurnal cycle as well as geographical, seasonal and annual variations in dust devil frequency of occurrence, with a clear indication that arid areas, in both hemispheres, have the highest potential dust devil activity (Figure 7). As expected, minima occur in winter and maxima in summer, showing a clear latitudinal dependence (related to solar insolation) with low latitude regions (generally between $10^{\circ}$ and $30^{\circ}$ North and South) experiencing higher total values and smaller seasonal variations. The total area of potential dust devil activity within a year is $\sim 3.7 \times 10^{7} \mathrm{~km}^{2}$, with a mean active period of $\sim 205 \mathrm{~h} \mathrm{yr}^{-1}$ (Figure $7 \mathrm{a}$ ). Potential occurrence tends to be restricted to hot spot regions, with dust devil active hours on the order of $2500 \mathrm{hyr}^{-1}$ 
(equivalent to $\sim 7 \mathrm{~h}$ of activity on average per day); an indication that these regions are active year round (Jemmett-Smith et al. 2015). Notable hot spots include parts of the Atacama and Sechura deserts of South America, which are known dust devil breeding grounds (see Kurgansky et al. 2011; Hesse 2012; Reiss et al. 2013). Many dust devil hot spots, including those mentioned, are located at or near coastal areas. When relatively cool water bodies exist near hot land-surface areas, onshore land-sea breezes can lead to extremely unstable atmospheric stratifications over land, thereby providing one of the key dust devil formation conditions.

Dust devils require not only vortices strong enough to raise dust but also the presence of a dust source at the surface. A known dust devil active area can therefore serve as a proxy for dust sources. As well as with local variations, dust sources can change with the seasons (Ginoux et al. 2012). Decadal changes in dust sources can be attributed to changes in climate or by anthropogenic means, such as land-use change (Ginoux et al. 2012). In that case, aridity can be affected over vast regions when sensitive to vegetation changes, such as in the Sahel (Cowie et al. 2013). Reduced vegetation can also decrease surface roughness, which in turn can lead to an increase in dust devil frequency of occurrence under certain conditions (Lyons et al. 2008). The uncertainty of dust devil occurrence on Earth caused by uncertainty of dust sources is discussed in Section 4.1.

\subsection{Dust devil occurrence frequency based on observations}

Lorenz (2014) showed with a Monte Carlo model that field meteorological observations of vortices on a terrestrial desert playa (about $300 \mathrm{~km}^{-2}$ day $^{-1}$, for a vortex detection threshold of $20 \mathrm{~Pa}$ ) are rather consistent with the number of vortices detected in LES simulations by Raasch and Franke (2011) and Ohno and Takemi (2010) when a -1 cumulative power-law (-2 differential) in core pressure drop was assumed. Specifically, those simulations indicated about 4,500 and 1,600 vortices per $\mathrm{km}^{2}$ per day, but with core pressure drops of more than 4 and $10 \mathrm{~Pa}$ and maximum vorticities of more than $1 \mathrm{~s}^{-1}$ and $0.15 \mathrm{~s}^{-1}$, respectively. The Monte Carlo results indicate that vortices are more abundant than visually-observed dust devils, which at that site (Eldorado Playa) occurred with a frequency of about $100 \mathrm{~km}^{-2} \mathrm{day}^{-1}$. This is readily under- 
stood as a dust devil intensity threshold for dust lifting to occur, expressed in pressure terms as about $0.3-0.8 \mathrm{hPa}$ (regardless of the actual dust-lifting mechanism and its dependence on shear stress, pressure drop or other factors, it is convenient to assume these factors are proportional to pressure drop), not too different from results of laboratory measurements. 


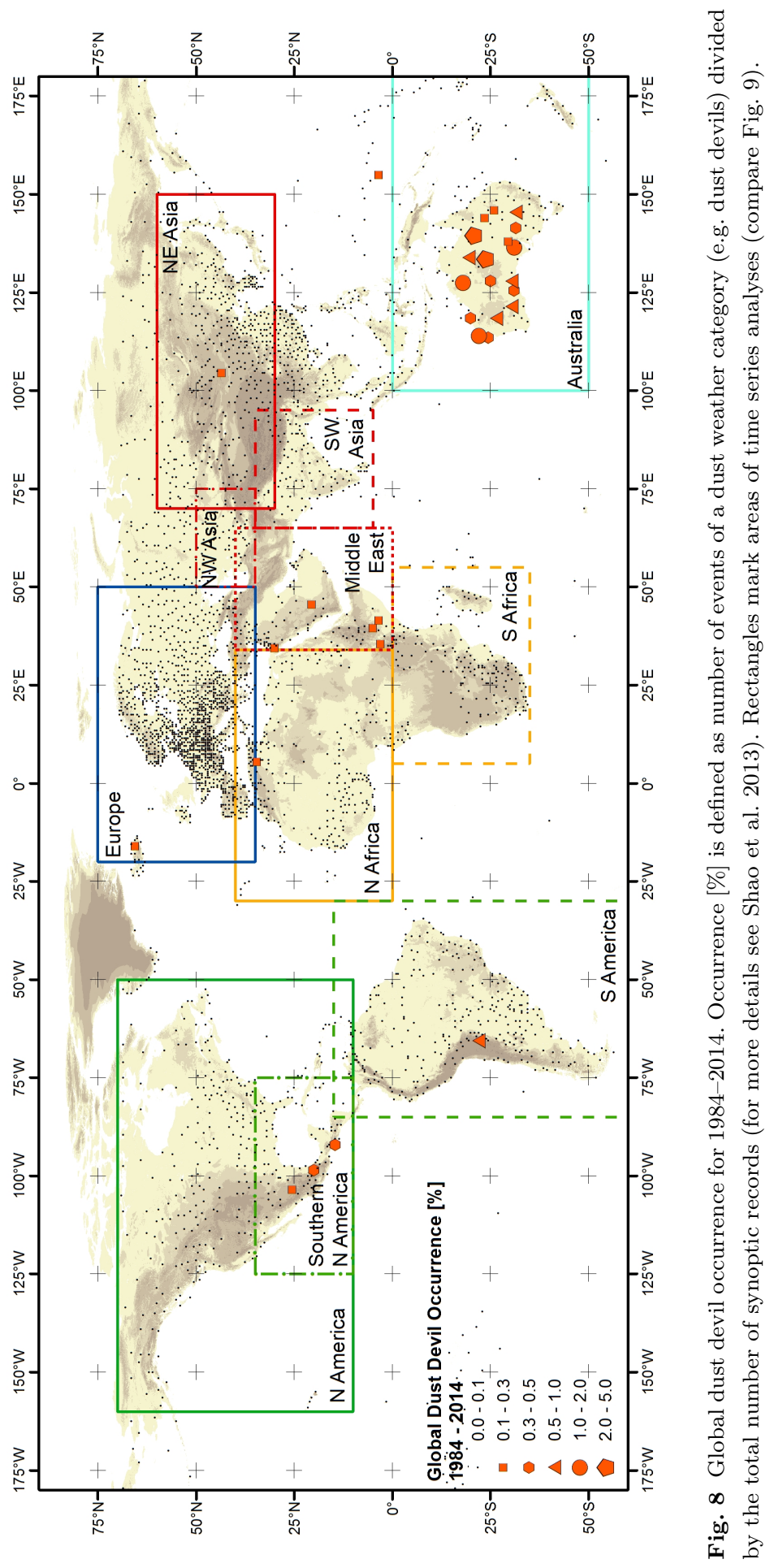


Shao et al. (2013) analyzed global synoptic weather reports and calculated time series of dust event occurrence for different regions of the world for the categories of weak dust events (present weather reported as being blowing dust or dust in suspension), strong dust events (dust storm or severe dust storm), and other dust events (dust devils, thunderstorm with dust or dust during the past 3 or 6 hours). Occurrence [\%] is defined as $N_{\mathrm{w}} / N_{\mathrm{obs}}$, where $N_{\mathrm{w}}$ is the number of events of a dust weather category (e.g. dust storm) and $N_{\text {obs }}$ is the total number of synoptic records. Only present and past weather records of manned stations are considered (for more details see Shao et al. 2013). Klose (2014) augmented this analysis by treating the category for dust devils separately. Figure 8 shows the global occurrence of dust devils for the time period of $1984-2014$. The highest frequencies of dust devil occurrence are restricted to remarkably few regions. Australia stands out with by far the highest occurrence frequencies. It cannot be guaranteed that observers report present weather consistently at stations all over the world, however, so the results should be interpreted with caution. Other regions with high reported dust devil occurrence frequencies are East Africa and the Middle East, Iceland, Southern America, and southern North America (compare Figure 7). Compared to the categories of weak, strong, and other dust events, only Australia and southern North America show significant dust devil frequencies (Figure 9). For Australia, the dust devil frequency mostly exceeds that of strong and other dust events. Only dust in suspension and blowing dust (weak dust events) are reported more often. Particular peaks in the frequency of strong dust events illustrate continent-wide dust storms, e.g. in September 2009 (De Deckker et al. 2014). In southern North America, dust devils are not generally the dominant dust event category, but can be in particular years, such as in 1999 or to a lesser extent 1994. Globally, a seasonal cycle of dust devil frequency can be recognized, which shows regular peaks in southern hemispheric spring and summer and is thus probably strongly related to the Australian records. See also Chap. 1 (Lorenz et al., 2016) for reports on dust devil activity by geographical area.

On Mars, the images taken by the MER Spirit rover are again the only statistically significant data set (Greeley et al. 2006, 2010). During the first full dust devil season (i.e., southern spring and summer) of Spirit operations, there were 51 dust devils $\mathrm{km}^{-2} \mathrm{sol}^{-1}$ in Gusev crater (Greeley et al. 2006). 

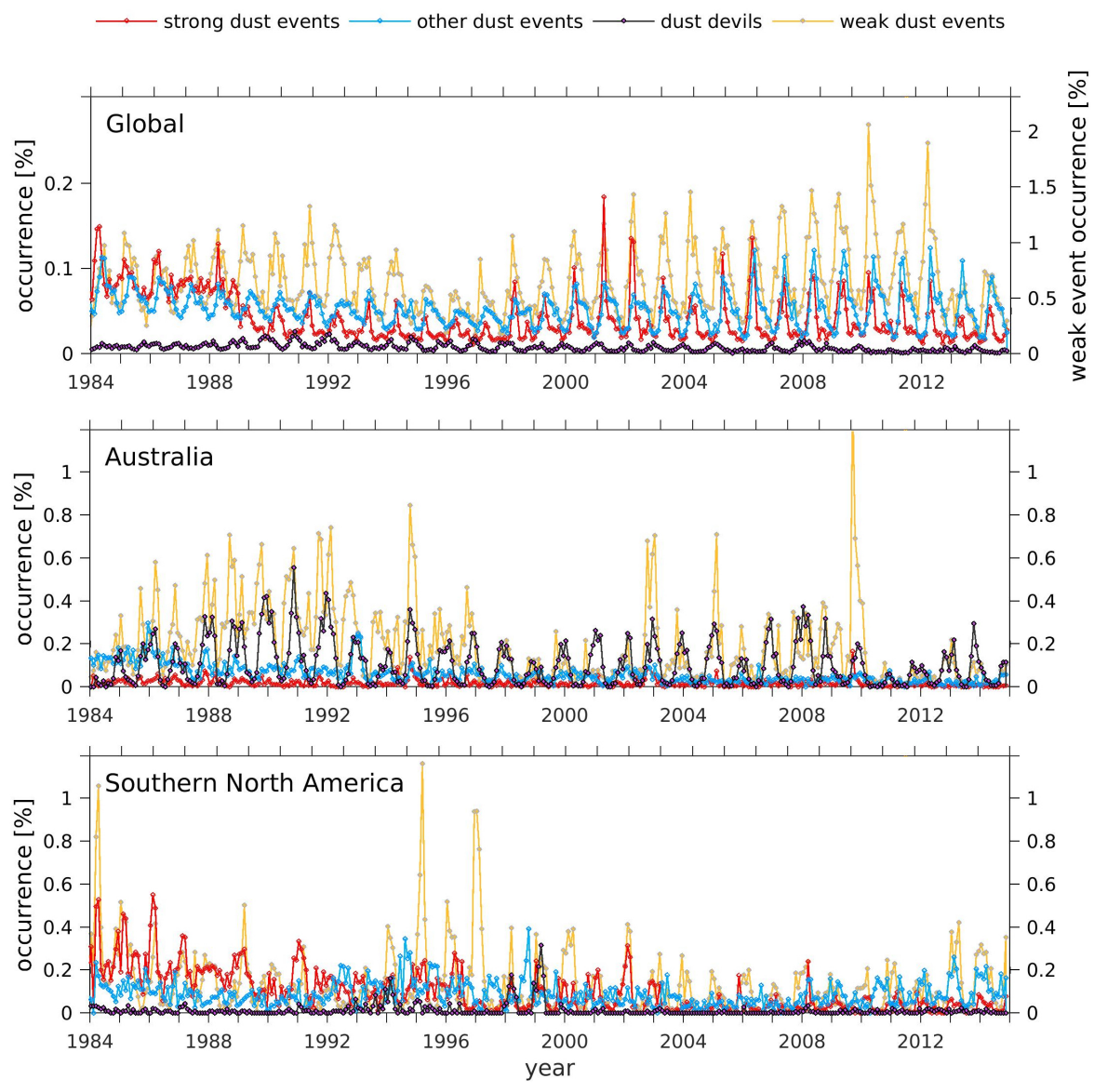

Fig. 9 Time series of dust event occurrence globally, for Australia, and southern North America (analysis domains are shown in Fig. 9). Note the different axes scales for the global time series (after Shao et al. 2013).

These numbers dropped to respectively 11 and 20, however, in the two subsequent dust devil seasons. Inter-annual differences in dust devil frequency are attributed to variations in atmospheric opacity due to regional dust storms that change the local thermodynamic conditions, with increased atmospheric dust content tending to reduce e.g. the surface sensible heat flux and thus reduce the number of dust devils present (Greeley et al. 2010; Lemmon et al. 2015).

Several studies used dust devil track density to infer global dust devil frequency (for detailed information about dust devil tracks, see Chap. 4 [Reiss et al., 2016]). Dust devil activity was found to have a latitudinal dependence, 


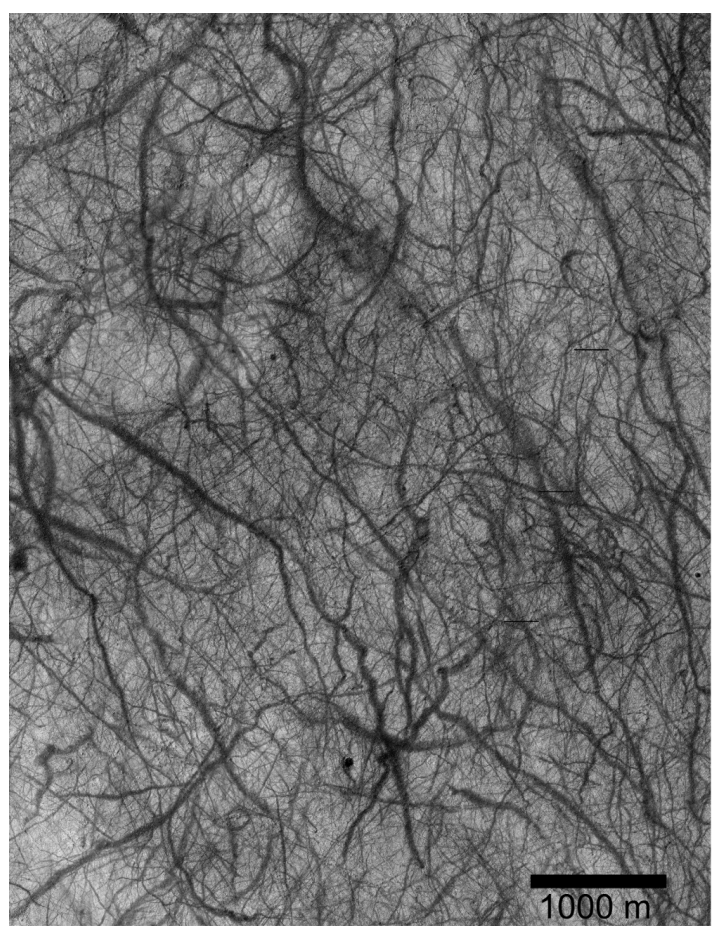

Fig. 10 Hundreds of ephemeral dust devil tracks (widths up to $100 \mathrm{~m}$ ) left by passages of dust devils, giving an impression on how numerous dust devils occur on Mars. HiRISE image ESP_013538_1230 at $56.8^{\circ} \mathrm{S}$ and $198.1^{\circ} \mathrm{E}$

with the southern hemisphere producing more dust devils than the north (Whelley and Greeley 2006). Dust devil track (ddt) densities are $9 \times 10^{-5}$ and $9 \times 10^{-4} \mathrm{ddt} \mathrm{km}^{-2} \mathrm{sol}^{-1}$ for the northern and southern hemisphere, respectively. Furthermore, $55 \%$ of activity in the northern hemisphere is limited to between 45 and $75^{\circ} \mathrm{N}$ during northern spring and summer; while $65 \%$ of the dust devil activity during southern spring and summer is limited to between 45 and $75^{\circ} \mathrm{S}$ (Whelley and Greeley 2008).

Regional studies in Argyre and Hellas Planitiae by Balme et al. (2003) show dust devil track densities of $1.2 \times 10^{-3}$ and $7 \times 10^{-4} \mathrm{ddt} \mathrm{km}^{-2} \mathrm{sol}^{-1}$, respectively. These regional dust devil track densities are in good agreement with densities of $9 \times 10^{-4} \mathrm{ddt} \mathrm{km}^{-2} \mathrm{sol}^{-1}$ for the southern hemisphere measured by Whelley and Greeley (2006). Local studies using multi-temporal high resolution remote sensing data reveal higher dust devil track densities. Verba et al. (2010) analyzed dust devil tracks in Gusev and Russell crater 


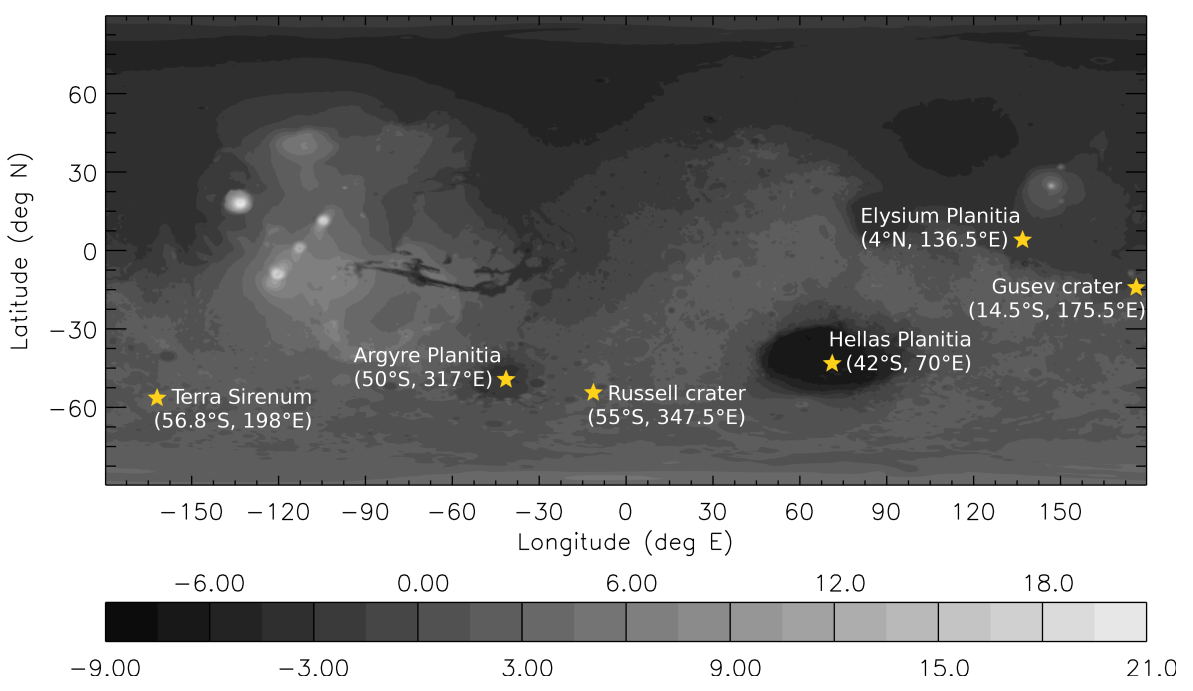

Fig. 11 Map of Mars showing topographic height above the geoid $[\mathrm{km}]$ as measured using the Mars Orbiter Laser Altimeter on board the Mars Global Surveyor (MGS) spacecraft. Locations of dust devil track studies are marked.

and found averaged dust devil track formation rates of $3.4 \times 10^{-2}$ and $2.5 \times$ $10^{-1} \mathrm{ddt} \mathrm{km}^{-2} \mathrm{sol}^{-1}$. Formation rates at the future InSight landing site region in Elysium Planitia are with $4.6 \times 10^{-2} \mathrm{ddt} \mathrm{km}^{-2} \mathrm{sol}^{-1}$ similar to those at Gusev crater (Reiss and Lorenz 2015). Figure 10 shows ephemeral dust devil tracks imaged by HiRISE in Terra Sirenum in early southern summer and gives an impression on how numerous dust devils occur on Mars. The study locations given in this paragraph are shown in Figure 11.

The dust devil frequencies inferred from dust devil tracks (Balme et al. 2003; Whelley and Greeley 2006, 2008; Verba et al. 2010; Reiss and Lorenz 2015) are much lower in comparison with the statistically significant data obtained by the MER Spirit rover in Gusev crater (Greeley et al. 2006, 2010). In general, inferring dust devil frequency from dust devil tracks is problematic. Global remote sensing observations of active dust devils leaving tracks (Cantor et al. 2006) as well as the comparison of dust devil track with active dust devil frequencies (Verba et al. 2010; Greeley et al. 2010) in Gusev crater showed that only a fraction of dust devils leave surface tracks. The formation of dust devil tracks depends on several parameters such as dust availability, dust cover thickness and substrate properties (Balme et al. 2003; Fisher et al. 
2005; Whelley and Greeley 2006, 2008). More detailed discussion is provided in Chap. 4 (Reiss et al., 2016).

The occurrence frequency of martian dust devils has also been studied by identifying dust devils in images taken from orbit (Cantor et al. 2006; Reiss et al. 2014). In contrast to the surveys based on dust devil tracks, these surveys found the dust devil activity to be stronger in the northern hemisphere. For example, $88.5 \%$ of the dust devils identified in the comprehensive survey by Cantor et al. (2006), reporting 11,456 dust devil observations, occurred in the northern hemisphere. The difference is probably explained by detection bias. Only the largest dust devils can be directly seen from orbit, but tracks left by all sizes are visible. Some areas in the northern hemisphere, especially Amazonis Planitia, are known as breeding grounds of dust devils with monumental dimensions (Fenton and Lorenz 2015). These areas probably distort the statistic of dust devils directly observed from orbit. Based on the surveys of Fisher et al. (2005) and Cantor et al. (2006) in Amazonis Planitia, dust devil frequencies are around $6.3 \times 10^{-4}$ and $5.2 \times 10^{-4} \mathrm{ddt} \mathrm{km}^{-2} \mathrm{sol}^{-1}$, respectively. However, these very low dust devil frequencies do not represent real dust devil frequencies and are orders of magnitude underestimations, because satellite imagery only provides snapshots of the daily activity and image resolutions do not resolve smaller dust devils, which occur much more frequently.

3.3 Dust devil occurrence frequency based on numerical models

\subsubsection{Large-eddy simulation}

In LES, dust devils can be identified from their characteristic properties such as pressure drop, $\Delta p$, and vorticity, $\zeta$, and are detected using threshold criteria for the latter quantities. Ohno and Takemi (2010) (OT10) and Raasch and Franke (2011) (RF11) conducted LES simulations for terrestrial conditions with a surface sensible heat flux of $H=290 \mathrm{~W} \mathrm{~m}^{-2}$ and zero mean wind using $3 \mathrm{~m}$ and $2 \mathrm{~m}$ horizontal resolution, respectively. Klose and Shao (2016) (KS16) conducted a set of terrestrial simulations with varying surface heat fluxes and wind speeds at $10 \mathrm{~m}$ horizontal resolution. During $2000 \mathrm{~s}$ of simulation time in a $1 \mathrm{~km}^{2}$ domain, OT10 identified 225 dust devils with pressure drops exceeding a threshold value of $10 \mathrm{~Pa}$ and maximum vorticities of more than $0.15 \mathrm{~s}^{-1}$ at $10 \mathrm{~m}$ height, corresponding to a dust devil number of about $400 \mathrm{~km}^{-2} \mathrm{~h}^{-1}$. 
RF11 found 25,000 dust devils during $5400 \mathrm{~s}$ in a $16.8 \mathrm{~km}^{2}$ domain, or about $1000 \mathrm{~km}^{-2} \mathrm{~h}^{-1}$, by applying a perturbation pressure threshold of $4 \mathrm{~Pa}$ and a vorticity threshold of $1 \mathrm{~s}^{-1}$ at about $1 \mathrm{~m}$ height. Varying threshold criteria have been tested by KS16. With thresholds close to those applied by OT10 (10 Pa and $0.1 \mathrm{~s}^{-1}$ at $10 \mathrm{~m}$ ), KS16 found 25 dust devils during 1 hour in a $4 \mathrm{~km}^{2}$ domain for a simulation with $H=200 \mathrm{~W} \mathrm{~m}^{-2}$ and weak mean wind $\left(u_{*}=0.15 \mathrm{~m} \mathrm{~s}^{-1}\right)$, and 100 for $H=400 \mathrm{~W} \mathrm{~m}^{-2}$, yielding 6 to $25 \mathrm{~km}^{-2} \mathrm{~h}^{-1}$. KS16 found no dust devils when using criteria of $5 \mathrm{~Pa}$ and $1 \mathrm{~s}^{-1}$ at $2 \mathrm{~m}$ height, close to those used by RF11. It is likely that the lower number of detected dust devils by KS16 is due to their coarser model resolution. Vorticities as large as $1 \mathrm{~s}^{-1}$ are thus barely reached. For example, RF11 identified twice as many dust devils in a simulation with $1 \mathrm{~m}$ horizontal resolution rather than $2 \mathrm{~m}$. Additionally, KS16 have applied a post processing on the identified dust devil tracks. In this post processing, short $(<30 \mathrm{~s})$ tracks were deleted and tracks belonging to the same vortex were connected by removing gaps arising from values of $\Delta p$ and $\zeta$ that intermittently did not satisfy the threshold criteria. This further reduced the number of dust devil counts in the study of KS16. Despite being lower than those found by OT10 and RF11, the change in the number of detections as the threshold criteria are varied is consistent in the study of KS16 with the power-law relationship between dust devil frequency and pressure drop suggested by Lorenz (2014) (compare Section 3.2).

Rafkin et al. (2001) presented the first LES results for Mars using their Mars Regional Atmospheric Modeling System (MRAMS). The model was set up with a $100 \mathrm{~m}$ horizontal resolution for a $18 \times 18 \mathrm{~km}^{2}$ domain. Rafkin et al. (2001) initialized the LES with a pre-sunrise thermodynamic sounding obtained from a $2 \mathrm{D}$ model version and vertically increasing background wind profile. By detecting local vorticity maxima and pressure minima, Rafkin et al. (2001) found a density of dust-devil like vortices of $0.03-0.1 \mathrm{~km}^{-2}$ at any given time during the simulation time of one half sol. Gheynani and Taylor (2011) conducted two-hour simulations for the Phoenix lander site using the NCAR LES model (Sullivan et al. 1994) with a horizontal resolution of $25 \mathrm{~m}$, a horizontal domain size of $5 \times 5 \mathrm{~km}^{2}$, and a vertically stretched grid. Vortices were identified in their simulations by searching for corner velocity vectors that follow a circular motion. Using a surface sensible heat flux of $24 \mathrm{~W} \mathrm{~m}^{-2}$, Gheynani and Taylor (2011) found that dust devil density decreased with increasing 
background wind speed and obtained densities of $2.32,0.88$, and $0.08 \mathrm{~km}^{-2}$ for geostrophic winds of 0,4 , and $8 \mathrm{~m} \mathrm{~s}^{-1}$ without and $1.96,0.92$, and $0.08 \mathrm{~km}^{-2}$ with radiative forcing included in their simulations.

\subsubsection{Regional and global models}

Due to the coarser spatial resolution of $10^{0}-10^{2} \mathrm{~km}$, dust devils are not resolved in regional and global atmospheric models of Earth and Mars. As the focus of terrestrial dust modeling has so far almost exclusively been on regional- and large-scale events such as dust storms, no studies exist that provide an explicit estimate of dust devil frequency using meso- and large-scale numerical models. However, it is possible to estimate dust devil frequency from regional and global model data based on LES results. Klose and Shao (2016) conducted experiments for 15 different atmospheric stability and background wind conditions and obtained a relationship between the number density of dust devils, $n=N /(A T)$, where $N$ is the number of dust devils detected in an area $A$ during a time period $T$, and Richardson number, $R i$ (calculated for $10 \mathrm{~m}$ height):

$$
n=\beta \cdot R i^{2} \quad(\text { with } R i<0)
$$

with $\beta \approx 5.8 \mathrm{~km}^{-2} \mathrm{~h}^{-1}$. The coefficient $\beta$ was found to decrease rapidly for the weakest dust devil identification criteria (i.e. small $\Delta p_{t}$ and $\zeta_{t}$ ), but to be approximately constant for a range of threshold criteria with $\Delta p_{t}>20 \mathrm{~Pa}$ or equivalently about $\zeta_{t}>0.5 \mathrm{~s}^{-1}$. Equation (5) is a robust average of the relationships obtained based on the various identification threshold and is thus more representative of the larger dust devils. With Equation (5), it is possible to estimate the number of dust devils occurring in a given area, e.g. a grid box of a regional or global model, only from modeled $R i$.

Applying this approach to results of regional or global simulations yields maps of dust devil occurrence numbers. Klose (2014) conducted simulations for Australia for July 2007 - June 2008, a time period with high dust devil and low dust storm occurrence (see Fig. 9b). Equation (5) was obtained from LES results using an idealized setup, and does not account for how the relationship might vary with changes in vegetation cover. Further LES simulations would be required to investigate this relationship. The number of dust devils is in general negatively correlated with fractional vegetation cover, $\sigma$, although small cover fractions do not preclude and might even enhance dust 


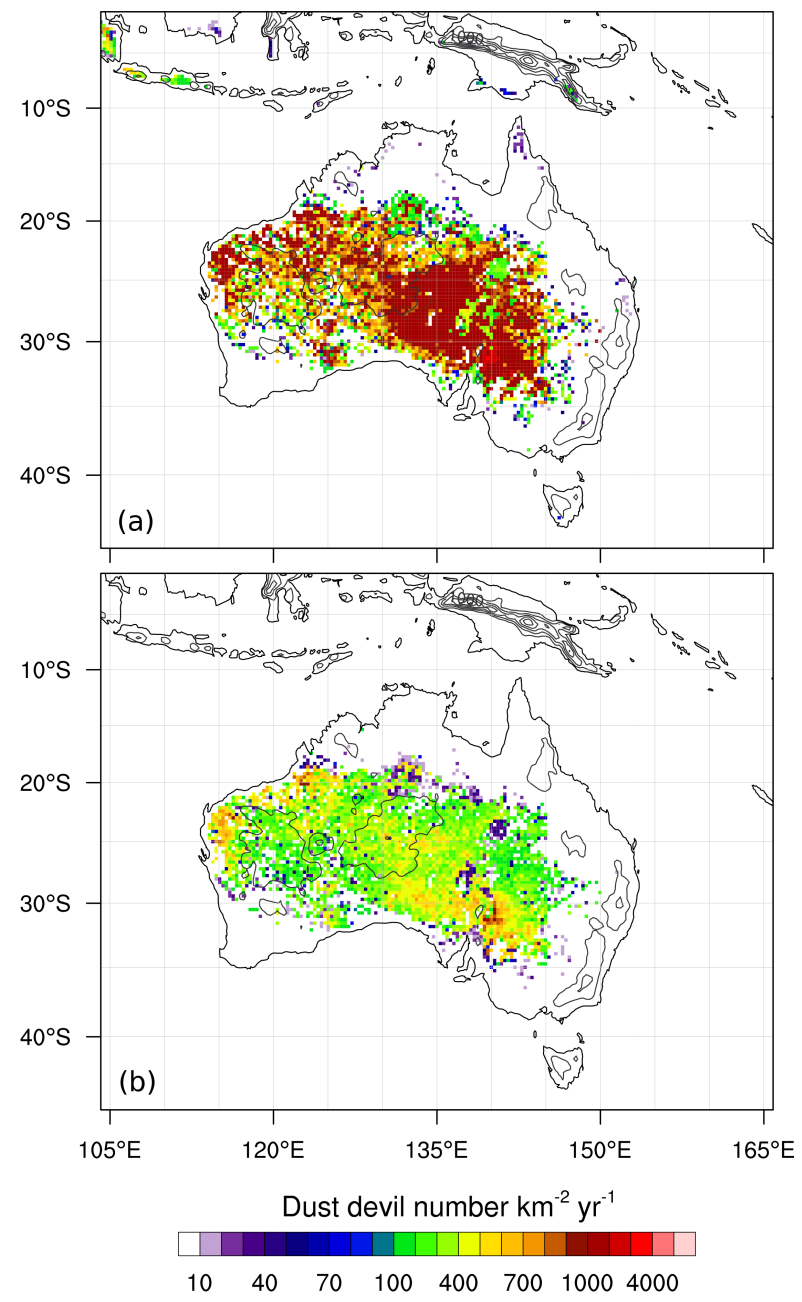

Fig. 12 (a) Estimated total number of dust devils occurring in Australia from July 2007 to July 2008 based on Equation (5) with correction for vegetation cover: $N_{\sigma}=N(1-\sigma)$; (b) as (a), but using $N_{L}$, i.e. numbers are only computed at times when the lapse rate between the surface and $2 \mathrm{~m}$ height exceeds $8.5 \mathrm{~K} \mathrm{~m}^{-1}$.

devil development (Balme and Greeley 2006; Oke et al. 2007b; Neakrase and Greeley 2010a). To account for vegetation cover, a preliminary approximation of $N_{\sigma}=N(1-\sigma)$ was made by Klose and Shao (2016). In addition, $N_{\sigma}$ was only computed for areas with less than $50 \%$ vegetation cover as it is assumed that areas with larger cover fractions are no dust sources. A clear seasonal dependence of the number of dust devils was found, with the largest numbers 
occurring in southern hemispheric spring and summer. Without the vegetation correction, dust devil numbers of about $100-200 \mathrm{~km}^{-2} \mathrm{mon}^{-1}$ were predicted over wide regions of central Australia from October to January, with maxima of up to $500 \mathrm{~km}^{-2} \mathrm{mon}^{-1}$ in the western part of Western Australia and in a region stretching from eastern South Australia (SA) to western New South Wales (NSW). After accounting for vegetation cover, the predicted dust devil numbers are generally smaller than $100 \mathrm{~km}^{-2} \mathrm{mon}^{-1}$ in central Australia, with maxima of $300 \mathrm{~km}^{-2} \mathrm{mon}^{-1}$ at few locations in the aforementioned areas. $N_{\sigma}$ for the whole year of simulation is shown in Figure 12a. Dust devil numbers in the areas of high activity integrate to about $900-1000 \mathrm{~km}^{-2} \mathrm{yr}^{-1}$. Very few grid points in eastern SA and western WA show numbers of up to $4000 \mathrm{~km}^{-2} \mathrm{yr}^{-1}$.

Oke et al. (2007b) conducted a dust devil census at Fowlers Gap research station in western NSW, close to the dust devil hot spot in eastern SA identified here. During their 20 day observation period in January 2001, Oke et al. (2007b) counted 557 dust devils in a $35 \mathrm{~km}^{2}$ area, translating to about $25 \mathrm{~km}^{-2} \mathrm{mon}^{-1}$. For January 2008, the approach described above yielded $N_{\sigma} \approx 32 \mathrm{~km}^{-2} \mathrm{mon}^{-1}$ in the corresponding model grid cell, slightly larger than the number observed by Oke et al. (2007b). More dust devils have been reported in Australia in 2008, the time of simulation shown here, compared to 2001, when the census was conducted by Oke et al. (2007b) (Fig. 9 ), so the results are plausible. However, $N_{\sigma}$ is larger in the surrounding grid cells. Oke et al. (2007b) found that no dust devils occurred for near surface temperature lapse rates lower than $\sim 1 \mathrm{~K} \mathrm{~m}^{-1}$. If we apply a minimum lapse rate of $1 \mathrm{~K} \mathrm{~m}^{-1}$ as an additional criterion to our estimates, we find negligible difference in our results, thus showing the applicability of $R i$ as sole indicator for dust devil occurrence. However, if a value of $8.5 \mathrm{~K} \mathrm{~m}^{-1}$ is applied as suggested by Ansmann et al. (2009), then the dust devil number with lapse rate criterion, $N_{L}$, is smaller with $5 \mathrm{~km}^{-2} \mathrm{mon}^{-1}$ in the model grid cell corresponding to Fowlers Gap, again with larger values in the surrounding. A case-based comparison would be necessary to further investigate the criteria and validate the approach. On an annual basis, $N_{L}$ yields values of mostly below $400 \mathrm{~km}^{-2} \mathrm{yr}^{-1}$ and maxima of about $2000 \mathrm{~km}^{-2} \mathrm{yr}^{-1}$ in the westernmost part of WA and in the area surrounding Fowlers Gap (Figure 12b).

Global models for Mars have a much stronger focus on dust devils than is the case for terrestrial models. Martian global climate models (GCMs) con- 


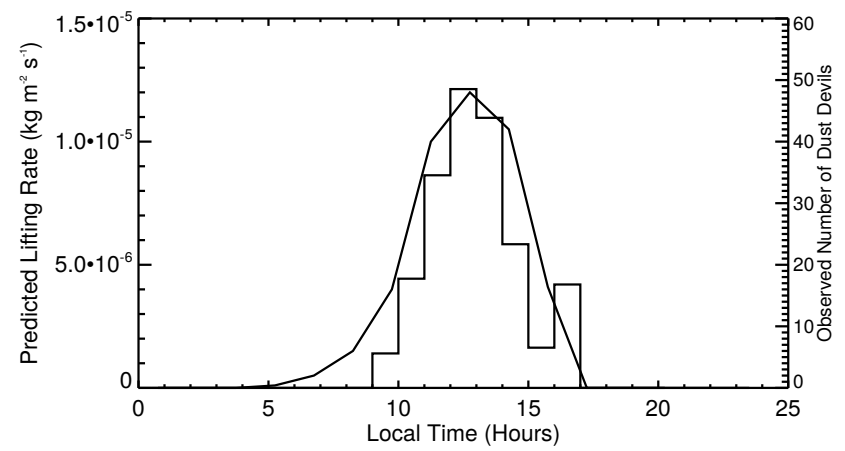

Fig. 13 Modeled diurnal dust devil lifting rate shown for the model grid point nearest the Mars Pathfinder landing site (solid curve) and histogram of observed diurnal occurrence of dust devils during the Pathfinder mission (Murphy and Nelli 2002). (Reprinted from Kahre et al. 2006, with permission from John Wiley and Sons)

tain separate dust devil parameterizations (Newman et al. 2002a). In these parameterizations, a positive surface heat flux and a nonzero PBL thickness is required for dust devils to occur (see Section 4.2.2). Models therefore predict that dust devils occur only during the day, with a peak activity during the early afternoon (Newman et al. 2002a; Basu et al. 2004; Kahre et al. 2006). In particular, Kahre et al. (2006) demonstrated that the predicted diurnal peak in dust devil emission rates at the Mars Pathfinder site during the season of the mission should have occurred between 12:00 and 13:00 LTST (Figure 13), which is consistent with the observed peak in pressure dips from the pressure sensor on Mars Pathfinder (Murphy and Nelli 2002). Greeley et al. (2010) analyzed dust devils over three consecutive years as observed by the Spirit lander at Gusev Crater. They found that dust devils peaked over the entire period 12:00 - 15:00LTST, in the second year featuring a later peak from 14:00 15:00 LTST, in the presence of higher dust loading, and in the third year there were significant numbers as late as 16:00 - 17:00 LTST. These were consistent with the diurnal surface heating cycle, although selection issues should also be noted in sampling.

A seasonal trend in dust devil activity for Mars is also predicted in global models because both the surface-to-atmosphere temperature difference (and thus the heat flux) and the depth of the PBL are maximized near the subsolar point. Predicted peak dust emission rates occur during local spring and summer, which is consistent with surveys of observed dust devils and dust 

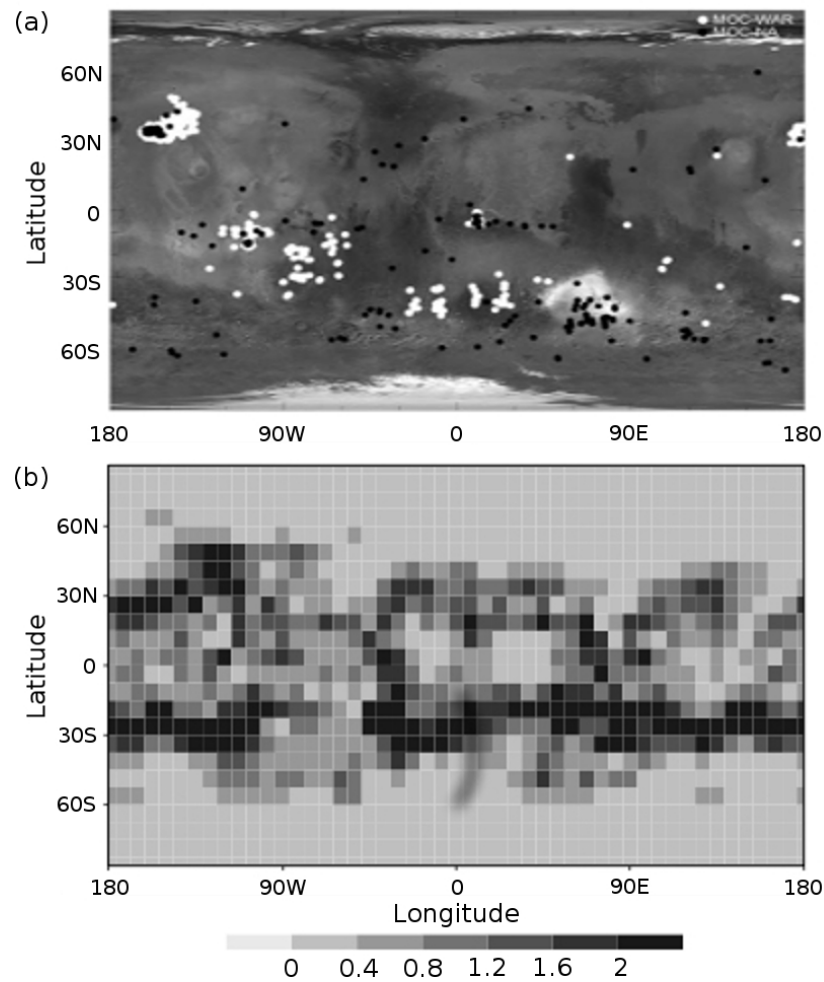

Fig. 14 (a) Afternoon spatial distribution of dust devils as observed by Mars Orbiter Camera plotted on a cylindrical map (Reprinted from Cantor et al. 2006, with permission from John Wiley and Sons). White and black dots indicate dust devils detected with two different cameras. (b) Annual dust devil dust lifting (arbitrary units) obtained from a Mars global model with only parameterized dust devil lifting (set proportional to the dust devil activity defined in Equation (6); see Section 4.2.2 and radiatively active dust (Reprinted from Newman et al. 2002a, with permission from John Wiley and Sons).

devil tracks (Newman et al. 2002a; Basu et al. 2004; Fisher et al. 2005; Kahre et al. 2006; Cantor et al. 2006; Greeley et al. 2010). Both lander-based (Greeley et al. 2010) and orbital (e.g. Cantor et al. 2006) surveys have shown that the dust devil activity ceases almost completely during local fall and winter. By contrast, most dust devil parameterizations used in Mars climate models predict some lifting whenever thermal conditions are favorable for dust devils to occur, hence lifting occurs year-round. This suggests such parameterizations do not include all the physics required to predict dust devil occurrence and/or their ability to lift dust, such as the correct formulation of a threshold intensity that must be achieved before lifting can occur. However, the total 
Table 3 Total dust transport by dust devils.

\begin{tabular}{ccll}
\hline Dust transport $\left[\mathrm{kg} \mathrm{yr}^{-1}\right]^{1}$ & Method & Note & Reference \\
\hline Earth & & & \\
$566 \times 10^{9}$ & field/theory & global & Koch and Renno (2005) \\
$59 \times 10^{9}$ & field/theory & global & Jemmett-Smith et al. (2015) \\
$7 \times 10^{9}$ & field & USA & Gillette and Sinclair (1990) \\
$0.2-1.1 \times 10^{8}$ & modeling & AUS & Klose and Shao (2016) \\
Mars & & & \\
$5.8 \times 10^{11}$ & orbiter & global & Cantor et al. (2006) \\
$2.3 \times 10^{11}$ & orbiter/lander & global & Whelley and Greeley (2008) \\
\hline
\end{tabular}

dust lifting produced by such parameterizations, which is typically tuned to match the observed background dust loading, may be accounting for other small scale lifting phenomena not represented in the model, as discussed further in Sections 4.2.2 and 5.3.2. The spatial distribution of dust devil activity predicted by the Mars climate models over a full Mars year is rather similar to orbital observations (Figure 14), particularly in terms of the overall hemispheric asymmetry (more in the southern hemisphere, which has the more intense summer) and the basic latitudinal distribution (though the peak activity is observed at slightly higher latitudes than predicted). The model also captures some of the regional trends, e.g. the greater dust devil intensity in Amazonis Planitia, although the agreement is not as good in some regions, for example the model does not capture the peak between Solis and the Tharsis Montes.

\section{Estimates of total dust transport by dust devils on Earth and Mars}

The previous sections provided insights into the dust transport associated with individual dust devils and the statistics of dust devil occurrence. In this section, these insights are combined to obtain estimates of the contributions of dust devils to regional and global dust budgets, as listed in Table 3. These estimates are either based purely on observations of dust fluxes (Section 4.1) or are based on (to a varying extent) some consideration of the physical mechanisms behind dust devil occurrence and consequently their dependence on the spatiotemporally varying state of the atmosphere in which they form (Section 4.2). 


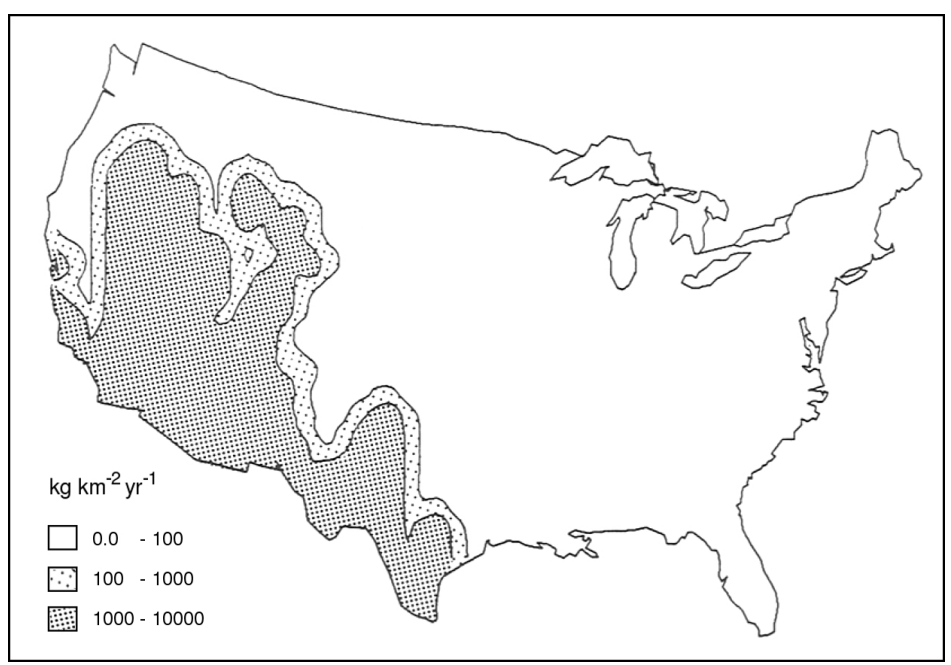

Fig. 15 Annual dust devil dust fluxes $\left(D_{p}<25 \mu \mathrm{m}\right)$ estimated by Gillette and Sinclair (1990) for the USA. Reprinted from Gillette and Sinclair (1990), with permission from Elsevier.

4.1 Total dust transport estimated from in situ and remote sensing observations

No global estimate of dust devil sediment transport on Earth is available that is based on observations only. Although regional dust devil censuses have been conducted, e.g. in Australia by Hess and Spillane (1990) and Oke et al. (2007b), in the USA by Sinclair (1969), Carroll and Ryan (1970), and Snow and McClelland (1990), or in South America by Kurgansky et al. (2011), no information on dust flux in the counted dust devils was available and thus no area estimate on transport could be obtained. The only estimate on regional dust devil transport is that of Gillette and Sinclair (1990). The authors conducted aircraft measurements in a test region near Tucson, Arizona, USA. Using these fluxes as a reference, Gillette and Sinclair (1990) assigned annual dust flux values to areas based on their climatic and vegetative conditions (Figure 15). To account for non-erodible elements on the soil surface, they further assumed a reduction of the dust flux by $50 \%$. On this basis, Gillette and Sinclair (1990) estimated that dust devils contribute $\sim 7 \times 10^{9} \mathrm{~kg}$ to the total mass of mineral dust aerosol in the contiguous USA.

1 Note that a martian year is approximately twice as long as a terrestrial year. 
On Mars, the most reliable estimates of the total amount of dust lifted on a regional scale are those based on the observations of the MER Spirit rover in Gusev grater (Sections 2.2 and 3.2). Greeley et al. (2006, 2010) combined the values of dust fluxes (Section 2.2), diameters and lifetimes of the detected dust devils to calculate total amounts of dust injected by single dust devils into the atmosphere. The results varied from $1.9 \times 10^{-4}$ to $29 \mathrm{~kg}$ per dust devil. Amounts of dust lifted per unit area were then estimated by using the average of the masses lifted by individual dust devils and the number of dust devil observations extrapolated both temporally and spatially. The value for Spirit's first dust devil season, given in Greeley et al. (2006), was $\sim 19 \mathrm{~kg} \mathrm{~km}^{-2} \mathrm{sol}^{-1}$. Instead of exact values, wide error ranges for the amount of dust lifted per unit area during the whole dust devil season were given in Greeley et al. (2010): 2.6 to $3.0 \times 10^{5} \mathrm{~kg} \mathrm{~km}^{-2}$ for season one, 4.4 to $5.3 \times 10^{3} \mathrm{~kg} \mathrm{~km}^{-2}$ for season two, and $1.5 \times 10^{2}$ to $1.6 \times 10^{5} \mathrm{~kg} \mathrm{~km}^{-2}$ for season three.

Whelley and Greeley (2008) estimated the total amount of dust lifted by dust devils on Mars using the value given by Greeley et al. (2006) for Gusev crater as ground truth and extrapolating it globally using dust devil track density observations (Section 3.2). The result was $2.3 \pm 1.0 \times 10^{11} \mathrm{~kg}$ per martian year, approximately half as much as local and regional dust storms.

Another estimate of the annual dust devil flux was calculated by Cantor et al. (2006), who estimated the occurrence frequency of dust devils using orbital observations of active vortices (Section 3.2). The dust lifting rate was calculated by determining dust devil optical depths from orbital images and assuming a $2 \mathrm{~m} \mathrm{~s}^{-1}$ vertical wind speed, an early result of Greeley et al. (2006). The estimated global mean dust flux was $4 \times 10^{3} \mathrm{~kg} \mathrm{~km}^{-2}$ per martian year. Multiplying this with the surface area of Mars yields $5.8 \times 10^{11} \mathrm{~kg}$ per martian year. This is surprisingly close to the above estimate by Whelley and Greeley (2008), considering the difference in methodology, but this may of course be coincidental.

4.2 Total dust transport estimated based on the atmospheric state

The studies presented in this section vary greatly, but all include at least some dependence on the atmospheric (and surface) state - and its control on dust devil occurrence and dust flux - in making their estimates of regional or global 
dust transport by dust devils. Conceptually, they all follow the same formula: total dust devil lifting $=$ a scaling factor $\times$ a function of the atmospheric (and surface) state. In some studies, the scaling factor is based on upscaling field measurements of individual dust devil fluxes (e.g. Koch and Renno 2005; Jemmett-Smith et al. 2015), while in others it is based on requiring that the total global dust loading due to dust devils matches the observed background dust load (e.g. for Mars Basu et al. 2004; Kahre et al. 2005), or based on explicit modeling of dust lifting by individual vortices (Klose and Shao 2016). In some studies, the function is continuous, implying a dust devil strength or intensity (e.g. Koch and Renno 2005; Newman et al. 2002a), while in others it is discrete, predicting only that dust devils would or would not occur (e.g. Jemmett-Smith et al. 2015). Finally, in some studies, the atmospheric state is obtained from observations (e.g. Koch and Renno 2005), while in others it is taken from a model (e.g. Jemmett-Smith et al. 2015; Klose and Shao 2016). While the studies overlap considerably in terms of their various inherent assumptions and simplifications, this section is divided up according to the scaling factor used.

\subsubsection{Scaling dust fluxes based on field measurements of individual vortices} (Earth)

The first global estimate for dust devil dust transport on Earth was achieved by Koch and Renno (2005). They assumed that individual dust devils lift dust at a rate of $0.7 \times 10^{-3} \mathrm{~kg} \mathrm{~m}^{-2} \mathrm{~s}^{-1}$, based on field measurements of dust concentration and vertical wind speed in dust devils (Kaimal and Businger 1970; Renno et al. 2004). They then used the thermodynamic theory for dust devils of Renno and Ingersoll (1996) and Renno et al. (1998) to estimate the fractional area over which dust devils should be active. The theory developed by Renno et al. (1998) describes a convective vortex (dust devil) as a heat engine that performs mechanical work against frictional dissipation. Driven by this convective heat engine, the dust devil activity, $F_{a v}$, is approximated by

$$
F_{a v} \approx \eta H
$$

where $\eta$ is the thermodynamic efficiency of the heat engine and $H$ is the surface sensible heat flux. $H$ is approximately proportional to the temperature 
difference between the surface and the near-surface air. The thermodynamic efficiency, $\eta$, is approximately $\eta=1-b$, with

$$
b=\frac{p_{s}^{\chi+1}-p_{\text {top }}^{\chi+1}}{\left(p_{s}-p_{\text {top }}\right)(\chi+1) p_{s}^{\chi}}
$$

where $p_{\text {top }}$ is the pressure at the top of the PBL, $p_{s}$ is the surface pressure, and $\chi$ is the specific gas constant divided by the specific heat capacity at constant pressure. Based on these expressions, the dust devil activity (and thus the amount of dust transported by dust devils) increases with both increasing boundary layer thickness and increasing sensible heat flux.

Koch and Renno (2005) used atmospheric sounding data from 9 locations in Algeria, China, Iran, Saudi Arabia, and the USA to calculate the fractional area, $\sigma$, covered by dust devils (Renno and Ingersoll 1996):

$$
\sigma \approx\left(\frac{\mu}{\eta}\right)^{1 / 2}\left(\frac{\Delta p_{a}}{\rho g \tau_{R}}\right)^{3 / 2}\left(\frac{F_{\mathrm{in}}}{\rho}\right)^{-1 / 2}
$$

where $\mu$ is a dimensionless coefficient, $\Delta p_{a}$ is the pressure decrease from the surface to the top of the convective PBL, $\rho$ is air density, $g$ is gravitational acceleration, $\tau_{R}$ is the radiative time scale, and $F_{\text {in }}$ is the heat input into the vortex. $\tau_{R}$ was estimated using a theoretical approximation and $F_{\text {in }}$ was obtained from measurements. Koch and Renno (2005) found that $\sigma$ varied only slightly for the locations and time periods considered $\left(\sigma \approx 3 \times 10^{-5}\right)$. Based on observations, Koch and Renno (2005) further assumed that dust devils likely occur $8 \mathrm{~h}$ per day at 72 days per year ( $80 \%$ of a three-month period) and that $40 \%$ of global arid and semi-arid areas are dust sources, leading to a global dust source area of $(1.3 \pm 0.2) \times 10^{7} \mathrm{~km}^{2}$. Using these area and time fractions of dust devil occurrence, Koch and Renno (2005) estimated that dust devils annually transport $\sim 566 \times 10^{9} \mathrm{~kg}$ of dust with an uncertainty of $18 \%$.

Jemmett-Smith et al. (2015) used the same individual dust devil fluxes $\left(0.7 \mathrm{~g} \mathrm{~m}^{-2} \mathrm{~s}^{-1}\right)$ derived from field measurements as Koch and Renno (2005). Rather than using a fixed annual occurrence time, they instead determined when and where dust devils should (or should not) occur based on the following constraints: locations with high convective buoyancy and low frictional dissipation, using a criterion suggested by Lyons et al. (2008) and a high lapse rate criterion as suggested by Ryan (1972), Oke et al. (2007b), and Ansmann et al. (2009) (see Section 3.1 for more details). Jemmett-Smith et al. (2015) applied their criteria truly globally using high-resolution ECMWF operational 
analyses (see Section 3.1). They also used a global dust source region mask as opposed to a global dust source fraction as used by Koch and Renno (2005). Jemmett-Smith et al. (2015) assumed the same constant fractional area to be covered by dust devils within each active region $\left(3 \times 10^{-5}\right)$ as calculated by Koch and Renno (2005). Overall, Jemmett-Smith et al. (2015) proposed that only $59 \times 10^{9} \mathrm{~kg}$ of dust is transported by dust devils globally per year, with an uncertainty range for their estimates of $\sim 2$ to $66 \times 10^{9} \mathrm{~kg}$, depending on which dust source mask was applied.

In addition to their differences in approach, the studies described above are both highly sensitive to uncertainties in the estimated individual dust devil dust fluxes, which are key to upscaling their results to produce regional and global estimates. Typical dust devil flux values given by Metzger et al. (2011) are about 2 orders of magnitude less than the values proposed by Renno et al. (2004), used by Koch and Renno (2005) and Jemmett-Smith et al. (2015) to gain their global estimates. Additionally, the fluxes calculated from measured dust concentrations and vertical wind speeds at a given height (as made in field observations of dust devils, see Section 2.2) are likely different to surface dust emission fluxes. Convergence in the dust devil vortex may lead to an overestimation of the dust flux at small heights $(\sim 2 \mathrm{~m})$, whereas turbulent diffusion may lead to an underestimation if measurements are taken at larger heights $(\sim 100 \mathrm{~m})$. If sediment coarser than dust is considered, then gravitational settling may lead to an underestimation of the coarse size-fraction even at small heights. The dust fluxes at altitude may thus not be directly transferable to surface sediment loss at a given time and location.

The explicit assumption that the characteristics of individual ('typical' or 'average') dust devils, such as the dust flux, may be 'scaled up' to provide regional and global estimates is problematic. For example, taking one field measurement of a 'typical' diameter and multiplying by some observed number of dust devils ignores the dependence of a number of parameters that scale with diameter. As noted by Lorenz (2011), for a fixed dust-lifting rate per unit area, the area occupied by a single devil varies as the square of diameter, or if advected at a constant speed, the area swept will vary with diameter raised to the $n$th power (with $n$ between 1.5 and 1.75). This exponent arises because empirically dust devil longevity varies with diameter. For example, Lorenz (2014) suggested that duration varies as roughly $40 d^{0.66}$, where $d$ is the 
diameter in meters and duration is measured in seconds, yielding an exponent of $n \approx 1.66$ for a fixed wind speed. Additional scaling factors may further increase this exponent, however; for example, if larger dust devils are also more intense, then their dust-lifting rate is also expected to be larger as laboratory experiments have shown (Section 2.1). Thus despite the strongly skewed size distribution of dust devils (lots of small ones, few large ones) (Kurgansky 2006; Lorenz 2011), the dust lifting may in fact be dominated by the rarest, largest dust devils. Hence any approach that relies on multiplying up the dust lifting by a single 'typical' (i.e. rather smaller) dust devil is unlikely to accurately represent the overall lifting by the full population (see Chap. 8 [Lorenz and Jackson, 2016] for a more detailed discussion).

Using different dust source maps, Jemmett-Smith et al. (2015) also showed that uncertainties in global dust sources affect global estimates by 1 order of magnitude. This combines with the roughly 2 orders of magnitude maximum uncertainty in the dust flux, giving up to 3 orders of magnitude uncertainty in the total estimate of global dust emission. Continued advances in satellite observations will reduce uncertainty in dust sources (Ginoux et al. 2012; Schepanski et al. 2012), which will subsequently reduce uncertainty in dust devil contributions. However, the inclusion of improved estimates of dust devil dust fluxes will lead to more substantial advances in total transport estimates given the larger uncertainties involved.

The large difference in the global estimates obtained by Koch and Renno (2005) and Jemmett-Smith et al. $(2015)\left(\sim 600 \times 10^{9}\right.$ versus $\left.60 \times 10^{9} \mathrm{~kg} \mathrm{yr}^{-1}\right)$ demonstrate the effect of the variability in surface and atmospheric conditions on dust devils and dust lifting. The major reason for the difference is likely the better spatial and/or temporal representation of both dust source areas and meteorological conditions by Jemmett-Smith et al. (2015) compared to Koch and Renno (2005). While the latter used a specified proportion of the global area to scale dust devil lifting $\left(1.3 \times 10^{7} \mathrm{~km}^{2}\right)$, Jemmett-Smith et al. (2015) used a spatially resolved dust source description. Both, Koch and Renno (2005) and Jemmett-Smith et al. (2015) used a fixed thermodynamic efficiency, obtained by Koch and Renno (2005) using atmospheric soundings for convectively active days from key dust regions, to deduce a (constant) fractional dust devil updraft area. However, while Koch and Renno (2005) used a fixed temporal fraction to correct their result for days during the active dust season which were without 
dust devils, Jemmett-Smith et al. (2015) used spatially and temporally varying critera to constrain their annual estimate. Consequently, the dust devil dust fluxes resulting from both studies differ by one order of magnitude although both employ the same dust flux value for individual dust devils.

4.2.2 Scaling dust fluxes based on global observational constraints on dust loading (Mars)

Due to the greater radiative impact of atmospheric dust in the thin martian atmosphere and the lack of oceans or large amounts of water to collect dust rapidly once lifted, dust raised into the atmosphere on Mars has a far more direct and larger scale impact on the circulation than on Earth. This likely explains why studies of dust lifting on Mars have tended to focus on representing large-scale seasonal and spatial variations in dust load and their impact on the circulation, rather than on individual dust devils or small dust lifting events. For this reason, all current estimates of transport due to dust devils on Mars - other than the purely observational estimates given in Section 4.1 - are derived from global atmospheric models, in which dust lifting parameterizations are 'tuned' such that the spatial and seasonal variation of dust loading (and/or the resultant mid-level temperatures) in the model matches that observed.

Dust devils are a larger component of the dust cycle on Mars than on Earth, because almost the entire martian planet is desert-like and typically has large near-surface lapse rates and PBL depths. As a result, lifting by dust devils has long been treated separately to lifting via near-surface wind stress in atmospheric models (e.g. Newman et al. 2002a,b). This is in contrast to the longstanding situation for Earth, as discussed in Section 4.2.3. The net result is that all estimates for dust devil transport on Mars given below have a scaling factor derived from global observational constraints on the total dust load (or its climatological effect), and depend on some function of the atmospheric and surface state as simulated by a Mars global circulation model.

To date, dust devil lifting in Mars climate models has been parameterized using functions ranging from a simple dependence on the near-surface lapse rate (e.g. Basu et al. 2004), to the 'dust devil activity' metric defined by Renno et al. (1998) or a combination of the Renno et al. (1998) thermodynamic theory with measurements of dust lifting by a laboratory-produced vortex (e.g. 
Newman et al. 2002a; Kahre et al. 2006). This first type of parameterization schemes assumes that the dust emission flux is proportional to respectively the ground-to-air temperature difference or the dust devil activity (given in Section 4.2.1). An implicit 'threshold' is applied, in that dust devil lifting shuts off when the surface is cooler than the atmosphere above it (switching off the sensible heat flux driving convection). Figure 16 shows an example of model predicted surface sensible heat flux, thermodynamic efficiency, and dust devil activity, which is proportional to dust lifting in the parameterization scheme. 


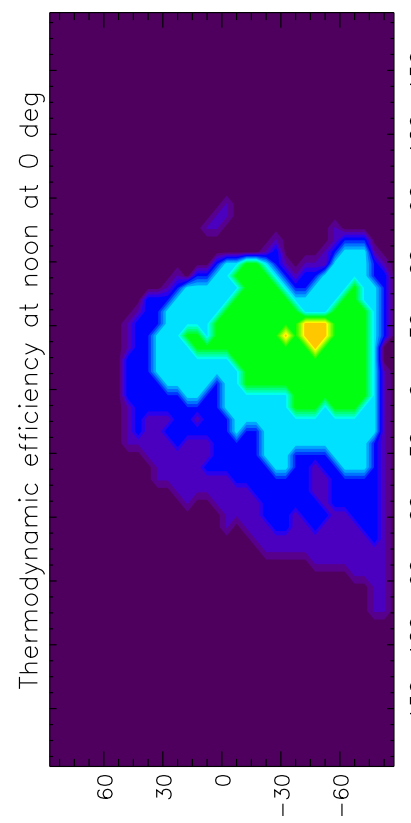

(N 6әр) әрпұ!ฺD7

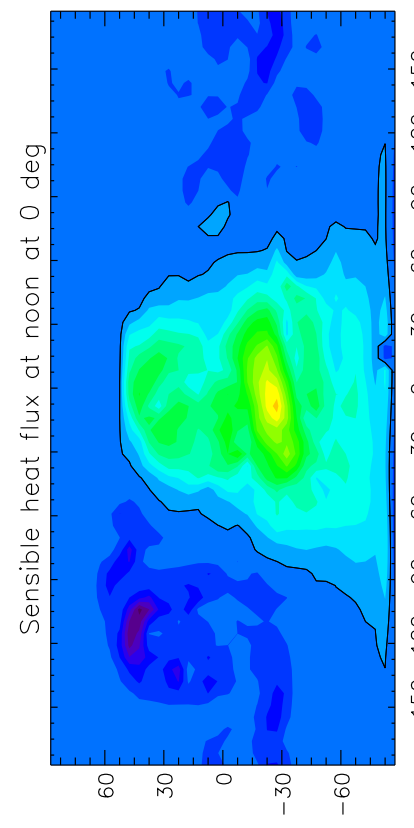

(N Бәр) әрпч!ฺ०ך

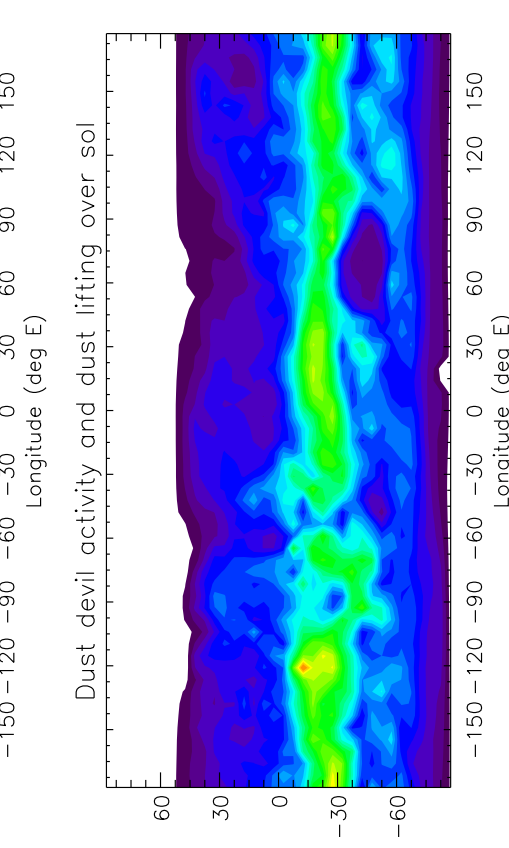

(N 6әр) әрп¥!?р7

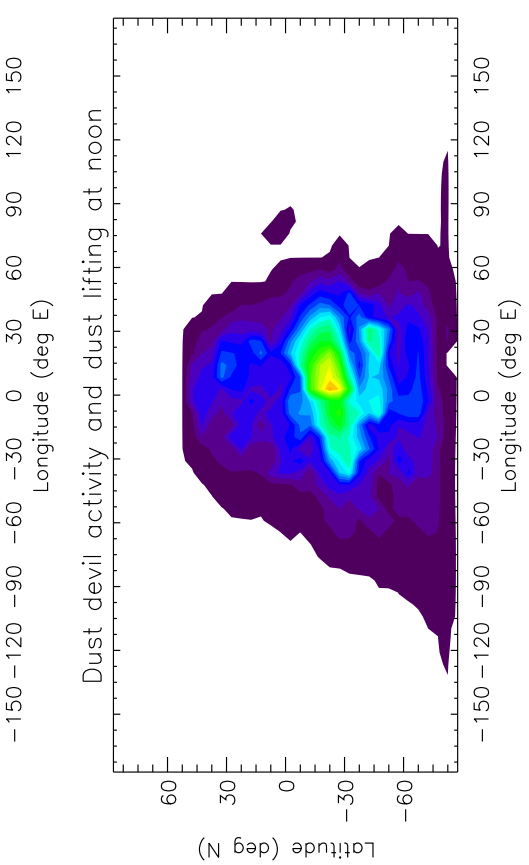

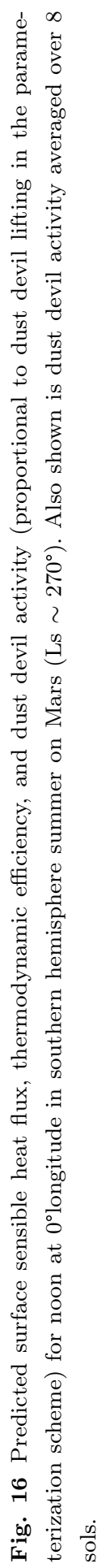


The second parameterization scheme involves a threshold based on a semiempirical formula for the tangential wind speed around the convective core that is needed for dust lifting to occur, derived from laboratory measurements of dust raising by a vortex (Greeley and Iversen 1985). The tangential wind speed around the vortex core is calculated from the model's atmospheric state via the convective heat engine model, in which the pressure drop to the vortex core, $\Delta p$, is given by

$$
\Delta p=p_{s}\left\{1-\exp \left[\left(\frac{\gamma \eta}{\gamma \eta-1}\right)\left(\frac{\eta_{H}}{\chi}\right)\right]\right\}
$$

where $\gamma$ is the fraction of the total dissipation of mechanical energy that is consumed by friction at the surface (a free parameter often set to 0.5 ), and $\eta_{H}$ is the horizontal thermodynamic efficiency of the dust devil, given by $\eta_{H}=$ $\left(T_{0}-\bar{T}_{s}\right) / \bar{T}_{s}$. In this expression, $T_{0}$ is the temperature at the center of the vortex at the surface (typically set to the ambient surface temperature) and $\bar{T}_{s}$ the temperature of the near-surface air outside the vortex.

By substituting Eq. (9) into Eq. (4), an expression for the tangential wind speed around a vortex is obtained:

$$
u_{\theta}=\sqrt{R T\left\{1-\exp \left[\left(\frac{\gamma \eta}{\gamma \eta-1}\right)\left(\frac{\eta_{H}}{\chi}\right)\right]\right\}} .
$$

Newman et al. (2002b) chose a threshold based on a semi-empirical expression for the tangential wind speed required to lift a single layer of dust in laboratory experiments by Greeley and Iversen (1985). This threshold could be chosen using another method, based on the same principles as those used for general saltation processes. However, although it is expressed as a threshold wind speed, this laboratory-based threshold also accounts for other lifting effects associated with dust devils, such as the $\Delta p$-effect and influence of electric fields (Section 2), in addition to the saltation threshold for particle movement (i.e. it is lower than the value of the saltation threshold alone). Balme and Hagermann (2006) investigated the relative importance of the $\Delta p$-effect, e.g. lifting from the reduced pressure at the core of dust devils, compared to the winds. They found that the $\Delta p$-effect was most significant when the pressure change occurred rapidly, i.e. for quickly-moving dust devils and for the most intense vortices. In general, lifting based solely on wind shear might therefore be an under-prediction, although the significance of the $\Delta p$-effect has not been 
quantified yet. The relatively higher importance of additional lifting mechanisms specific to dust devils, such as the $\Delta p$-effect, is another motivation for parameterizing dust devils separately from other sub-grid or grid-scale winds for Mars (see Chap. 10 [Neakrase et al., 2016] for more details).

Figure 17 shows the depth of dust removed by dust devils according to the first ("dust devil activity"-based) parameterization and its seasonal evolution for regions included in the dust devil survey by Fisher et al. (2005). While both of the dust devil parameterizations described above have been implemented into and used in global dust cycle studies for Mars, the first scheme has been used more consistently because it is simpler and it provides a smoothly varying background dust loading during northern hemisphere spring and summer (Newman et al. 2002b; Basu et al. 2004; Kahre et al. 2006; Mulholland et al. 2013). Additionally, it has been shown to reproduce the observed seasonal behavior of dust devil activity in both the northern and southern hemispheres (Fisher et al. 2005; Kahre et al. 2006). However, we note that both parameterizations are simplifications of the actual situation. The dust devil activity, $F_{a v}$, is a measure of the energy available for dust devils, but in itself it tells nothing about the intensity distribution of the vortex population. As weak vortices lift no dust and the amount of dust that can be lifted depends strongly on the central pressure drop (Section 2), two dust devil populations with the same $F_{a v}$, but different pressure drop distributions, would lift different amounts of dust. The second method, involving a semi-empirical formula derived from lab vortex experiments, attempts to take into account the intensity of the vortices, but it assumes that all vortices within a region have equal central pressure drops, which is unrealistic.

On Mars, dust devils are believed to be critical for maintaining the background dust loading (and thus the correct atmospheric temperatures) throughout the year, but particularly during northern hemisphere spring and summer, when very few regional and no global dust storms occur. Most models that employ both a dust devil parameterization and a saltation bombardment scheme for larger-scale dust events first tune the dust devil scheme to roughly match the northern hemisphere summer global dust loading, before tuning the saltation scheme to achieve reasonable dust storm behavior in the northern hemisphere winter (e.g. Basu et al. 2004; Kahre et al. 2006; Newman and Richardson 2015). While saltation typically contributes slightly to the back- 

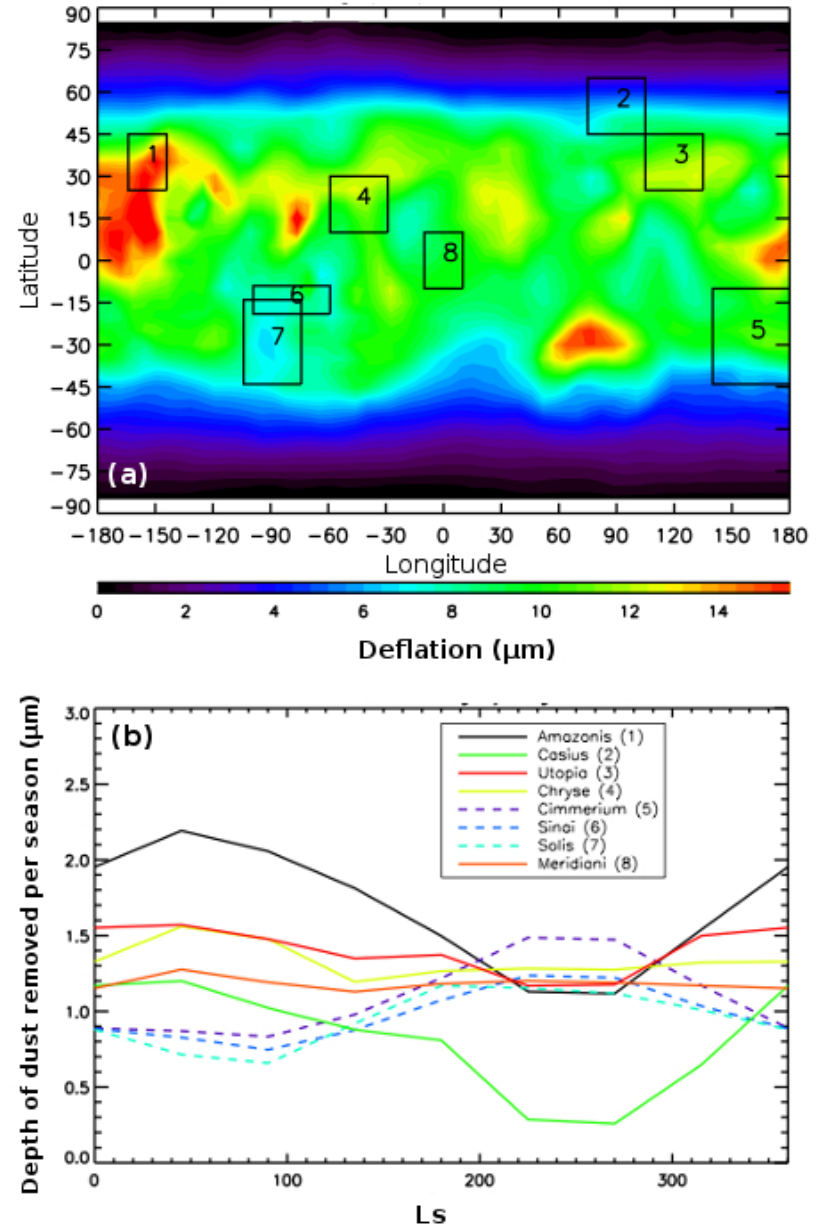

Fig. 17 (a) Annual surface dust removal by dust devils on Mars as obtained by Kahre et al. (2006) using a threshold-independent dust devil parameterization. Boxes indicate areas surveyed by Fisher et al. (2005). (b) Seasonal dust removal by dust devils for the areas labeled in (a). Reprinted from Kahre et al. (2006), with permission from John Wiley and Sons.

ground dust loading too in these models (requiring further tuning of the dust devil contribution), the grid spacings used in most current global Mars models mean that many small-scale winds are not resolved. Thus it should be noted that the dust devil parameterization may be used to account for dust in the global budget that is really lifted by other sub-grid scale circulation features (e.g. small-scale slope or ice cap edge winds, or convective gusts) which are 
also not represented explicitly, and hence the dust lifted by such a scheme is an over-estimate of the true dust devil lifting if the total budget is correct. It is likely, however, that as Mars models increase in resolution the dust lifting generated by the saltation bombardment scheme will become increasingly realistic, thus leading to a more accurate estimate of dust devil lifting.

Kahre et al. (2006) estimated that dust devils contribute $50 \%$ of the annual atmospheric dust loading during a year without a global-scale dust storm. This is broadly consistent with figures published in Newman et al. (2005) for the present orbital epoch, who show the total amount of dust lifted from the surface by each mechanism. Also, this fits with the observational estimates on the annual dust devil flux mentioned in Section 4.1 (Whelley and Greeley 2008; Cantor et al. 2006). The dust devil lifting has peak dust depletion values which are roughly a factor of two less than the peaks of saltation lifting from explicit wind stress, but the dust devil lifting occurs over a wider area of the planet and is broadly comparable in total effect.

\subsubsection{Scaling dust fluxes based on Large Eddy Simulations of a dust devil population (Earth)}

On Earth, particular attention has been given to the development of schemes that represent dust emission during large-scale dust events such as dust storms. Dust emission in regional and global models has typically been incorporated as a single dust emission parameterization, irrespective of the meteorological processes leading to dust emission. Dust emission schemes are usually based on the process of saltation bombardment (e.g. Shao et al. 1993; Marticorena and Bergametti 1995; Shao 2004; Kok et al. 2014). Dust emission estimates in most models for Earth are thus based on friction velocity, $u_{*}$, obtained from a surface layer parameterization, and on a threshold friction velocity, $u_{* t}$, for saltation (see Chap. 10 [Neakrase et al., 2016]). The dust emission flux is thus only dependent on bulk properties of the atmosphere and of the surface. Progress has been achieved by accounting for subgrid-scale winds in regional and global simulations induced by, for example, dry and/or moist convection (Lunt and Valdes 2002; Cakmur et al. 2004; Takemi et al. 2006; Pantillon et al. 2015). These studies assess the change in modeled dust emissions due to small- and meso-scale winds, but it is not possible to separately assess sediment transport from dust devils alone from such results. However, although convective vortices 
are small-scale events, they can be frequent and widespread and thus may lift sediment amounts that accumulate to a significant portion of the dust budget, especially in areas where dust devil occurrence frequency is higher than on global average.

This motivated Klose and Shao (2012) and Klose et al. (2014) to develop a parameterization scheme that would represent the direct aerodynamic entrainment of dust by atmospheric turbulence in the absence of strong mean winds leading to systematic saltation. The scheme was shown to be able to simulate the dust lifting in dust devils in the framework of LES (Klose and Shao 2013, 2016). Klose and Shao (2016) analyzed the dust emission generated by dust devils occurring in their LES and proposed a method to estimate dust devil dust flux per unit area and unit time, $\tilde{F}$, as

$$
\tilde{F}=n\left\langle M_{D D}\right\rangle
$$

where $\left\langle M_{D D}\right\rangle[\mathrm{kg}]$ is the ensemble average of the dust mass transported by individual dust devils. By using $\left\langle M_{D D}\right\rangle$, the use of 'typical' values for dust devil size, duration, and intensity can be avoided (see Section 4.2.1). From their simulations, Klose and Shao (2016) obtain

$$
\left\langle M_{D D}\right\rangle= \begin{cases}0.17 \times \exp (8.15 R i)+0.015 & \text { for } R i<0 \\ 0 & \text { otherwise. }\end{cases}
$$

Together with Equation (5), Equations (11) and (12) allow to readily estimate dust devil dust transport in global models based on Richardson number. This enables the separate estimation of dust devil dust transport, for the first time using surface dust emission fluxes.

It must be noted that Equation (11) was obtained based on idealized simulations, i.e. using a homogeneous land-surface, and effects of vegetation cover, soil moisture, or soil type are not yet included. To account for changes in vegetation cover, a preliminary correction for $N$ was applied (Section 3.3.2). The estimated dust fluxes do not account for variations in soil type at the moment, but are based on calculations for a loam soil. Further LES runs would be required to quantify the effects of changes in vegetation cover and soil type on $\left\langle M_{D D}\right\rangle$ and incorporate them into Eq. (12). This is feasible as the LES model and the dust emission scheme used by Klose and Shao (2016) are capable of accounting for flow changes due to vegetation or other roughness elements (Shao et al. 2013) and changes in dust emissions due to different soil types. 

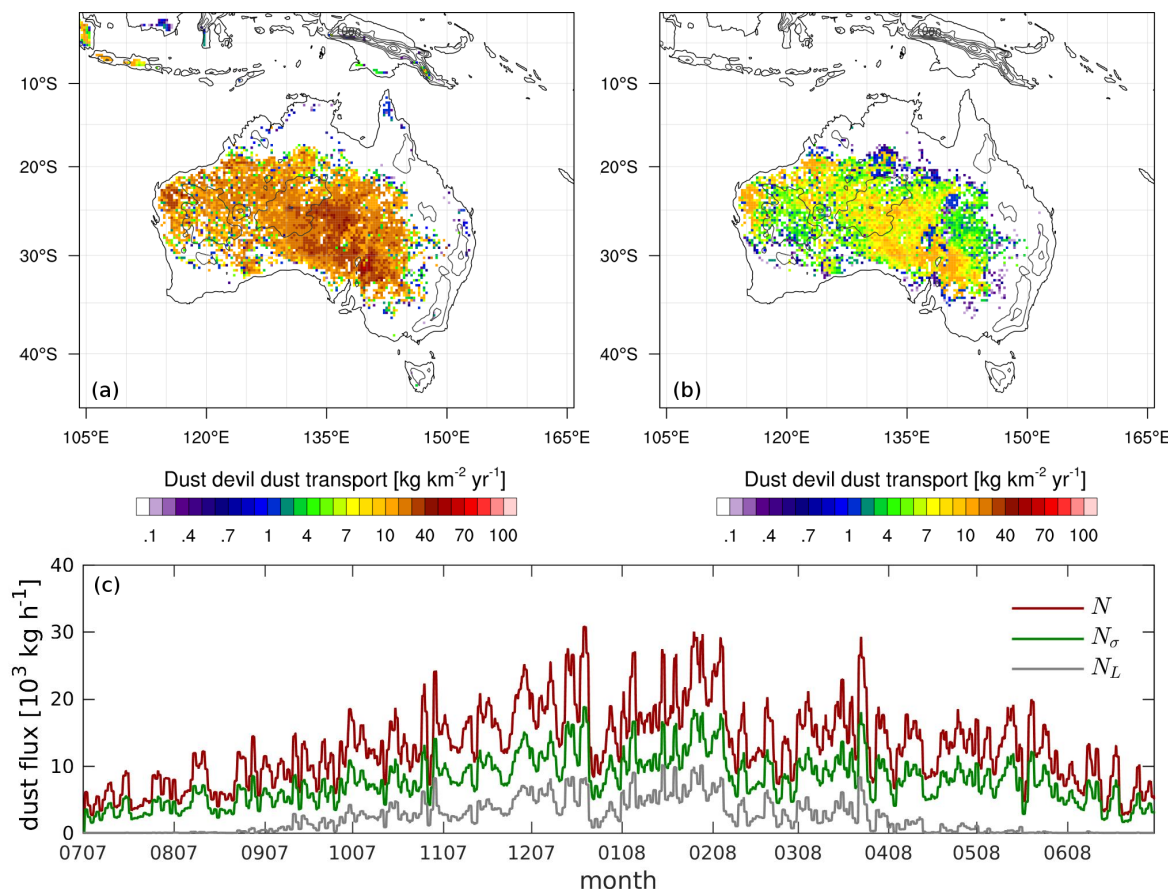

Fig. 18 (a) Estimate of the total dust transport by dust devils in Australia from July 2007 to July 2008 based on Equations (11), (12), and $N_{\sigma}$ (Eq. (5) with correction for vegetation cover); (b) as (a), but using $N_{L}$ (Eq. (5) with vegetation correction and lapse rate criterion). (c) Time series of total dust devil dust transport in the simulation domain. Lines show 24-h running means of hourly dust tranport estimated based on $N$ (Eq. (5), red), $N_{\sigma}$ (green), and $N_{L}$ (gray).

Applying Equations (5), (11), and (12) to the model results of Klose (2014) for Australia (see Section 3.3.2) yielded an estimate of the contribution of dust devils to the Australian dust budget. In dependence on the number of dust devils, the amount of dust transported by dust devils varies with season. Based on $N_{\sigma}$ as the number of dust devils, the largest fluxes occured in southern hemispheric summer with $1-3 \mathrm{~kg} \mathrm{~km}^{-2} \mathrm{mon}^{-1}$ in wide areas of central Australia, and up to about $10 \mathrm{~kg} \mathrm{~km}^{-2} \mathrm{mon}^{-1}$ at particular locations. During the year of simulation, totally between 10 and $50 \mathrm{~kg} \mathrm{~km}^{-2} \mathrm{yr}^{-1}$ of dust were transported by dust devils in central Australia based on this approach. Larger values only occured at particular grid points. Almost identical results were obtained if a minimum lapse rate of $1 \mathrm{~K} \mathrm{~m}^{-1}$ was used as additional criterion for the number of dust devils, but smaller fluxes of predominantly $5-30 \mathrm{~kg} \mathrm{~km}^{-2} \mathrm{yr}^{-1}$ were obtained during the simulation year using a minimum lapse rate of $8.5 \mathrm{~K} \mathrm{~m}^{-1}$ 
(Figure 18b). This estimate is smaller than that obtained by Jemmett-Smith et al. (2015), which shows values for dust devil dust transport in the category of $0-2 \times 10^{3} \mathrm{~kg} \mathrm{~km}^{-2} \mathrm{yr}^{-1}$ for most regions of Australia, but up to $\sim 20 \times 10^{3} \mathrm{~kg} \mathrm{~km}^{-2} \mathrm{yr}^{-1}$ at particular locations.

Figure 18c shows time series of domain integrated dust transport by dust devils during the investigation period. While dust fluxes of up to about $5 \times$ $10^{3} \mathrm{~kg} \mathrm{~h}^{-1}$ were determined for the winter months June and July when using $N_{\sigma}$, no significant dust devil dust transport is estimated based on $N_{L}$ (minimum lapse rate of $8.5 \mathrm{~K} \mathrm{~m}^{-1}$ required). Dust fluxes remain below 20 and $10 \times 10^{3} \mathrm{~kg} \mathrm{~h}^{-1}$ for $N_{\sigma}$ and $N_{L}$, respectively, during the whole year. Without correction for vegetation cover, i.e. using $N$ as in Eq. (5), dust fluxes can exceed $10 \times 10^{3} \mathrm{~kg} \mathrm{~h}^{-1}$ in winter and reach maxima of about $27 \times 10^{3} \mathrm{~kg} \mathrm{~h}^{-1}$ in summer.

In total, a dust mass of about $0.11,0.07$, or $0.03 \times 10^{9} \mathrm{~kg} \mathrm{yr}^{-1}$ was lifted by dust devils when using $N, N_{\sigma}$, or $N_{L}$ as the number of dust devils. Estimates of total annual dust emissions for Australia vary largely (Huneeus et al. 2011; Shao et al. 2011). For particles with diameters of up to $20 \mu \mathrm{m}$, estimates range from 14.9 to $106 \times 10^{9} \mathrm{~kg} \mathrm{yr}^{-1}$ with an average of $59 \times 10^{9} \mathrm{~kg} \mathrm{yr}^{-1}$ (Tanaka and Chiba 2006; Huneeus et al. 2011). Compared to this, the dust devil contribution to the Australian dust budget would be very small $(<1 \%)$. It must be noted, however, that the dust emission fluxes obtained by Klose and Shao (2016) are smaller or on the lower edge of those obtained in laboratory or field (Neakrase and Greeley 2010b; Metzger et al. 2011) as only aerodynamic entrainment is accounted for in the model and intermittent saltation is not yet included. Additionally, large parts of Australia have soil types with a larger fraction of dust-size particles than loam, e.g. sandy loam or clay, thus leading to larger dust emission fluxes. An increase in dust flux by $1-2$ orders of magnitude to be closer to the values estimated by Renno et al. (1998) and Metzger et al. (2011), would yield a total contribution of only between 0.3-19\%. On the contrary, surface crusting especially in clay-rich areas might again reduce dust emission and is not yet accounted for in any of the estimates given in this paper. Not least, the choice of the reference value (here $59 \times 10^{9} \mathrm{~kg} \mathrm{yr}^{-1}$ ) is naturally pivotal to the resulting dust devil contribution. 


\section{Impact on local, regional and global scales}

The frequency of dust devils varies strongly from region to region and from season to season both on Earth and Mars. Dust devils are most significant on local and regional scales, less so on global scales, as has been shown in the previous sections. Nevertheless, the impact of dust devils on all spatial scales in the Earth system and in the martian environment can be manifold. The effects extend from property/instrument damage on local scales, to air quality on regional scales, to climate feedbacks on global scales. Despite their relatively small extent and short duration, their frequency of occurrence in particular areas make them a fundamental mechanisms for dust uplift with important impacts on daily life on Earth as well as on the climate and environment of Earth and Mars.

\subsection{Local scale: Incidents caused by dust devils}

At a local level, the winds associated with dust devils can be disruptive to picnics, sports etc., although occasionally can cause damage to light structures and even occasional deaths. These effects are due to the high winds within dust devils and are summarized briefly in Chap. 1 (Lorenz et al., 2016). The dust lifted by dust devils can present health and more general air quality issues, as is discussed at the regional level in Section 5.2. Local dust-lifting can also have a local impact on visibility: this may have been a factor in at least one vehicular fatality attributed to a dust devil (a South African astronomer - see Lorenz 2013) and the loss of pilot situational awareness in a hovering helicopter due to the dust in a dust devil was a stated factor in the crash of that aircraft (Lorenz and Myers 2005). On Mars, Spirit rover measurements of background dust opacity show $4-6$ times larger opacities during the dust devil season compared to other seasons at Gusev crater on Mars (Greeley et al. 2010). The large number of dust devils on Mars during southern hemispheric spring and summer compared to other seasons suggests a strong contribution of dust devils to the generation of local dust haze at that time of year. However, a larger dust load decreases dust devil activity due to its negative feedback on surface sensible heat flux. In particular, regional-scale dust storms can suddenly increase dust opacity and thus shut down dust devil activity (Greeley et al. 2010). 
The local impact of dust transport by dust devils is not always negative, however. Dust devils appear to be responsible for the 'dust-clearing events' noted on the Mars Exploration Rover Spirit (Lorenz and Reiss 2015) which restored the electrical output of its solar panels. Steady accumulation of dust on the panels leads to a progressive drop in power, which would eventually end the mission, but at the start of dust devil season, dust was suddenly removed and power levels improved, allowing the mission to operate much longer than originally planned.

5.2 Regional scale: Dust devil feedback to the surface and the atmosphere

\subsubsection{Earth}

As shown in this paper, the dust devil contribution to the continental or global dust budgets on Earth seems to be small. However, dust devils can be a major dust event type on regional scales depending on the season, such as in the Eldorado (Nevada) or Avra Valleys (Arizona), USA (Sinclair 1969; Metzger et al. 2011), in parts of the Atacama and Sechura deserts in South America (Kurgansky et al. 2011; Hesse 2012; Reiss et al. 2013; Jemmett-Smith et al. 2015), or at locations in southeastern Australia (Oke et al. 2007b; Klose and Shao 2016). As a large part of the dust lifting in dust devils occurs through aerodynamic entrainment, dust devils can still cause emissions from soil surfaces which contain little or no particles in the saltation size range. This may for example be the case on playas, where the surface is crusted but may be covered by a thin dust layer, or on loosely packed silt- and/or clay-rich soils, such as on some cultivated agricultural fields. On such surfaces, dust devils may become a significant emission process.

In regions that favor dust devil development, the frequent occurrence of dust devils leads to a persistent removal of the top-soil layer. Although dust devils do not travel long distances, the vertical mixing in the convective boundary layer is intense. As a consequence, suspended particles are likely transported farther than the location of dust devil cessastion might suggest, thus potentially leading to a net soil transport away from the typical formation regions of dust devils. As discussed by Oke et al. (2007a), this might lead to local topographic changes between non- or weakly vegetated areas where dust devils occur frequently, and strongly vegetated areas, thus potentially affecting 
the hydrological cycle in the area. Not least, the loss of top-soil particles is associated with a loss of soil nutrients, minerals, and carbon, thus impacting on soil productivity (e.g. Sterk et al. 1996; Webb et al. 2013). The degree to which these effects occur in a particular area and the feedbacks they have on dust devil formation, i.e. smaller availability of loose soil particles or changes in water flow, would need to be investigated on a case by case basis.

The strong vertical mixing of particles entrained by dust devils also has an effect on air quality. Due to the small terminal velocities of particles in the lower dust-size range, e.g. below $10 \mu \mathrm{m}$ in diameter, these particles, once entrained, have long atmospheric residence times (Shao 2008). The frequent occurrence of dust devils in a particular area might thus lead to a significant increase of suspended particulate matter (PM) in the atmosphere. Effects are reduced visibilities (not for very low PM levels), changes in atmospheric thermodynamic properties through radiative interactions, increased availability of cloud condensation and ice nuclei, and damage to human and animal health through inhalation of pathogens or chemical contaminants transported with dust particles (Rosenfeld et al. 2001; Kellogg and Griffin 2006; Boucher et al. 2013; Miller et al. 2014).

\subsubsection{Mars}

When dust devils inject dust to great heights, through the deep planetary boundary layer (Petrosyan et al. 2011) and into the free atmosphere, it can be advected to much greater heights by the global circulation and reach 80 $\mathrm{km}$ altitude or more. This dust is rapidly mixed and thus is indistinguishable from that injected by any other source. Dust absorption is a major source of internal heating in the martian atmosphere and a primary component of the radiative-dynamic feedback and hence interannual variability (e.g. Read and Lewis 2004).

On a smaller scale, the direct radiative effects of dust associated with dust devil circulations have yet to be studied in great detail. Fuerstenau (2006) suggested that dust devils might be larger on Mars than on Earth as a result of solar heating via dust absorption. Internally-heated parcels of air would become more positively buoyant as a result of the warming and so ascend to greater heights, enhancing the dust devil. A similar radiative feedback that could lead to convective instability within larger, but still local dust storms 
has been demonstrated in a mesoscale model (Spiga et al. 2013). These calculations were, however, still for circulations on much larger scales than a typical individual dust devil that would have a lower dust load. Whether or not an individual dust devil would be prone to radiative feedback is yet to be investigated. The coupled modeling needed to establish this has not been performed for Mars, although there has been some initial progress with Monte Carlo simulations of radiative transfer within idealized dust devil-like cylindrical dust distributions (Mason et al. 2013).

It is clear that, although the amount of dust lifted by dust devils on Mars is difficult to quantify, the dust that is lifted can have a profound impact on atmospheric circulations through radiative feedback. It is less clear that dust devil lifting can have a profound effect upon the surface of the planet in turn. The most obvious change seen is the presence of graffiti-like dust devil tracks that can be seen from orbit (see Figure 10). The tracks appear dark (sometimes bright) compared to their surroundings (see Chap. 4 [Reiss et al., 2016]). This is because the bedrock itself is darker than the fine layer of dust which is deposited over it, and which tends to settle slowly from the atmosphere so forming a smooth surface with slightly higher albedo. Where the dust devils pass they lift some of the fine material and also act to redistribute grains of various sizes, changing the surface roughness and leaving a darker track. It may not be the case that all the fine dust is removed, leaving clean rock, but rather that the distribution of granular particle sizes is mixed and altered (for the different formation processes, see Chap. 4 [Reiss et al., 2016] and Reiss et al. 2010, 2011, 2013). Verba et al. (2010) show some more examples of dust devil tracks on Mars. Michaels (2006) provided an example simulation of a dust devil generating a track (see Chap. 7 [Spiga et al., 2016]).

Although dust devils clearly change the albedo of the martian surface on small scales (the tracks are of the order of magnitude $10 \mathrm{~m}$ wide and may be coherent over distances of kilometers or spiral and fade rapidly) there is presently little evidence that they change the landscape in a consistent way on longer timescales, for example by preferentially stripping dust from certain regions of the planet on an annual-mean basis. There are very many dust devils that occur over large regions of the surface. Dust is lifted from a variety of sites in an apparently stochastic pattern and eventually settles back down from the atmosphere. If dust devils were equally common everywhere, then such a 
process would produce no long term trend in dust distribution. Dust devils do, however, seem to be less common in certain localities, such as Gale Crater (Moores et al. 2015). If dust devils are a major component of the equatorial dust lifting and are not counter-balanced by enhancements in other small-scale effects, such as slope winds, then such regions should preferentially acquire fine dust over long timescales. Although relatively small masses of surface material are moved by each dust devil, any consistent bias in lifting might make significant changes to the size-distribution of dust in the upper layers of the regolith. Any consistent change to surface albedo over larger areas affects the radiative budget at the surface and so impacts on the martian circulation and climate in turn.

5.3 Global scale: interactions with climate and environment

\subsubsection{Earth}

For Earth, only two studies (Koch and Renno 2005; Jemmett-Smith et al. 2015) have attempted to upscale dust devil dust emissions to global scales to get an estimate of their contribution to the total emissions. Best estimates given by these studies vary by one order of magnitude, illustrating the substantial multiplicative uncertainties related particularly to dust flux and source area. Koch and Renno (2005) estimated a contribution of dust devils to the global dust budget of $26 \% \pm 18 \%$ based on a global annual dust emission of $2150 \times 10^{9} \mathrm{~kg}$ (IPCC 2001). Recent results by Jemmett-Smith et al. (2015) estimated that dust devils contribute $\sim 3 \%$ (uncertainty 1 to $25 \%$ ) to the total mineral dust budget on Earth, when assuming an annual global dust emission of $2000 \times 10^{9} \mathrm{~kg}$ (Griggs and Noguer 2002; Shao et al. 2011; Huneeus et al. 2011). In addition, if the dust devil flux values given by Metzger et al. (2011) were applied to the results by Koch and Renno (2005) and Jemmett-Smith et al. (2015), both would give dust devil contributions of less than $1 \%$ to the total mineral dust budget on Earth.

The continental-scale estimate for Australia shown in Section 4.2.3 seems to support that dust devils are of little importance from the perspective of global-scale dust emissions. With an estimated contribution of dust devils of $0.03-0.19 \%$ to the Australian dust budget, the dust transported by dust devils is smaller than one would think given the high frequency of dust devil reports 
on the continent (Fig. 9). For the USA, Gillette and Sinclair (1990) found that dust devils accounted for about $66 \%$ of the total lifted dust mass on the continent based on aircraft measurements conducted near Tucson, Arizona. The estimate obtained by Gillette and Sinclair (1990) is grounded on general assumptions on the relative magnitude of the dust flux in areas other than the test area that are based on climate and vegetation. The estimate does thus likely contain large uncertainty. In addition, no seasonal variation has been considered, so the percentage resulting from a dust devil cencus conducted during the dust devil season is probably an overestimation. However, the study by Gillette and Sinclair (1990) was the first that aimed to upscale dust devil measurements to a continental scale. Overall, the later results suggest that dust devils are unlikely to be a big player in the global mineral dust cycle on Earth.

\subsubsection{Mars}

As has been noted throughout this paper, observations of martian dust devils are incomplete, with significant detections at only a handful of lander sites for limited periods (see Section 3.2) and through orbital imaging of the largest dust devils and their surface tracks. Any quantitative estimates of global impacts are therefore reliant to some extent on extrapolation and modeling, with considerable uncertainties remaining in both.

The impact of dust devils on the global atmospheric dust loading is still highly uncertain. As noted in Section 4.2.2, many Mars models employ specific parameterizations for dust devils (e.g. Newman et al. 2002a,b; Basu et al. 2004; Kahre et al. 2006; Mulholland et al. 2013; Newman and Richardson 2015). In these models the dust devils parameterization contributes up to one half of the total annual dust budget (Newman et al. 2005; Kahre et al. 2006). Such lifting tends to peak around both solstices in the summer hemisphere, though with greater lifting in the southern hemisphere during summer. However, the spatial and temporal variation in dust devil lifting is relatively smooth, and there is at least weak dust devil lifting predicted over most of the planet at most times of year. This is in direct contrast to lifting from explicit nearsurface wind stress schemes, which tend to peak in restricted locations and times (particularly during the dust storm season, solar longitude $L_{\mathrm{s}} \sim 180^{\circ}-$ 
$360^{\circ}$ ), although they may lift several times more dust at these peak locations (e.g. Cantor et al. 2001; Cantor 2007; Wang and Richardson 2015).

Although dust may remain suspended for many tens of days in the martian atmosphere, even global dust events decay more rapidly than the seasonal timescale. The result is that the dust devil parameterization is required to support the background loading of dust in the model, as observed throughout the year, particularly in the northern hemisphere summer $\left(L_{\mathrm{s}}=90^{\circ}-180^{\circ}\right)$, when dust loadings are lower, but still significant and repeatable (Smith 2004; Montabone et al. 2015). Section 4.2.2 has already questioned whether the total dust lifting that is accounted for by the dust devil parameterization in a model is truly the result of lifting by dust devils alone or may be used to account for other small scale lifting, from mesoscale and microscale winds, that is not represented in the model. The parameterization is likely tuned to account for all missing processes. On the other hand, such schemes have been shown to reproduce broadly the observed seasonal behavior of dust devil activity in both the northern and southern hemispheres (Fisher et al. 2005; Kahre et al. 2006) and so are not totally misleading.

Newman et al. (2002b) show that a dust devil parameterization, in contrast to the near-surface wind stress parameterization, typically has a negative feedback: a clearer atmosphere results in a greater atmosphere-surface thermal contrast and so increases dust devil activity and vice versa. Thus the dust devil lifting scheme will tend to prevent the model atmosphere from reaching unrealistically low dust loadings, even in northern hemisphere summer when winds are relatively weak on average. Even at these lower levels (typically visible total opacities of $0.1-0.2$ ), atmospheric dust still plays a major role in the martian radiation budget.

\section{Conclusions}

This paper provides a review of dust devil dust transport on Earth and Mars based on state-of-the-art methods in laboratory-based and field studies, remote sensing, and modeling. Technological advances have led to significant advances during the recent years, providing means for further investigations of dust devils. 
Laboratory studies on dust devils (Neakrase et al. 2006; Neakrase and Greeley 2010b,a) allowed for the detailed investigation of individual vortices and thus provided insights into dust devil dynamics. Further developed instruments, increasing data storage capacity, and higher-resolved images led to more comprehensive (in situ and remote) field observations of dust devils (e.g. Metzger et al. 2011; Mason et al. 2014; Whelley and Greeley 2008; Lorenz and Jackson 2015; Reiss and Lorenz 2015). High-performance computing environments allow for the use of high-resolution large-eddy simulations - with and without coupled dust emission schemes - to study the characteristics of individual dust devils. New methods to estimate dust devil occurrence frequency and dust devil dust transport (Jemmett-Smith et al. 2015; Klose and Shao 2016) yield the opportunity to study the effect of dust devils on a significantly higher spatio-temporal resolution, thus hopefully leading to more accurate results. In global models, the use of dust devil parameterizations enabled the reproduction of the martian background annual dust cycle (e.g. Newman et al. 2002a; Kahre et al. 2006).

Naturally, the overlap in methods used for Earth and Mars is small in the field of direct observations. On Earth, in situ observations are more easy, than on Mars, as the areas are more accessible. Owing to the stochastic nature of dust devils, their in situ measurement remains challenging, however. On Mars, the observations have a stronger focus on dust devil detections from imagery, which may bias them more toward larger events. However, while the use of imagery data for dust devil analyses is common practice for Mars, it is not as well established for Earth. This leads to more comprehensive observational datasets on the spatial distribution of dust devils for Mars, while datasets for Earth focus more on the properties of individual dust devils, which vary significantly. Consequently, it is important to ensure that general statements regarding dust devil properties are based on a sufficiently large dust devil population (see also Chap. 8 [Lorenz and Jackson, 2016]). If such care is not taken, then conclusions - on topics such as the contribution of dust devils to the total dust budget - may not be representative.

Large-eddy simulation models can be applied similarly effective for Earth as for Mars, although domain extents need to be larger on Mars due to a deeper planetary boundary layer. Hence, more computing power and memory would be required if simulations were to be conducted at a similar spatial reso- 
lution. Comparison of the model results to observations is again more difficult for Mars due to limited data availability. This complicates the application of dust emission schemes developed and calibrated for Earth to the simulation of martian dust devils. For both, Earth and Mars, large-eddy simulation is usually applied in an idealized setup. The investigation of the effects of non-ideal conditions on dust devil properties, such as surface heterogeneity, vegetation cover, etc., requires additional modeling efforts in the future. Some of the models available to date already provide the necessary framework to conduct such modeling studies.

Different methods are used in terrestrial and global Mars models. While the focus of dust modeling on Earth has until recently almost exclusively been on large-scale dust events such as dust storms, separate parameterization schemes for dust devils have existed for a while for use in martian global models. This originates from the important role dust devils play in the martian climate, whereas they likely have smaller effects on the terrestrial climate. The dust devil parameterizations in models account for roughly half the total martian dust lifting, although it is arguable whether all this lifting is truly a result of dust devils or other small lifting events not captured in the models. These parameterizations are constrained only to reproduce the global atmospheric dust budget over annual timescales, acting in combination with saltation lifting by explicitly resolved winds. Although there is some broad agreement in terms of the time of day and year for peak lifting between model lifting schemes and observations of dust devil occurrence, the details of peak location differ and the actual lifted dust flux can only be roughly estimated.

The results of this paper suggest that dust devils on Earth are more likely to have local and regional effects on air quality and potentially on geomorphology, than global effects on climate. These potential environmental impacts of dust devils have not yet been quantified, however. On Mars, the radiative feedback of dust haze generated by dust devils may be significant on regional and possibly also on global scales, although uncertainties exist concerning the accuracy of estimated dust devil contributions to global dust aerosol. Due to the wide distribution of regions prone to dust devil occurrence, a strong effect on martian geomorphology appears unlikely. However, areas that exhibit persistent large numbers of dust devils may experience a significant sorting of top-soil particles both on Earth and Mars. 
The methods and tools summarized in this paper provide the necessary means to quantify dust devil sediment transport on different spatial scales and for different locations, thus enabling the investigation of the effect of dust devils on local climate and geomorphology. It is now possible to conduct highresolution studies of dust devils for focus areas with regional models, where in-situ field observations and remote sensing data can be compared to model outputs to gain knowledge of the meteorological conditions controlling their occurrence, and to further improve their representation in model parameterizations. At the moment, the discrepancies between individual research outcomes are large. Understanding the benefits and limitations of the different approaches is essential to interpret the results. A closer linkage between research disciplines, approaches, and study areas would help to relate individual research outcomes and be highly beneficial for the study of dust devils in the future, as would more interaction between those working on Earth and Mars studies, especially in remote sensing an modeling.

\section{Acknowledgments}

We wish to thank Luca Montabone, one anonymous reviewer, and two editors for their careful review and valuable comments, and Bruce Cantor for his permission to reuse Figure 4 of Cantor et al. (2006) in this paper. Bradley Jemmett-Smith and Peter Knippertz would like to acknowledge funding from the European Research Council Grant 257543 "Desert Storms “. Ralph Lorenz acknowledges the support of NASA Mars Fundamental Research Program grant NNX12AI04G. Not least, we are grateful to the International Space Science Institute (ISSI), Bern, Switzerland, and to the conveners for organizing the workshop "Dust devils on Mars and Earth" (www.issibern.ch/ workshops/dustdevils/).

\section{References}

A. Ansmann, M. Tesche, P. Knippertz, E. Bierwirth, D. Althausen, D. Müller, O. Schulz, Vertical profiling of convective dust plumes in southern Morocco during SAMUM. Tellus B 61(1), 340-353 (2009)

R.A. Bagnold, The Physics of Blown Sand and Desert Dunes (Methuen, London, 1941), p. 265 
M. Balme, R. Greeley, Dust devils on Earth and Mars. Rev. Geophys. 44 (2006). doi:10.1029/2005RG000188

M. Balme, A. Hagermann, Particle lifting at the soil-air interface by atmospheric pressure excursions in dust devils. Geophys. Res. Lett. 33(L19S01), 3-7 (2006). doi:10.1029/2006GL026819

M.R. Balme, P.L. Whelley, R. Greeley, Mars: Dust devil track survey in Argyre Planitia and Hellas Basin. J. Geophys. Res. 108(E8) (2003). 5086. doi:10.1029/2003JE002096. http://dx.doi.org/10.1029/2003JE002096

M. Bangert, A. Nenes, B. Vogel, D. Barahona, V.A. Karydis, P. Kumar, C. Kottmeier, U. Blahak, Saharan dust event impacts on cloud formation and radiation over Western Europe. Atmos. Chem. Phys. 12, 4045-4063 (2012). doi:0.5194/acp-12-4045-2012

S. Basu, M.I. Richardson, R.J. Wilson, Simulation of the Martian dust cycle with the GFDL Mars GCM. Journal of Geophysical Research: Planets 109(E11) (2004). E11006. doi:10.1029/2004JE002243. http://dx.doi.org/10.1029/2004JE002243

O. Boucher, D. Randall, P. Artaxo, C. Bretherton, G. Feingold, P. Forster, V.-M. Kerminen, Y. Kondo, H. Liao, U. Lohmann, P. Rasch, S.K. Satheesh, S. Sherwood, B. Stevens, X.Y. Zhang, Clouds and Aerosols, in Climate Change 2013: The Physical Science Basis. Contribution of Working Group I to the Fifth Assessment Report of the Intergovernmental Panel on Climate Change, ed. by T.F. Stocker, D. Quin, G.-K. Plattner, M. Tignor, S.K. Allen, J. Boschung, A. Nauels, Y. Xia, V. Bex, P.M. Midgley (Cambridge University Press, Cambridge, United Kingdom and New York, NY, USA, 2013)

C.S. Bristow, K.A. Hudson-Edwards, A. Chappell, Fertilizing the Amazon and equatorial Atlantic with West African dust. Geophys. Res. Lett. 37 (2010). doi:10.1029/2010GL043486

R.V. Cakmur, R.L. Miller, O. Torres, Incorporating the effect of small-scale circulations upon dust emission in an atmospheric general circulation model. J. Geophys. Res. Atmos. 109(D7) (2004). D07201. doi:10.1029/2003JD004067

B.A. Cantor, K.M. Kanak, K.S. Edgett, Mars Orbiter Camera observations of Martian dust devils and their tracks (September 1997 to January 2006) and evaluation of theoretical vortex models. J. Geophys. Res. 111(E12002) (2006). doi:10.1029/2006JE002700

B.A. Cantor, MOC observations of the 2001 Mars planet-encircling dust storm. Icarus 186(1), 60-96 (2007). doi:10.1016/j.icarus.2006.08.019

B.A. Cantor, P.B. James, M. Caplinger, M.J. Wolff, Martian dust storms: 1999 Mars Orbiter Camera observations. J. Geophys. Res. 106(E10), 23653-23687 (2001). doi:10.1029/2000JE001310

J.J. Carroll, J.A. Ryan, Atmospheric vorticity and dust devil rotation. J. Geophys. Res. 75(27), 5179-5184 (1970). doi:10.1029/JC075i027p05179

D.S. Choi, C.M. Dundas, Measurements of Martian dust devil winds with HiRISE. Geophys. Res. Lett. 38(24) (2011). L24206. doi:10.1029/2011GL049806. http://dx.doi.org/10. 1029/2011GL049806

S.M. Cowie, P. Knippertz, J.H. Marsham, Are vegetation-related roughness changes the cause of the recent decrease in dust emission from the Sahel? Geophys. Res. Lett. 40(9), 1868-1872 (2013)

C. de Beule, G. Wurm, T. Kelling, M. Küpper, T. Jankowski, J. Teiser, The martian soil as 
a planetary gas pump. Nature Physics 10, 17-20 (2014). doi:10.1038/nphys2821

P. De Deckker, C.I. Munday, J. Brocks, T. OLoingsigh, G.E. Allison, J. Hope, M. Norman, J.-B.W. Stuut, N.J. Tapper, S. van der Kaars, Characterisation of the major dust storm that traversed over eastern australia in september 2009; a multidisciplinary approach. Aeolian Research 15, 133-149 (2014). doi:10.1016/j.aeolia.2014.07.003

J.W. Deardorff, A numerical study of three-dimensional turbulent channel flow at large reynolds numbers. J. Fluid Mech. 41(2), 453-480 (1970). doi:10.1017/S0022112070000691

J. Deardorff, Observed characteristics of the outer layer. Short course on the planetary boundary layer (1978)

P.J. DeMott, K. Sassen, M.R. Poellot, D. Baumgardner, D.C. Rogers, S.D. Brooks, A.J. Prenni, S.M. Kreidenweis, African dust aerosols as atmospheric ice nuclei. GRL 30(14) (2003)

E. Derbyshire, Natural minerogenic dust and human health. AMBIO 36(1), 73-77 (2007). doi:10.1579/0044-7447(2007)36[73:NMDAHH]2.0.CO;2

M.D. Ellehoj, H.P. Gunnlaugsson, P.A. Taylor, H. Kahanp, K.M. Bean, B.A. Cantor, B.T. Gheynani, L. Drube, D. Fisher, A.-M. Harri, C. Holstein-Rathlou, M.T. Lemmon, M.B. Madsen, M.C. Malin, J. Polkko, P.H. Smith, L.K. Tamppari, W. Weng, J. Whiteway, Convective vortices and dust devils at the Phoenix Mars mission landing site. J. Geophys. Res. 115(E4) (2010). doi:10.1029/2009JE003413. http://dx.doi.org/10.1029/ 2009JE003413

L.K. Fenton, R. Lorenz, Dust devil height and spacing with relation to the martian planetary boundary layer thickness. Icarus 260, 246-262 (2015). doi:10.1016/j.icarus.2015.07.028

F. Ferri, P.H. Smith, M. Lemmon, N.O. Renn, Dust devils as observed by Mars Pathfinder. Journal of Geophysical Research: Planets 108(E12) (2003). doi:10.1029/2000JE001421. http://dx.doi.org/10.1029/2000JE001421

J.A. Fisher, M.I. Richardson, C.E. Newman, M.A. Szwast, C. Graf, S. Basu, S.P. Ewald, A.D. Toigo, R.J. Wilson, A survey of Martian dust devil activity using Mars Global Surveyor Mars Orbiter Camera images. J. Geophys. Res. 110(E3) (2005). E03004. doi:10.1029/2003JE002165. http://dx.doi.org/10.1029/2003JE002165

S.D. Fuerstenau, Solar heating of suspended particles and the dynamics of Martian dust devils. Geophys. Res. Lett. 33(19) (2006). doi:10.1029/2006GL026798

V.H. Garrison, E.A. Shinn, W.T. Foreman, D.W. Griffin, C.W. Holmes, C.A. Kellogg, M.S. Majewski, L.L. Richardson, K.B. Ritchie, G.W. Smith, African and Asian dust: from desert soils to coral reefs. BioScience 53(5), 469-480 (2003). doi:10.1641/00063568(2003)053[0469:AAADFD]2.0.CO;2

B.T. Gheynani, P.A. Taylor, Large Eddy Simulation of typical dust devil-like vortices in highly convective Martian boundary layers at the Phoenix lander site. Planet. Space Sci. 59, 43-50 (2011). doi:10.1016/j.pss.2010.10.011

D.A. Gillette, P.C. Sinclair, Estimation of suspension of alkaline material by dust devils in the united states. Atmos. Environ. 24(5), 1135-1142 (1990)

P. Ginoux, J.M. Prospero, T.E. Gill, N.C. Hsu, M. Zhao, Global-scale attribution of anthropogenic and natural dust sources and their emission rates based on MODIS Deep Blue aerosol products. Rev. Geophys. 50(3) (2012) 
R. Greeley, J.D. Iversen, Wind as a geological process on Earth, Mars, Venus and Titan (Cambridge University Press, New York, 1985), p. 333

R. Greeley, M.R. Balme, J.D. Iversen, S. Metzger, R. Mickelson, J. Phoreman, B. White, Martian dust devils: Laboratory simulations of particle threshold. J. Geophys. Res. 108(E5), 5041 (2003)

R. Greeley, P.L. Whelley, R.E. Arvidson, N.A. Cabrol, D.J. Foley, B.J. Franklin, P.G. Geissler, M.P. Golombek, R.O. Kuzmin, G.A. Landis, M.T. Lemmon, L.D.V. Neakrase, S.W. Squyres, S.D. Thompson, Active dust devils in Gusev crater, Mars: Observations from the Mars Exploration Rover Spirit. J. Geophys. Res. 111(E12) (2006). E12S09. doi:10.1029/2006JE002743. http://dx.doi.org/10.1029/2006JE002743

R. Greeley, D.A. Waller, N.A. Cabrol, G.A. Landis, M.T. Lemmon, L.D.V. Neakrase, M. Pendleton Hoffer, S.D. Thompson, P.L. Whelley, Gusev Crater, Mars: Observations of three dust devil seasons. Journal of Geophysical Research E: Planets 115(9), 1-18 (2010). doi:10.1029/2010JE003608

D.J. Griggs, M. Noguer, Climate Change 2001: The Scientific Basis. Contribution of Working Group I to the Third Assessment Report of the Intergovernmental Panel on Climate Change. Weather 57(8), 267-269 (2002)

Z. Gu, J. Qiu, Y. Zhao, Y. Li, Simulation of terrestrial dust devil patterns. Adv. Atmos. Sci. 25(1), 31-42 (2008). doi:10.1007/s00376-008-0031-7

G. Hess, K. Spillane, Characteristics of dust devils in Australia. J. Appl. Meteor. 29(6), 498-507 (1990)

R. Hesse, Short-lived and long-lived dust devil tracks in the coastal desert of southern Peru. Aeolian Research 5, 101-106 (2012)

N. Huneeus, M. Schulz, Y. Balkanski, J. Griesfeller, J. Prospero, S. Kinne, S. Bauer, O. Boucher, M. Chin, F. Dentener, T. Diehl, R. Easter, D. Fillmore, S. Ghan, P. Ginoux, A. Grini, L. Horowitz, D. Koch, M.C. Krol, W. Landing, X. Liu, N. Mahowald, R. Miller, J.-J. Morcrette, G. Myhre, J. Penner, J. Perlwitz, P. Stier, T. Takemura, C.S. Zender, Global dust model intercomparison in AeroCom phase I. Atmos. Chem. Phys. 11(15), 7781-7816 (2011). doi:10.5194/acp-11-7781-2011. http://www.atmoschem-phys.net/11/7781/2011/

IPCC, Clouds and Aerosols, in Climate Change 2001: The Scientific Basis. Contribution of Working Group I to the Third Assessment Report of the Intergovernmental Panel on Climate Change, ed. by J.T. Houghton, Y. Ding, D.J. Griggs, M. Noguer, P.J. van der Linden, X. Dai, K. Maskell, C.A. Johnson (Cambridge University Press, Cambridge, United Kingdom and New York, NY, USA, 2001), p. 881

K. Isono, On ice-crystal nuclei and other substances found in snow crystals. J. Meteor. 12, 456-462 (1955). doi:10.1016/j.aeolia.2013.11.002

J. Ito, H. Niino, M. Nakanishi, Large eddy simulation on dust suspension in a convective mixed layer. SOLA 6, 133-136 (2010a). doi:10.2151/sola.2010-034 133

J. Ito, R. Tanaka, H. Niino, M. Nakanishi, Large eddy simulation on dust devils in a diurnally-evolving convective mixed layer. J. Meteor. Soc. Japan 88(1), 63-77 (2010b). doi:0.2151/jmsj.2010-105

B.C. Jemmett-Smith, J.H. Marsham, P. Knippertz, C.A. Gilkeson, Quantifying global dust devil occurrence from meteorological analyses. Geophys. Res. Lett. 42(4), 1275-1282 
(2015)

H. Kahanpää, C. Newman, J. Moores, M.-P. Zorzano, J. Martín-Torres, S. Navarro, A. Lepinette, M.T. Lemmon, B. Cantor, P. Valentín-Serrano, A. Ullán, W. Schmidt, Convective vortices and dust devils at the MSL landing site: annual variability. J. Geophys. Res. (2016). submitted

M.A. Kahre, J.R. Murphy, R.M. Haberle, F. Montmessin, J. Schaeffer, Simulating the Martian dust cycle with a finite surface dust reservoir. Geophys. Res. Lett. 32(20) (2005). L20204. doi:10.1029/2005GL023495. http://dx.doi.org/10.1029/2005GL023495

M.A. Kahre, J.R. Murphy, R.M. Haberle, Modeling the Martian dust cycle and surface dust reservoirs with the NASA Ames general circulation model. Journal of Geophysical Research: Planets 111(E6) (2006). E06008. doi:10.1029/2005JE002588. http://dx.doi. org/10.1029/2005JE002588

J.C. Kaimal, J.A. Businger, Case studies of a convective plume and a dust devil. J. Appl. Meteor. 9, 612-620 (1970). doi:10.1175/1520-0450(1970)009;0612:CSOACP ¿2.0.CO;2

K.M. Kanak, Numerical simulation of dust devil-scale vortices. Quarterly Journal of the Royal Meteorological Society 131(607), 1271-1292 (2005). doi:10.1256/qj.03.172

K.M. Kanak, D.K. Lilly, J.T. Snow, The formation of vertical vortices in the convective boundary layer. Quart. J. Roy. Meteor. Soc. 126(569), 2789-2810 (2000). doi:10.1002/qj.49712656910

C.A. Kellogg, D.W. Griffin, Aerobiology and the global transport of desert dust. Trends in Ecology and Evolution 21(11), 638-644 (2006). doi:10.1016/j.tree.2006.07.004

M. Klose, Y. Shao, Stochastic parameterization of dust emission and application to convective atmospheric conditions. Atmos. Chem. Phys. 12(12), 7309-7320 (2012). doi:10.5194/acp-12-7309-2012

M. Klose, Y. Shao, Large-eddy simulation of turbulent dust emission. Aeolian Research 8, 49-58 (2013). doi:10.1016/j.aeolia.2012.10.010

M. Klose, Y. Shao, A numerical study on dust devils with implications to global dust budget estimates. Aeolian Research 22, 47-58 (2016). doi:10.1016/j.aeolia.2016.05.003

M. Klose, Y. Shao, X.L. Li, H.S. Zhang, M. Ishizuka, M. Mikami, J.F. Leys, Further development of a parameterization for convective turbulent dust emission and evaluation based on field observations. J. Geophys. Res. Atmos. 119, 10441-10457 (2014). doi:10.1002/2014JD021688

M.R. Klose, Convective Turbulent Dust Emission: Process, parameterization, and relevance in the Earth system, Dissertation, Universität zu Köln, 2014. http://kups.ub. uni-koeln.de/id/eprint/5826

J. Koch, N.O. Renno, The role of convective plumes and vortices on the global aerosol budget. Geophys. Res. Lett. 32 (2005). doi:10.1029/2005GL023420

J.F. Kok, N.M. Mahowald, G. Fratini, J.A. Gillies, M. Ishizuka, J.F. Leys, M. Mikami, M.-S. Park, S.-U. Park, R.S. Van Pelt, T.M. Zobeck, An improved dust emission model - Part 1: Model description and comparison against measurements. Atmospheric Chemistry and Physics 14(23), 13023-13041 (2014). doi:10.5194/acp-14-13023-2014

J.F. Kok, N.O. Renno, Enhancement of the emission of mineral dust aerosols by electric forces. Geophysical Research Letters 33(19), 2-6 (2006). doi:10.1029/2006GL026284

M. Küpper, G. Wurm, Thermal creep-assisted dust lifting on Mars: Wind tunnel experiments 
for the entrainment threshold velocity. J. Geophys. Res. 120(7), 1346-1356 (2015). 2015JE004848. doi:10.1002/2015JE004848

M.V. Kurgansky, Steady-state properties and statistical distribution of atmospheric dust devils. Geophysical Research Letters 33(19) (2006). doi:10.1029/2006GL026142

M.V. Kurgansky, A. Montecinos, V. Villagran, S.M. Metzger, Micrometeorological conditions for dust-devil occurrence in the Atacama Desert. Boundary-Layer Meteorol. 138(2), 285-298 (2011)

M.T. Lemmon, M.J. Wolff, J.F. Bell III, M.D. Smith, B.A. Cantor, P.H. Smith, Dust aerosol, clouds, and the atmospheric optical depth record over 5 Mars years of the Mars Exploration Rover mission. Icarus 251, 96-111 (2015). Dynamic Mars. doi:10.1016/j.icarus.2014.03.029

H. Lettau, Note on aerodynamic roughness-parameter estimation on the basis of roughness element description. J. Appl. Meteor. 8, 828-832 (1969). doi:10.1175/15200450(1969) $008<0828$ :NOARPE $>2.0 . \mathrm{CO} ; 2$

G.A. Loosmore, J.R. Hunt, Dust resuspension without saltation. J. Geophys. Res. 105(D16), 20663-20671 (2000). doi:10.1029/2000JD900271

R. Lorenz, On the statistical distribution of dust devil diameters. Icarus 215(1), 381-390 (2011). doi:10.1016/j.icarus.2011.06.005

R. Lorenz, The longevity and aspect ratio of dust devils: Effects on detection efficiencies and comparison of landed and orbital imaging at Mars. Icarus 226(1), 964-970 (2013). doi:10.1016/j.icarus.2013.06.031

R.D. Lorenz, Vortex encounter rates with fixed barometer stations: comparison with visual dust devil counts and large-eddy simulations. J. Atmos. Sci. 71, 4461-4472 (2014). doi:10.1175/JAS-D-14-0138.1

R.D. Lorenz, B.K. Jackson, Dust devils and dustless vortices on a desert playa observed with surface pressure and solar flux logging. GeoResJ 5, 1-11 (2015). doi:10.1016/j.grj.2014.11.002

R.D. Lorenz, M.J. Myers, Dust devil hazard to aviation: A review of United States air accident reports. J. Meteorol. 30(298), 178-184 (2005)

R.D. Lorenz, D. Reiss, Solar panel clearing events, dust devil tracks, and in-situ vortex detections on Mars. Icarus 248, 162-164 (2015). doi:10.1016/j.icarus.2014.10.034

R.D. Lorenz, L.D. Neakrase, J.D. Anderson, In-situ measurement of dust devil activity at La Jornada Experimental Range, New Mexico, USA. Aeolian Research, 1-12 (2015). doi:10.1016/j.aeolia.2015.01.012

D.J. Lunt, P.J. Valdes, The modern dust cycle: Comparison of model results with observations and study of sensitivities. Journal of Geophysical Research: Atmospheres 107(D23) (2002). 4669. doi:10.1029/2002JD002316

T. Lyons, U. Nair, I. Foster, Clearing enhances dust devil formation. J. Arid Environ. 72(10), 1918-1928 (2008)

B. Marticorena, G. Bergametti, Modeling the atmospheric dust cycle: 1. Design of a soilderived dust emission scheme. J. Geophys. Res. 100(D8), 16415-16430 (1995)

J.P. Mason, M.R. Patel, S.R. Lewis, Radiative transfer modelling of dust devils. Icarus 223(1), 1-10 (2013). doi:10.1016/j.icarus.2012.11.018

J.P. Mason, M.R. Patel, S.R. Lewis, The retrieval of optical properties from terrestrial dust 
devil vortices. Icarus 231(0), 385-393 (2014). doi:10.1016/j.icarus.2013.12.013

S.M. Metzger, M.R. Balme, M.C. Towner, B.J. Bos, T.J. Ringrose, M.R. Patel, In situ measurements of particle load and transport in dust devils. Icarus 214(2), 766-772 (2011). doi:10.1016/j.icarus.2011.03.013

S.M. Metzger, Dust devils as aeolian transport mechanisms in the southern Nevada and in the Mars Pathfinder landing site, Phd thesis, University of Nevada, 1999

S.M. Metzger, J.R. Carr, J.R. Johnson, T.J. Parker, M.T. Lemmon, Dust devil vortices seen by the Mars Pathfinder Camera. Geophysical Research Letters 26(18), 2781-2784 (1999). doi:10.1029/1999GL008341

T.I. Michaels, Numerical modeling of Mars dust devils: Albedo track generation. Geophys. Res. Lett. 33(19) (2006). doi:10.1029/2006GL026268

T.I. Michaels, S.C.R. Rafkin, Large eddy simulation of atmospheric convection on Mars. Quart. J. Roy. Meteor. Soc. 130, 1251-1274 (2004). doi:10.1256/qj.02.169

D.V. Michelangeli, O.B. Toon, R.M. Haberle, J.B. Pollack, Numerical simulations of the formation and evolution of water ice clouds in the martian atmosphere. Icarus 102(2), 261-285 (1993). doi:http://dx.doi.org/10.1006/icar.1993.1048

R.L. Miller, P. Knippertz, C. Prez Garca-Pando, J.P. Perlwitz, I. Tegen, Impact of Dust Radiative Forcing upon Climate, in Mineral Dust, ed. by P. Knippertz, J.-B.W. Stuut (Springer, Netherlands, 2014), pp. 327-357. ISBN 978-94-017-8977-6. doi:10.1007/97894-017-8978-3_13

L. Montabone, F. Forget, E. Millour, R.J. Wilson, S.R. Lewis, B. Cantor, D. Kass, A. Kleinbhl, M.T. Lemmon, M.D. Smith, M.J. Wolff, Eight-year climatology of dust optical depth on Mars. Icarus 251, 65-95 (2015). Dynamic Mars. doi:10.1016/j.icarus.2014.12.034

J.E. Moores, M.T. Lemmon, H. Kahanpää, S.C.R. Rafkin, R. Francis, J. Pla-Garcia, K. Bean, R. Haberle, C. Newman, M. Mischna, A.R. Vasavada, M. de la Torre Juárez, N. Renno, J. Bell, F. Calef, B. Cantor, T.H. Mcconnochie, A.-M. Harri, M. Genzer, M.H. Wong, M.D. Smith, F.J. Martín-Torres, M.-P. Zorzano, O. Kemppinen, E. McCullough, Observational evidence of a suppressed planetary boundary layer in northern Gale Crater, Mars as seen by the Navcam instrument onboard the Mars Science Laboratory rover. Icarus 249(15), 129-142 (2015). doi:10.1016/j.icarus.2014.09.020

D.P. Mulholland, P.L. Read, S.R. Lewis, Simulating the interannual variability of major dust storms on Mars using variable lifting thresholds. Icarus 223(1), 344-358 (2013). doi:10.1016/j.icarus.2012.12.003

J.R. Murphy, S. Nelli, Mars Pathfinder convective vortices: Frequency of occurrence. Geophys. Res. Lett. 29(23), 18-1184 (2002). doi:10.1029/2002GL015214. http://dx.doi.org/10.1029/2002GL015214

L.D.V. Neakrase, R. Greeley, Dust devils in the laboratory: Effects of surface roughness on vortex dynamics. J. Geophys. Res. 115(E05003) (2010a). doi:10.1029/2009JE003465

L.D.V. Neakrase, R. Greeley, Dust devil sediment flux on Earth and Mars: Laboratory simulations. Icarus 206(1), 306-318 (2010b)

L.D.V. Neakrase, R. Greeley, J.D. Iversen, M.R. Balme, E.E. Eddlemon, Dust flux within dust devils: Preliminary laboratory simulations 33(L19S09), 2-6 (2006). doi:10.1029/2006GL026810 
C.E. Newman, S.R. Lewis, P.L. Read, The atmospheric circulation and dust activity in different orbital epochs on Mars. Icarus 174(1), 135-160 (2005). doi:10.1016/j.icarus.2004.10.023

C.E. Newman, M.I. Richardson, The impact of surface dust source exhaustion on the martian dust cycle, dust storms and interannual variability, as simulated by the MarsWRF General Circulation Model. Icarus 257, 47-87 (2015). doi:10.1016/j.icarus.2015.03.030

C.E. Newman, S.R. Lewis, P.L. Read, F. Forget, Modeling the Martian dust cycle, 1. Representations of dust transport processes. Journal of Geophysical Research: Planets 107(E12), 6-1618 (2002a). 5123. doi:10.1029/2002JE001910. http://dx.doi.org/10.1029/2002JE001910

C.E. Newman, S.R. Lewis, P.L. Read, F. Forget, Modeling the Martian dust cycle 2. Multiannual radiatively active dust transport simulations. Journal of Geophysical Research: Planets 107(E12), 7-1715 (2002b). 5124. doi:10.1029/2002JE001920. http://dx.doi.org/10.1029/2002JE001920

H. Ohno, T. Takemi, Mechanisms for intensification and maintenance of numerically simulated dust devils. Atmos. Sci. Let. (2010). doi:10.1002/asl

A.M.C. Oke, D. Dunkerley, N.J. Tapper, Willy-willies in the Australian landscape: Sediment transport characteristics. J. Arid Environ. 71(2), 216-228 (2007a). doi:10.1016/j.jaridenv.2007.03.014

A. Oke, N. Tapper, D. Dunkerley, Willy-willies in the Australian landscape: the role of key meteorological variables and surface conditions in defining frequency and spatial characteristics. J. Arid Environ. 71(2), 201-215 (2007b)

F. Pantillon, P. Knippertz, J.H. Marsham, C.E. Birch, A parameterization of convective dust storms for models with mass-flux convection schemes. J. Atmos. Sci. 72, 25452561 (2015). doi:10.1175/JAS-D-14-0341.1

A. Petrosyan, B. Galperin, S.E. Larsen, S.R. Lewis, A. Määttänen, P.L. Read, N. Renno, L.P.H.T. Rogberg, H. Savijärvi, T. Siili, A. Spiga, A. Toigo, L. Vázquez, The martian atmospheric boundary layer. Rev. Geophys. 49(3) (2011). doi:10.1029/2010RG000351

S. Raasch, T. Franke, Structure and formation of dust devil-like vortices in the atmospheric boundary layer: A high-resolution numerical study. J. Geophys. Res. 116(D16120), 1-16 (2011). doi:10.1029/2011JD016010

S.C.R. Rafkin, R.M. Haberle, T.I. Michaels, The Mars Regional Atmospheric Modeling System: Model Description and Selected Simulations. Icarus 151, 228-256 (2001)

P.L. Read, S.R. Lewis, The Martian climate revisited: atmosphere and environment of a desert planet (Springer, Berlin; New York, 2004)

D. Reiss, N.M. Hoekzema, O.J. Stenzel, Dust deflation by dust devils on Mars derived from optical depth measurements using the shadow method in HiRISE images. Planet. Space Sci. 93-94, 54-64 (2014). doi:10.1016/j.pss.2014.01.016

D. Reiss, J. Raack, H. Hiesinger, Bright dust devil tracks on Earth: Implications for their formation on Mars. Icarus 211(1), 917-920 (2011). doi:10.1016/j.icarus.2010.09.009

D. Reiss, A. Spiga, G. Erkerling, The horizontal motion of dust devils on Mars derived from CRISM and CTX/HiRISE observations. Icarus 227, 8-20 (2014). doi:10.1016/j.icarus.2013.08.028

D. Reiss, M. Zanetti, G. Neukum, Multitemporal observations of identical active dust devils 
on Mars with the High Resolution Stereo Camera (HRSC) and Mars Orbiter Camera (MOC). Icarus 215 (2011). doi:10.1016/j.icarus.2011.06.011

D. Reiss, J. Raack, A.P. Rossi, G. Di Achille, H. Hiesinger, First in-situ analysis of dust devil tracks on Earth and their comparison with tracks on Mars. Geophys. Res. Lett. 37(14) (2010). L14203. doi:10.1029/2010GL044016

D. Reiss, R.D. Lorenz, Dust Devil Track Survey at Elysium Planitia, Mars: Implications for the InSight landing sites. Icarus 266, 315-330 (2015). doi:doi:10.1016/j.icarus.2015.11.012

D. Reiss, M.I. Zimmerman, D.C. Lewellen, Formation of cycloidal dust devil tracks by redeposition of coarse sands in southern Peru: Implications for Mars. Earth Planet. Sci. Lett. 383, 7-15 (2013)

N.O. Renno, A.P. Ingersoll, Natural convection as a heat engine: A theory for CAPE. J. Atmos. Sci. 53, 572-585 (1996). doi:10.1175/15200469(1996)053;0572:NCAAHE ¿2.0.CO;2

N.O. Renno, M.L. Burkett, M.P. Larkin, A simple thermodynamical theory for dust devils. J. Atmos. Sci. 55, $3244-3252$ (1998). doi:10.1175/15200469(1998)055<3244:ASTTFD > 2.0.CO;2

N.O. Renno, V.J. Abreu, J. Koch, P.H. Smith, O.K. Hartogensis, H.A.R.D. Bruin, D. Burose, G.T. Delory, W.M. Farrell, C.J. Watts, J. Garatuza, M. Parker, A. Carswell, MATADOR 2002: A pilot field experiment on convective plumes and dust devils. J. Geophys. Res. 109 (2004). doi:10.1029/2003JE002219

N.O. Renno, A.A. Nush, J. Luninne, J. Murphy, Martian and terrestrial dust devils: Test of a scaling theory using Pathfinder data. J. Geophys. Res. 105, 1859-1866 (2000). doi:10.1029/19999JE001037

D. Rosenfeld, Y. Rudich, R. Lahav, Desert dust suppressing precipitation: A possible desertification feedback loop. Proc. Natl. Acad. Sci. USA 98(11), 5975-5980 (2001). doi:10.1073/pnas.101122798

J.A. Ryan, R.D. Lucich, Possible dust devils, vortices on mars. J. Geophys. Res. 88(C15), 11005-11011 (1983). doi:10.1029/JC088iC15p11005

J. Ryan, Relation of dust devil frequency and diameter to atmospheric temperature. J. Geophys. Res. 77(36), 7133-7137 (1972)

K. Schepanski, I. Tegen, M. Todd, B. Heinold, G. Bönisch, Meteorological processes forcing Saharan dust emission inferred from MSG-SEVIRI observations of subdaily dust source activation and numerical models. J. Geophys. Res. 114, 10201 (2012)

J.T. Schofield, J.R. Barnes, D. Crisp, R.M. Haberle, S. Larsen, J.A. Magalhaes, J.R. Murphy, A. Seiff, G. Wilson, The Mars Pathfinder atmospheric structure investigation meteorology (ASI/MET) experiment. Science 278(5344), 1752-1758 (1997). doi:10.1126/science.278.5344.1752

Y. Shao, Simplification of a dust emission scheme and comparison with data. J. Geophys. Res. 109 (2004). doi:10.1029/2003JD004372

Y. Shao, Physics and Modelling of Wind Erosion, 2nd edn. (Springer, Berlin, 2008), p. 452

Y. Shao, M. Klose, A note on the stochastic nature of particle cohesive force and implications to threshold friction velocity for aerodynamic dust entrainment. Aeolian Research (2016). submitted 
Y. Shao, H. Lu, A simple expression for wind erosion threshold friction velocity. J. Geophys. Res. 105, 22437-22443 (2000)

Y. Shao, M. Klose, K.-H. Wyrwoll, Recent global dust trend and connections to climate forcing. J. Geophys. Res. Atmos. 118, 1-12 (2013). doi:10.1002/jgrd.50836

Y. Shao, M.R. Raupach, P.A. Findlater, The effect of saltation bombardment on the entrainment of dust by wind. J. Geophys. Res. 98, 12719-12726 (1993)

Y. Shao, K.-H. Wyrwoll, A. Chappell, J. Huang, Z. Lin, G.H. McTainsh, M. Mikami, T.Y. Tanaka, X. Wang, S. Yoon, Dust cycle: An emerging core theme in Earth system science. Aeolian Research 2, 181-204 (2011). doi:10.1016/j.aeolia.2011.02.001

Y. Shao, S. Liu, J. Schween, S. Crewell, Large-eddy atmosphere-land-surface modelling over heterogeneous surfaces: Model development and comparison with measurements. Boundary-Layer Meteorol. 148(2), 333-356 (2013). doi:10.1007/s10546-013-9823-0

E.A. Shinn, G.W. Smith, J.M. Prospero, P. Betzer, M.L. Hayes, V. Garrison, R.T. Barber, African dust and the demise of Carribbean coral reefs. Geophys. Res. Lett. 27(19), 3029-3032 (2000). doi:10.1029/2000GL011599

P.C. Sinclair, General characteristics of dust devils. J. Appl. Meteor. 8, $32-45$ (1969). doi:10.1175/1520-0450(1969)008<0032:GCODD>2.0.CO;2

P.C. Sinclair, The lower structure of dust devils. J. Atmos. Sci. 30, 1599-1619 (1973). doi:10.1175/1520-0469(1973)0302.0.CO;2

M.D. Smith, Interannual variability in TES atmospheric observations of Mars during 1999-2003. Icarus 167(1), 148-165 (2004). Special Issue on DS1/Comet Borrelly. doi:10.1016/j.icarus.2003.09.010

J.T. Snow, T.M. McClelland, Dust devils at White Sands Missile Range, New Mexico: 1. Temporal and spatial distributions. J. Geophys. Res. Atmos. 95(D9), 13707-13721 (1990). doi:10.1029/JD095iD09p13707

I.N. Sokolik, O.B. Toon, Direct radiative forcing by anthropogenic airborne mineral aerosols. Nature 381, 681-683 (1996)

A. Spiga, F. Forget, A new model to simulate the Martian mesoscale and microscale atmospheric circulation: Validation and first results. J. Geophys. Res. 114, 02009 (2009). doi:10.1029/2008JE003242

A. Spiga, J. Faure, J.-B. Madeleine, A. Määttänen, F. Forget, Rocket dust storms and detached dust layers in the Martian atmosphere. J. Geophys. Res. 118(4), 746-767 (2013). doi:10.1002/jgre.20046

K.E. Steakley, J.R. Murphy, A year of convective vortex activity at Gale Crater (2016). accepted. doi:10.1016/j.icarus.2016.06.010

G. Sterk, L. Herrmann, A. Bationo, Wind-blown nutrient transport and soil productivity changes in southwest Niger. Land Degradation \& Development 7(4), 325-335 (1996). doi:10.1002/(SICI)1099-145X(199612)7:4¡325::AID-LDR237¡3.0.CO;2-Q

P.P. Sullivan, J.C. McWilliams, C.-H. Moeng, A subgrid-scale model for large-eddy simulation of planetary boundary-layer flows. Boundary-Layer Meteorol. 71(3), 247-276 (1994). doi:10.1007/BF00713741

T. Takemi, M. Yasui, J. Zhou, L. Liu, Role of boundary layer and cumulus convection on dust emission and transport over a midlatitude desert area. Journal of Geophysical Research: Atmospheres 111(D11) (2006). D11203. doi:10.1029/2005JD006666. http: 
//dx.doi.org/10.1029/2005JD006666

T.Y. Tanaka, M. Chiba, A numerical study of the contributions of dust source regions to the global dust budget. Global Planet. Change 52, 88-104 (2006)

P. Thomas, P.J. Gierasch, Dust devils on Mars. Science 230(4722), 175-177 (1985). doi:10.1126/science.230.4722.175

A.D. Toigo, M.I. Richardson, Meteorology of proposed Mars Exploration Rover landing sites. J. Geophys. Res. 108(E12), 8092 (2003). doi:10.1029/2003JE002064

C.A. Verba, P.E. Geissler, T.N. Titus, D. Waller, Observations from the High Resolution Imaging Science Experiment (HiRISE): Martian dust devils in Gusev and Russell craters. J. Geophys. Res. 115(E9) (2010). E09002. doi:10.1029/2009JE003498. http://dx.doi.org/10.1029/2009JE003498

H. Wang, M.I. Richardson, The origin, evolution, and trajectory of large dust storms on Mars during Mars years 2430 (1999-2011). Icarus 251, 112-127 (2015). Dynamic Mars. doi:10.1016/j.icarus.2013.10.033

N.P. Webb, C.L. Strong, A. Chappell, S.K. Marx, G.H. McTainsh, Soil organic carbon enrichment of dust emissions: magnitude, mechanisms and its implications for the carbon cycle. Earth Surf. Proc. Landforms 38(14), 1662-1671 (2013). doi:10.1002/esp.3404. http://dx.doi.org/10.1002/esp.3404

P.L. Whelley, R. Greeley, Latitudinal dependency in dust devil activity on Mars. Journal of Geophysical Research: Planets 111(E10) (2006). E10003. doi:10.1029/2006JE002677

P.L. Whelley, R. Greeley, The distribution of dust devil activity on Mars. J. Geophys. Res. 113(E7) (2008). doi:10.1029/2007JE002966. http://dx.doi.org/10.1029/ 2007 JE002966

G. Wurm, J. Teiser, D. Reiss, Greenhouse and thermophoretic effects in dust layers: The missing link for lifting of dust on Mars. Geophys. Res. Lett. 35(L10201) (2008). doi:doi:10.1029/2008GL033799

Y.Z. Zhao, Z.L. Gu, Y.Z. Yu, Y. Ge, Y. Li, X. Feng, Mechanism and large eddy simulation of dust devils. Atmosphere-Ocean 42(1), 61-84 (2004). doi:10.3137/ao.420105

A.D. Zimon, Adhesion of dust and powder (Consultants Bureau, New York, 1982), p. 438 